\title{
TRIHEDRAL SOERGEL BIMODULES
}

\author{
MARCO MACKAAY, VOLODYMYR MAZORCHUK, \\ VANESSA MIEMIETZ AND DANIEL TUBBENHAUER
}

\begin{abstract}
The quantum Satake correspondence relates dihedral Soergel bimodules to the semisimple quotient of the quantum $\mathfrak{s l}_{2}$ representation category. It also establishes a precise relation between the simple transitive 2-representations of both monoidal categories, which are indexed by bicolored ADE Dynkin diagrams.

Using the quantum Satake correspondence between affine $A_{2}$ Soergel bimodules and the semisimple quotient of the quantum $\mathfrak{s l}_{3}$ representation category, we introduce trihedral Hecke algebras and Soergel bimodules, generalizing dihedral Hecke algebras and Soergel bimodules. These have their own Kazhdan-Lusztig combinatorics and simple transitive 2-representations corresponding to tricolored generalized ADE Dynkin diagrams.
\end{abstract}

\section{CONTENTS}

1. Introduction

2. Some $\mathfrak{s l}_{3}$ combinatorics $\quad 6$

2A. Quantum $\mathfrak{s l}_{3} \quad 6$

2B. Chebyshev-like polynomials for $\mathfrak{s l}_{3} \quad 8$

3. Trihedral Hecke algebras 11

3A. Some color conventions 11

3B. The trihedral Hecke algebra of level $\infty \quad 12$

3C. The quotient of level $e \quad 15$

3D. Generalizing dihedral Hecke algebras 20

4. Trihedral Soergel bimodules 21

4A. Bott-Samelson bimodules for affine $\mathrm{A}_{2} \quad 21$

4B. The trihedral Soergel bimodules of level $\infty \quad 27$

4C. The 2-quotient of level $e \quad 29$

4D. Generalizing dihedral Soergel bimodules 33

5. Trihedral 2-representation theory 34

5A. Decategorified story 38

5B. Categorified story 45

5C. Trihedral zig-zag algebras 50

5D. Generalizing dihedral 2-representation theory 54

Appendix: Generalized ADE Dynkin diagrams $\quad 54$

$\begin{array}{ll}\text { References } & 57\end{array}$

\section{INTRODUCTION}

Non-negative integral representation theory. In pioneering work [KL79], KazhdanLusztig defined their celebrated bases of Hecke algebras for Coxeter groups. Crucially, on these bases the structure constants of the algebras belong to $\mathbb{N}^{0}=\mathbb{Z}_{>0}$. This started a program to study $\mathbb{N}^{0}$-algebras, which have a fixed basis with non-negative integral structure constants, see e.g. [Lus87], [EK95], where these algebras are called $\mathbb{Z}_{+}$-rings. 
As proposed by the work of Kazhdan-Lusztig, for $\mathbb{N}^{0}$-algebras it makes sense to study and classify $\mathbb{N}^{0}$-representations, i.e. representations with a fixed basis on which the fixed bases elements of the algebra act by non-negative integral matrices, see e.g. [EK95]. The first examples are the so-called cell representations, which were originally defined for Hecke algebras [KL79], but can be defined for all $\mathbb{N}^{0}$-algebras (and even $\mathbb{R}_{\geq 0}$-algebras, see [KM16]). As it turns out, $\mathbb{N}^{0}$-representations are interesting from various points of view, with applications and connections to e.g. graph theory, conformal field theory, modular tensor categories and subfactor theory.

Categorical analogs of $\mathbb{N}^{0}$-algebras are monoidal categories, which we consider as one-object 2 -categories, or 2-categories. These decategorify to $\mathbb{N}^{0}$-algebras, because the isomorphism classes of the indecomposable 1-morphisms form naturally a $\mathbb{N}^{0}$-basis. For example, Hecke algebras of Coxeter groups are categorified by Soergel bimodules [Soe92] such that indecomposable bimodules decategorify to the Kazhdan-Lusztig basis elements [EW16].

The categorical incarnation of $\mathbb{N}^{0}$-representation theory is 2-representation theory. Any 2-representation decategorifies naturally to a $\mathbb{N}^{0}$-representation, with the $\mathbb{N}^{0}$-basis given by the isomorphism classes of the indecomposable 1-morphisms. However, not all $\mathbb{N}^{0}$-representations can be obtained in this way.

In 2-representation theory, the simple transitive 2-representations play the role of the simple representations [MM16b]. Although their decategorifications need not be simple as complex representations, they are the "simplest" 2-representations, as attested e.g. by the categorical Jordan-Hölder theorem [MM16b]. This naturally motivates the problem of classification of simple transitive 2-representations of 2-categories. Just as the cell representations form a natural class of $\mathbb{N}^{0}$-representations of any $\mathbb{N}^{0}$-algebra, cell 2-representations form a natural class of simple transitive 2-representations of any finitary 2-category (i.e. 2-categories with certain finiteness conditions [MM11]). A crucial difference is that cell 2-representations are always simple transitive, while cell representations are usually not simple.

In this paper, we restrict our attention to certain subquotients of the Hecke algebra of affine type $\mathrm{A}_{2}$, which we call trihedral Hecke algebras, and their categorification by subquotients of Soergel bimodules of affine type $\mathrm{A}_{2}$, which we call trihedral Soergel bimodules. These should have 2-representations indexed by tricolored generalized ADE Dynkin diagrams with trihedral zig-zag algebras making their appearance. As we explain below, we think of these as rank three analogues of dihedral Hecke algebras, dihedral Soergel bimodules and zig-zag algebras.

The dihedral story. For finite Coxeter types, the classification of the simple transitive 2-representations of Soergel bimodules is only partially known, see e.g. [KMMZ16], [MMMZ18], [Zim17]. There are two exceptions: For Coxeter type A, the cell 2-representations exhaust the simple transitive 2-representations of Soergel bimodules [MM16b], so the classification problem has been solved. For Coxeter type $\mathrm{I}_{2}(e+2)$, which is the type of the dihedral group with $2(e+2)$ elements, there also exists a complete classification of simple transitive 2-representations [KMMZ16], [MT16] (for $e=10,16$ or 28 the classification is only known under the additional assumption of gradeability), which is completely different from the one for type A. In this case, the simple transitive 2-representations of rank greater than one are classified by bicolored ADE Dynkin diagrams, with the cell 2-representations being the ones corresponding to Dynkin diagrams of type A. The others, corresponding to Dynkin types $\mathrm{D}$ and $\mathrm{E}$, are not equivalent to cell 2-representations and revealed interesting new features in 2-representation theory, e.g. the two bicolorings of type $E_{7}$ give non-equivalent 2-representations which categorify the same $\mathbb{N}^{0}$-representation [MT16]. For completeness, we remark that there are precisely two rank-one 2-representations corresponding to the highest and the lowest two-sided cells, which categorify the trivial and the sign representation of the Hecke algebra. We note that going to the small quotient $\mathscr{D}_{e}$ by annihilating the highest cell avoids that we have to worry about the categorical analog of the trivial representation. 
This case is particularly interesting because of Elias' quantum Satake correspondence [Eli16], [Eli17] between $\mathcal{Q}_{e}\left(\mathfrak{s l}_{2}\right)$ and $\mathscr{D}_{e}$. Here $\mathcal{Q}_{e}\left(\mathfrak{s l}_{2}\right)$ denotes the semisimple quotient of the monoidal category of finite-dimensional quantum $\mathfrak{s l}_{2}$-modules (of type 1 ), where the quantum parameter $\eta$ is a primitive, complex $2(e+2)^{\text {th }}$ root of unity. This correspondence is given by a nice, but slightly technical 2-functor, so we omit further details at this stage.

Note that, when $\mathrm{q}$ is generic, the quantum Satake correspondence also exists, but between the whole category of finite-dimensional quantum $\mathfrak{s l}_{2}$-modules $\mathcal{Q}_{\mathbf{q}}\left(\mathfrak{s l}_{2}\right)$ and Soergel bimodules of the infinite dihedral type $\mathrm{I}_{2}(\infty)$, which coincides with affine type $A_{1}$.

One consequence of Elias' Satake correspondence is a precise relation between the simple transitive 2-representations of $\mathcal{Q}_{e}\left(\mathfrak{s l}_{2}\right)$ and $\mathscr{D}_{e}$. However, the corresponding 2-representations are not equivalent, because $\mathcal{Q}_{e}\left(\mathfrak{s l}_{2}\right)$ is semisimple while $\mathscr{D}_{e}$ is not.

Let us explain this in a bit more detail. Equivalence classes of simple transitive 2-representations of finitary 2-categories (or graded versions of them) correspond bijectively to Morita equivalence classes of simple algebra 1-morphisms in the abelianizations of these 2-categories. This was initially proved for semisimple tensor categories [Ost03] and later generalized to certain finitary 2-categories with duality [MMMT16]. Kirillov-Ostrik [KJO02] classified the simple algebra 1-morphisms in $\mathcal{Q}_{e}\left(\mathfrak{s l}_{2}\right)$ up to Morita equivalence, under some natural assumptions, in terms of ADE Dynkin diagrams. From their results, via the quantum Satake correspondence, we can get all indecomposable algebra 1-morphisms, up to Morita equivalence, in $\mathscr{D}_{e}$. (The latter is additive but not abelian, which is why we get indecomposable instead of simple algebra 1-morphisms.)

Given an ADE Dynkin diagram $\boldsymbol{\Gamma}$ and the corresponding algebra 1-morphism $\mathrm{A}^{\boldsymbol{\Gamma}}$, the category underlying the 2-representation of $\mathcal{Q}_{e}\left(\mathfrak{s l}_{2}\right)$ is equivalent to the category of $\mathrm{A}^{\boldsymbol{\Gamma}}$-modules in $\mathcal{Q}_{e}\left(\mathfrak{s l}_{2}\right)$. The quiver of this category is trivial: its vertices coincide with those of $\boldsymbol{\Gamma}$, but it has no edges because $\mathcal{Q}_{e}\left(\mathfrak{s l}_{2}\right)$ is semisimple. However, the quiver underlying the corresponding simple transitive 2-representation of $\mathscr{D}_{e}$ is the so-called doubled quiver of type $\boldsymbol{\Gamma}$, which has two oppositely oriented edges between each pair of adjacent vertices. Its quiver algebra, the zig-zag algebra, was for example studied by Huerfano-Khovanov [HK01]. It has very nice properties and shows up in various mathematical contexts nowadays.

Kirillov-Ostrik's classification can be seen as a quantum version of the McKay correspondence between finite subgroups of SU(2) and ADE Dynkin diagrams. The vertices of such a Dynkin diagram $\boldsymbol{\Gamma}$ correspond to the simple $\mathrm{A}^{\boldsymbol{\Gamma}}$-modules in $\mathcal{Q}_{e}\left(\mathfrak{s l}_{2}\right)$. These module categories decategorify to $\mathbb{N}^{0}$-representations of the Grothendieck group of $\mathcal{Q}_{e}\left(\mathfrak{s l}_{2}\right)$, the so-called Verlinde algebra, which were classified by Etingof-Khovanov [EK95]. The Verlinde algebra is isomorphic to a polynomial algebra in one variable quotient by the ideal generated by the $(e+1)^{\text {th }}$ Chebyshev polynomial $\mathrm{U}_{e+1}(\mathrm{x})$ (normalized and of the second kind). Thus, Etingof-Khovanov basically classified all non-negative integer matrices which are killed by $\mathrm{U}_{e+1}(\mathrm{X})$. (Note that not all of them come from 2-representations of $\mathcal{Q}_{e}\left(\mathfrak{s l}_{2}\right)$, because some correspond to graphs which are not Dynkin diagrams of type ADE.)

Similarly, the Hecke algebra $\mathrm{H}_{\mathrm{v}}\left(\mathrm{I}_{2}(e+2)\right)$ of Coxeter type $\mathrm{I}_{2}(e+2)$ can be obtained as a quotient of the Hecke algebra $H_{v}\left(\tilde{A}_{1}\right)$ of affine type $A_{1}$, where $v$ is a generic parameter (the decategorification of the grading within the Soergel 2-category). Let $\theta_{s}, \theta_{t}$ denote the Kazhdan-Lusztig generators corresponding to the simple reflections, in both $\mathrm{H}_{\mathrm{v}}\left(\mathrm{I}_{2}(e+2)\right)$ and $\mathrm{H}_{\mathrm{v}}\left(\tilde{\mathrm{A}}_{1}\right)$. Furthermore, let $\theta_{w_{0}}$ be the Kazhdan-Lusztig basis element in $\mathrm{H}_{\mathrm{v}}\left(\mathrm{l}_{2}(e+2)\right)$ for the longest word in the dihedral group. Then there are two ways to write $\theta_{w_{0}}$ as a linear combination of alternating products of $\theta_{s}$ and $\theta_{t}$, which only differ by the choice of the fixed final Kazhdan-Lusztig generator in each product. The coefficients in both linear combinations are precisely the coefficients of $\mathrm{U}_{e+1}(\mathrm{X})$. (This observation is implicit in [Lus83].) Then $\mathrm{H}_{\mathrm{v}}\left(\mathrm{I}_{2}(e+2)\right)$ is obtained from $\mathrm{H}_{\mathrm{v}}\left(\tilde{\mathrm{A}}_{1}\right)$ by declaring both these linear combinations to be equal to each other. By declaring them to be equal to zero, we obtain the small quotient $\mathrm{D}_{e}$ of 
$\mathrm{H}_{\mathrm{v}}\left(\mathrm{I}_{2}(e+2)\right)$, which is precisely the algebra that corresponds to the Verlinde algebra under the quantum Satake correspondence. Moreover, one can show that these algebras have a very similar $\mathbb{N}^{0}$-representation theory.

To conclude, one could say that Elias' quantum Satake correspondence [Eli16], [Eli17] categorifies the relation between the Verlinde algebra and the small dihedral quotient, while the results from [KMMZ16], [MT16], [MMMT16] categorify the relations between their $\mathbb{N}^{0}$-representations.

The trihedral story. Now let us get to the topic of this paper. Elias also defined a quantum Satake correspondence between $\mathcal{Q}_{e}=\mathcal{Q}_{e}\left(\mathfrak{s l}_{3}\right)$ and the 2-category of Soergel bimodules of affine type $A_{2}$ [Eli17]. In this paper, we study certain subquotients of these Soergel bimodules, depending on a choice of a primitive, complex $2(e+3)^{\text {th }}$ root of unity $\eta$, and their 2representation theory. Our construction uses the quantum Satake correspondence with $\mathcal{Q}_{e}$, whose Grothendieck group is isomorphic to a polynomial algebra in two variables quotient by the ideal generated by a set of polynomials $\mathrm{U}_{m, n}(\mathrm{X}, \mathrm{Y})$, for $m+n=e+1, m, n \in \mathbb{N}^{0}$. These polynomials were introduced to the field of orthogonal polynomials by Koornwinder [Koo74] and they generalize the Chebyshev polynomials. To the best of our knowledge, these subquotients are new and have not been studied before.

In fact, even their decategorifications, which are certain subquotients of the Hecke algebra $\mathrm{H}_{\mathrm{v}}\left(\tilde{\mathrm{A}}_{2}\right)$ of affine type $\mathrm{A}_{2}$, seem to be new. For each $e \in \mathbb{N}^{0}$, we call the corresponding subquotient the trihedral Hecke algebra of level $e$ and denoted it by $\mathrm{T}_{e}$. These algebras have their own Kazhdan-Lusztig combinatorics and interesting $\mathbb{N}^{0}$-representations. We see the trihedral Hecke algebras as rank three analogues of the small quotients of the dihedral Hecke algebras. There are many similarities, but also some differences. For example, as far as we can tell, the trihedral Hecke algebras are not deformations of any group algebra. But they are semisimple algebras and the classification of their irreducible representations runs in parallel to the analogous classification for dihedral Hecke algebras, and their $\mathbb{N}^{0}$-representation theory has also a very similar behavior.

Now to the categorified story: In the trihedral case, the quantum Satake correspondence for q being generic only gives a 2-subcategory of the affine type $\mathrm{A}_{2}$ Soergel 2-category. We call this the 2-category of trihedral Soergel bimodules of level $\infty$ and denote it by $\mathscr{T}_{\infty}$. The 2-category $\mathscr{T}_{\infty}$ admits quotients $\mathscr{T}_{e}$, the trihedral Soergel bimodules of level $e$, which via the quantum Satake correspondence for $\eta$ is related to $\mathcal{Q}_{e}$. The corresponding decategorifications are the trihedral Hecke algebras $\mathrm{T}_{\infty}$ and $\mathrm{T}_{e}$.

Coming back to representation theory, people have studied the $\mathbb{N}^{0}$-representations of the Grothendieck group of $\mathcal{Q}_{e}$, as they arise in conformal field theory and the study of fusion categories/modular tensor categories, see e.g. [Gan94], [EP10], [Sch17] and related works. This time, four families of graphs play an important role and, by analogy with the $\mathfrak{s l}_{2}$ case, their types are called A, conjugate A, D and E, although they are not Dynkin diagrams. Their adjacency matrices, which are non-negative integral matrices, are annihilated by Koornwinder's polynomials, just as in [EK95]. Furthermore, the type A graphs can be seen as a cut-off of the positive Weyl chamber of $\mathfrak{s l}_{3}$, just as the usual type $A$ Dynkin diagrams can be seen as cut-offs for $\mathfrak{s l}_{2}$. Finally, the type $\mathrm{D}$ graphs for $\mathfrak{s l}_{3}$ come from a $\mathbb{Z} / 3 \mathbb{Z}$-symmetry of these cut-offs, just as the type $D$ Dynkin diagrams come from a $\mathbb{Z} / 2 \mathbb{Z}$-symmetry.

Simple algebra 1-morphisms in $\mathcal{Q}_{e}$ and the corresponding simple transitive 2-representations have also been studied e.g. in [Sch17] and are closely related to these ADE type graphs. Via the quantum Satake correspondence, we therefore get indecomposable algebra 1-morphisms in $\mathrm{T}_{e}$ and the corresponding simple transitive 2-representations of the trihedral Soergel bimodules. Since we are not familiar with some of the ingredients in the construction of algebra 1morphisms in [Sch17], we have given an alternative construction, using the symmetric $\mathfrak{s l}_{3}$-web 
calculus, as in [RT16], [TVW17]. For this reason, our construction so far only works for types $A$ and $D$, so we restrict our attention to those two types. Almost by construction, the cell 2-representations are equivalent to the simple transitive 2-representations of type A. The ones of type $\mathrm{D}$ have a different rank and are therefore inequivalent. For the other types, we have no conjectures at all, and we are not even sure whether they correspond to 2-representations.

Computing the quiver algebras explicitly proved to be much harder this time. We define type A quiver algebras which, up to scalars, are the ones underlying the cell 2-representations of $\mathscr{T}_{e}$. These algebras are the trihedral analogs of the zig-zag algebras of type A, e.g. the endomorphisms algebras of their vertices are the cohomology rings of the full flag variety of flags in $\mathbb{C}^{3}$, instead of the flags in $\mathbb{C}^{2}$ as in the dihedral case. For this reason, we call them trihedral zig-zag algebras. The type $D$ trihedral zig-zag algebras can be obtained from these by using the $\mathbb{Z} / 3 \mathbb{Z}$-symmetry, just as the $\mathrm{D}$ dihedral zig-zag algebras can be obtained from the type $A$ via a $\mathbb{Z} / 2 \mathbb{Z}$-symmetry, but we have not worked out the details.

Finally, let us stress that our trihedral zig-zag algebras are different from Grant's [Gra17] higher zig-zag algebras, which are only subalgebras of the trihedral zig-zag algebras of type A, although both underlying graphs come from a cut-off of the positive Weyl chamber of $\mathfrak{s l}_{3}$.

The Nhedral story. We expect that our story generalizes to $\mathfrak{s l}_{N}$ for arbitrary $N \geq 2$ : the Soergel bimodules of affine type $\mathrm{A}_{N-1}$ are known, the quantum Satake correspondence is conjectured to exist, the analogues of Koornwinder's Chebyshev polynomials are also known, and the corresponding generalized ADE type graphs appear in the mathematical physics literature on fusion algebras or the classification of subgroups of quantum $\mathrm{SU}(N)$, see e.g. [DFZ90], [Ocn02]. We expect that there exist Nhedral algebras and Nhedral Soergel bimodules of level $e$ (where $\eta$ would be a primitive, complex $2(e+N)^{\text {th }}$ root of unity), and Nhedral zig-zag algebras of ADE-type quivers such that the endomorphism algebra of every vertex is isomorphic to the cohomology ring of the full flag variety of $\mathbb{C}^{N}$.

Remark about colors. We use colors in this paper, and the colors which we need are blue $\boldsymbol{\square}$, red $\boldsymbol{\square}$, yellow $\square$, green $\boldsymbol{\square}$, orange $\square$ and purple $\boldsymbol{\square}$ which will appear as indicated by the preceding boxes.

Quantum conventions. The notation $\mathrm{v}$ will mean a generic parameter which plays the role of the decategorification of the grading which we will me in Section 4.

In contrast, $q$ will also denote a generic parameter, but it will turn up on the categorified level as our quantum parameter. Moreover, $\eta$ will be a primitive, complex $2(e+3)^{\text {th }}$ root of unity $\eta^{2(e+3)}=1$ which is a specialization of q, but never of v. Here $e \in \mathbb{N}^{0}=\mathbb{Z}_{\geq 0}$ will usually be arbitrary, but fixed, and is called the level.

The ground field will always be $\mathbb{C}_{\mathrm{v}}=\mathbb{C}(\mathrm{v}), \mathbb{C}_{\mathrm{q}}=\mathbb{C}(\mathrm{q})$ or $\mathbb{C}=\mathbb{C}(\eta)$, if not stated otherwise. Sometimes, instead of working over a ground field, we will work over rings as e.g. $\mathbb{Z}_{[\mathrm{v}]}=\mathbb{Z}\left[\mathrm{v}, \mathrm{v}^{-1}\right]$ or semirings as e.g. $\mathbb{N}_{[\mathrm{v}]}^{0}=\mathbb{N}^{0}\left[\mathrm{v}, \mathrm{v}^{-1}\right]$ and their quantum counterparts. (It will be clear from the notation whether we work with v or q. Moreover, a subscript [_] will always indicate that we are in the case of (semi)rings rather than fields.) In this context, we use the v-numbers, factorials and binomials, where $s \in \mathbb{Z}, t \in \mathbb{Z}_{\geq 1}$

$$
[s]_{\mathrm{v}}=\frac{\mathrm{v}^{s}-\mathrm{v}^{-s}}{\mathrm{v}-\mathrm{v}^{-1}}, \quad[t]_{\mathrm{v}} !=[t]_{\mathrm{v}}[t-1]_{\mathrm{v}} \ldots[1]_{\mathrm{v}}, \quad\left[\begin{array}{l}
s \\
t
\end{array}\right]_{\mathrm{v}}=\frac{[s]_{\mathrm{v}}[s-1]_{\mathrm{v}} \ldots[s-t+1]_{\mathrm{v}}}{[t]_{\mathrm{v}}[t-1]_{\mathrm{v}} \ldots[1]_{\mathrm{v}}}
$$

all of which are in $\mathbb{Z}_{[\mathrm{v}]}$. By convention, $[0]_{\mathrm{v}} !=1=\left[\begin{array}{l}s \\ 0\end{array}\right]_{\mathrm{v}}$. Note that $[0]_{\mathrm{v}}=0=\left[\begin{array}{c}0 \leq s<t \\ t\end{array}\right]_{\mathrm{v}}$ and $[-s]_{\mathrm{v}}=-[s]_{\mathrm{v}}$. Similarly with $\mathrm{q}$ or $\eta$ instead of $\mathrm{v}$.

Acknowledgements. We thank Ben Elias for generously sharing his ideas and his insights into the field of Soergel bimodules. We also thank Rostyslav Kozhan for his help with 
orthogonal polynomials, and Joseph Grant and Mikhail Khovanov for some helpful discussions. D.T. likes to thank SAGE for disproving all of his conjectures related to this project.

A part of this paper was written while D.T. participated in the Junior Trimester Program "Symplectic Geometry and Representation Theory" of the Hausdorff Research Institute for mathematics (HIM). The hospitality of the HIM during this period is gratefully acknowledged.

M.M. was partially supported by FCT/Portugal through project UID/MAT/04459/2013, Vo.Ma. was supported by the Swedish Research Council and Göran Gustafssons Stiftelse during this project, and D.T. is grateful to NCCR SwissMAP for generous support.

\section{Some $\mathfrak{s l}_{3}$ COMBinatorics}

This section is mostly a collection of known results, formulated in our notation.

2A. Quantum $\mathfrak{s l}_{3}$. Throughout, $m, n, k, l$ will denote non-negative integers.

Some conventions. We always use the following conventions when working with $\mathfrak{s l}_{3}$. Denote by $\varepsilon_{1}, \varepsilon_{2}, \varepsilon_{3}$ the standard basis vectors of $\mathbb{R}^{3}$. We endow $\mathbb{R}^{3}$ with the usual symmetric bilinear form $\left(\varepsilon_{i}, \varepsilon_{j}\right)=\delta_{i j}$ and let $E=\left\{\left(x_{1}, x_{2}, x_{3}\right) \in \mathbb{R}^{3} \mid x_{1}+x_{2}+x_{3}=0\right\}$ be the Euclidean subspace of $\mathbb{R}^{3}$ (with induced symmetric bilinear form). We also fix two simple roots $\alpha_{1}=\varepsilon_{1}-\varepsilon_{2}$ and $\alpha_{2}=\varepsilon_{2}-\varepsilon_{3}$, and coroots $\alpha_{1}^{\vee}$ and $\alpha_{2}^{\vee}$ such that $\left\langle\alpha_{i}, \alpha_{j}^{\vee}\right\rangle=\left(\alpha_{i}, \alpha_{j}\right)=a_{i j}$, for $i, j=1,2$, are the entries of the (usual) Cartan matrix $\left(\begin{array}{cc}2 & -1 \\ -1 & 2\end{array}\right)$ of $\mathfrak{s l}_{3}$. The (integral) weights are $X=\left\{\lambda \in E \mid\left\langle\lambda, \alpha_{1}^{\vee}\right\rangle \in \mathbb{Z}\right.$ and $\left.\left\langle\lambda, \alpha_{2}^{\vee}\right\rangle \in \mathbb{Z}\right\}$. The dominant (integral) weights are $X^{+}=\left\{\lambda \in E \mid\left\langle\lambda, \alpha_{1}^{\vee}\right\rangle \in \mathbb{N}^{0}\right.$ and $\left.\left\langle\lambda, \alpha_{2}^{\vee}\right\rangle \in \mathbb{N}^{0}\right\}$. We identify $X=\mathbb{Z}^{2}$ and $X^{+}=\left(\mathbb{N}^{0}\right)^{2}$, cf. (2-1), with $X^{+}$also called the positive Weyl chamber.

The following picture summarizes our root and weight conventions:

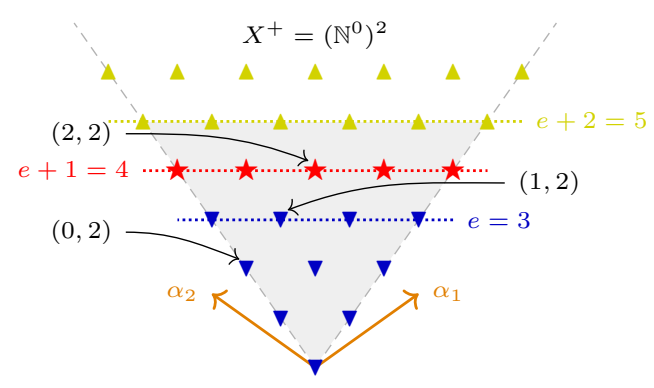

We have also indicated an example of a cut-off, denoting its weights by $\mathbf{v}$, which depend on the level $e$, i.e. the integral points $X^{+}(e)=\left\{\lambda \in X^{+} \mid\left\langle\lambda, \alpha_{1}^{\vee}\right\rangle \leq e\right.$ and $\left.\left\langle\lambda, \alpha_{2}^{\vee}\right\rangle \leq e\right\}$, and plays an important role in our paper. Moreover, we usually quotient by data associated to the line $e+1$ as illustrate by the symbols $\star$ in $(2-1)$.

Generic quantum $\mathfrak{s l}_{3}$. Let $\mathrm{U}_{\mathrm{q}}\left(\mathfrak{s l}_{3}\right)$ denote the quantum enveloping $\left(\mathbb{C}_{\mathrm{q}^{-}}\right)$algebra associated to $\mathfrak{s l}_{3}$. We refer the reader to [Jan96, Chapters 4-7] (whose conventions we silently adopt using the root and weight setting from above) for details. We denote by $\mathcal{Q}_{\mathrm{q}}=\mathrm{U}_{\mathrm{q}}\left(\mathfrak{s l}_{3}\right)-\mathcal{M}$ od the category of finite-dimensional (left) $\mathrm{U}_{\mathrm{q}}\left(\mathfrak{s l}_{3}\right)$-modules (of type 1, cf. [Jan96, Section 5.2]). Recall that $\mathcal{Q}_{\mathrm{q}}$ is semisimple with a complete set of pairwise non-isomorphic, irreducible $\mathrm{U}_{\mathrm{q}}\left(\mathfrak{s l}_{3}\right)$-modules parametrized by (the integral part of) the positive Weyl chamber

$$
\left\{\mathrm{L}_{m, n} \mid(m, n) \in X^{+}\right\} \text {. }
$$

The subscripts $m, n$ indicate the highest weight $m \varepsilon_{1}+n \varepsilon_{2}$ of the simple module, by which it is uniquely determined. Moreover, $\mathrm{U}_{\mathrm{q}}\left(\mathfrak{s l}_{3}\right)$ is a Hopf algebra, so we can tensor $\mathrm{U}_{\mathrm{q}}\left(\mathfrak{s l}_{3}\right)$-modules and take duals. Thus, if [_] $]_{\oplus}$ denotes the (additive) Grothendieck group, then

$$
\left\{\left[\mathrm{L}_{m, n}\right]=\left[\mathrm{L}_{m, n}\right]_{\oplus} \in\left[\mathcal{Q}_{\mathrm{q}}\right]_{\oplus} \mid(m, n) \in X^{+}\right\}
$$


is a $\mathbb{Z}$-basis of $\left[\mathcal{Q}_{\mathrm{q}}\right]_{\oplus}$, and $\left[\mathcal{Q}_{\mathrm{q}}\right]_{\oplus}$ is a ring. Extending the scalars to $\mathbb{C}$, we get a $\mathbb{C}$-algebra:

$$
\left[\mathcal{Q}_{\mathrm{q}}\right]_{\oplus}^{\mathbb{C}}=\left[\mathcal{Q}_{\mathrm{q}}\right]_{\oplus} \otimes_{\mathbb{Z}} \mathbb{C}
$$

Throughout the paper, we will use the notation $\left.\left[\_\right]\right]_{\oplus}^{\mathbb{C}}$.

Remark 2.1. Since $q$ is generic, we can identify $\left[\mathcal{Q}_{\mathrm{q}}\right]_{\oplus}$ with the corresponding Grothendieck ring of the category of complex, finite-dimensional representations of $\mathfrak{s l}_{3}$, cf. [Jan96, Theorems 5.15 and 5.17]. This means that all our calculations below follow from standard results in the representation theory of $\mathfrak{s l}_{3}$.

The two $\mathrm{U}_{\mathrm{q}}\left(\mathfrak{s l}_{3}\right)$-modules

$$
\mathrm{X}=\mathrm{L}_{1,0}, \quad \mathrm{Y}=\mathrm{L}_{0,1}
$$

are called the fundamental representations of $\mathfrak{s l}_{3}$. Note that they are dual, i.e. $\mathrm{X}^{*} \cong \mathrm{Y}$ as $\mathrm{U}_{\mathrm{q}}\left(\mathfrak{s l}_{3}\right)$-modules. More generally, we have $\left(\mathrm{L}_{m, n}\right)^{*} \cong \mathrm{L}_{n, m}$ for all $m, n \in \mathbb{N}^{0}$.

In the following we write $\mathrm{X}^{k}=\mathrm{X}^{\otimes k}, \mathrm{Y}^{l}=\mathrm{Y}^{\otimes l}$ and $\mathrm{XY}=\mathrm{X} \otimes \mathrm{Y}$ for short, and below we will consider these as variables in some polynomial ring.

Remark 2.2. Every $\mathrm{L}_{m, n}$ appears as a direct summand of a suitable tensor product of $\mathrm{X}$ and $\mathrm{Y}$. Moreover, $\mathcal{Q}_{\mathrm{q}}$ is braided monoidal, so $\mathrm{XY} \cong \mathrm{YX}$ as $\mathrm{U}_{\mathrm{q}}\left(\mathfrak{s l}_{3}\right)$-modules. Hence, the Grothendieck group of $\mathcal{Q}_{\mathrm{q}}$ is a commutative ring and

$$
\left\{\left[\mathrm{X}^{k} \mathrm{Y}^{l}\right] \mid(k, l) \in X^{+}\right\}
$$

is an alternative basis of $\left[\mathcal{Q}_{\mathbf{q}}\right]_{\oplus}$ and $\left[\mathcal{Q}_{\mathbf{q}}\right]_{\oplus}^{\mathbb{C}}$.

Using the above, in particular Remark 2.2 , we define $d_{m, n}^{k, l} \in \mathbb{Z}$ as follows:

$$
\left[\mathrm{L}_{m, n}\right]=\sum_{k, l} d_{m, n}^{k, l} \cdot\left[\mathrm{X}^{k} \mathrm{Y}^{l}\right]
$$

Clearly, $d_{m, n}^{k, l}=0$ unless $m+n \leq k+l$. Thus, the sum in (2-3) is finite.

Note that the $d_{m, n}^{k, l}$ can be computed inductively, cf. Example 2.6. Moreover, we have $d_{m, n}^{k, l}=d_{n, m}^{l, k}$ and $d_{m, n}^{m, n}=1=d_{k, l}^{k, l}$.

Definition 2.3. For later usage, let us define colors associated to the L's:

$$
\chi_{c}\left(\mathrm{~L}_{m, n}\right)= \begin{cases}g, & \text { if } m+2 n \equiv 0 \bmod 3, \\ o, & \text { if } m+2 n \equiv 1 \bmod 3, \\ p, & \text { if } m+2 n \equiv 2 \bmod 3 .\end{cases}
$$

We call $\chi_{c}\left(\mathrm{~L}_{m, n}\right)$ the central character of $\mathrm{L}_{m, n}$.

(To explain our choice of name: The center of $\mathrm{SU}_{3}$ is $\mathbb{Z} / 3 \mathbb{Z}$. The generator of $\mathbb{Z} / 3 \mathbb{Z}$ can act on any irreducible $\mathrm{SU}_{3}$-module by multiplication with a primitive, complex, third root of unity. This is what is encoded by $\chi_{c}$.)

Observe that $\chi_{c}(\mathrm{X})=o$ and $\chi_{c}(\mathrm{Y})=p$, while the representation theory of $\mathfrak{s l}_{3}$ immediately gives that tensoring with $\mathrm{X}$ changes the central character by adding $1 \bmod 3$, while tensoring with $Y$ adds $2 \bmod 3$. Thus:

Lemma 2.4. All irreducible summands of $\mathrm{X}^{k} \mathrm{Y}^{l}$ have central character $\chi_{c}\left(\mathrm{~L}_{k, l}\right)$. 
The semisimplified root of unity case. Let $\mathrm{U}_{\eta}\left(\mathfrak{s l}_{3}\right)$ be the specialization of (the integral form of) $\mathrm{U}_{\mathrm{q}}\left(\mathfrak{s l}_{3}\right)$ obtained by specializing q to $\eta$, see e.g. [Lus90], [APW91] for details.

Its category $\mathcal{Q}_{\eta}=\mathrm{U}_{\eta}\left(\mathfrak{s l}_{3}\right)$-Mod of finite-dimensional (left) $\mathcal{Q}_{\eta}$-modules (of type 1 ) is far from being semisimple. However, it has a semisimple quotient $\mathcal{Q}_{e}$, which is roughly obtained by killing the so-called tilting modules of quantum dimension zero. We refer to [AP95] for details, but all the reader needs to know for our purposes is that all $\mathrm{U}_{\mathrm{q}}\left(\mathfrak{s l}_{3}\right)$-modules $\mathrm{L}_{m, n}$ with $0 \leq m+n \leq e$ can also be regarded as irreducible $\mathrm{U}_{\eta}\left(\mathfrak{s l}_{3}\right)$-modules. Moreover,

$$
\left\{\left[\mathrm{L}_{m, n}\right] \mid 0 \leq m+n \leq e\right\}, \quad\left\{\left[\mathrm{X}^{k} \mathrm{Y}^{l}\right] \mid 0 \leq k+l \leq e\right\},
$$

are bases of $\left[\mathcal{Q}_{e}\right]_{\oplus}$ and $\left[\mathcal{Q}_{e}\right]_{\oplus}^{\mathbb{C}}$, and the quantum fusion product endows $\mathcal{Q}_{e}$ with the structure of a monoidal category, so $\left[\mathcal{Q}_{e}\right]_{\oplus}$ is a ring and $\left[\mathcal{Q}_{e}\right]_{\oplus}^{\mathbb{C}}$ is an algebra.

Note also that the rank of $\left[\mathcal{Q}_{e}\right]_{\oplus}$, respectively the dimension of $\left[\mathcal{Q}_{e}\right]_{\oplus}^{\mathbb{C}}$, is equal to the triangular number

$$
t_{e}=\frac{(e+1)(e+2)}{2}
$$

which follows from the fact that $\mathcal{Q}_{e}$ is only supported on a triangular cut-off of the positive Weyl chamber of $\mathfrak{s l}_{3}$, cf. (2-1). Said otherwise, since $\mathcal{Q}_{e}$ is semisimple, we have

$$
\left[\mathcal{Q}_{e}\right]_{\oplus}^{\mathbb{C}} \cong \mathbb{C}^{t_{e}}
$$

as vector spaces.

2B. Chebyshev-like polynomials for $\mathfrak{s l}_{3}$. We now recall certain polynomials introduced in the context of orthogonal polynomials by Koornwinder [Koo74], but phrased in a more convenient way for our purposes.

The $\mathfrak{s l}_{3}$-polynomials. Consider the polynomial ring $\mathbb{Z}[\mathrm{X}, \mathrm{Y}]$, in which $\mathrm{X}$ and $\mathrm{Y}$ from $(2-2)$ are treated as formal variables.

Definition 2.5. For each $m, n$ we define $\mathrm{U}_{m, n}(\mathrm{X}, \mathrm{Y}) \in \mathbb{Z}[\mathrm{X}, \mathrm{Y}]$ by

$$
\mathrm{U}_{m, n}(\mathrm{X}, \mathrm{Y})=\sum_{k, l} d_{m, n}^{k, l} \cdot \mathrm{X}^{k} \mathrm{Y}^{l}
$$

with $d_{m, n}^{k, l} \in \mathbb{Z}$ as in (2-3).

For fixed $e$, we often consider all the polynomials $\mathrm{U}_{m, n}(\mathrm{X}, \mathrm{Y})$ with $m+n=e+1$ together, cf. Example 2.6.

Example 2.6. The first few of these polynomials are $\mathrm{U}_{0,0}(\mathrm{X}, \mathrm{Y})=1$ and:

$$
\begin{aligned}
& \begin{array}{lll}
e=0 & \mathrm{U}_{1,0}(\mathrm{X}, \mathrm{Y})=\mathrm{X}, \mathrm{U}_{0,1}(\mathrm{X}, \mathrm{Y})=\mathrm{Y},
\end{array} \\
& e=1 \quad \quad \mathrm{U}_{2,0}(\mathrm{X}, \mathrm{Y})=\mathrm{X}^{2}-\mathrm{Y}, \mathrm{U}_{1,1}(\mathrm{X}, \mathrm{Y})=\mathrm{XY}-1, \mathrm{U}_{0,2}(\mathrm{X}, \mathrm{Y})=\mathrm{Y}^{2}-\mathrm{X}, \\
& e=2 \quad \mathrm{U}_{3,0}(\mathrm{X}, \mathrm{Y})=\mathrm{X}^{3}-2 \mathrm{XY}+1, \mathrm{U}_{2,1}(\mathrm{X}, \mathrm{Y})=\mathrm{X}^{2} \mathrm{Y}-\mathrm{Y}^{2}-\mathrm{X}, \\
& e=2 \quad \mathrm{U}_{1,2}(\mathrm{X}, \mathrm{Y})=\mathrm{XY}^{2}-\mathrm{X}^{2}-\mathrm{Y}, \mathrm{U}_{0,3}(\mathrm{X}, \mathrm{Y})=\mathrm{Y}^{3}-2 \mathrm{XY}+1, \\
& \mathrm{U}_{4,0}(\mathrm{X}, \mathrm{Y})=\mathrm{X}^{4}-3 \mathrm{X}^{2} \mathrm{Y}+\mathrm{Y}^{2}+2 \mathrm{X}, \quad \mathrm{U}_{3,1}(\mathrm{X}, \mathrm{Y})=\mathrm{X}^{3} \mathrm{Y}-2 \mathrm{XY}^{2}-\mathrm{X}^{2}+2 \mathrm{Y}, \\
& e=3 \quad \mathrm{U}_{2,2}(\mathrm{X}, \mathrm{Y})=\mathrm{x}^{2} \mathrm{Y}^{2}-\mathrm{x}^{3}-\mathrm{Y}^{3}, \\
& \mathrm{U}_{1,3}(\mathrm{X}, \mathrm{Y})=\mathrm{XY}^{3}-2 \mathrm{X}^{2} \mathrm{Y}-\mathrm{Y}^{2}+2 \mathrm{X}, \quad \mathrm{U}_{0,4}(\mathrm{X}, \mathrm{Y})=\mathrm{Y}^{4}-3 \mathrm{XY}^{2}+\mathrm{X}^{2}+2 \mathrm{Y}, \\
& \mathrm{U}_{5,0}(\mathrm{X}, \mathrm{Y})=\mathrm{X}^{5}-4 \mathrm{X}^{3} \mathrm{Y}+3 \mathrm{XY}^{2}+3 \mathrm{X}^{2}-2 \mathrm{Y}, \quad \mathrm{U}_{4,1}(\mathrm{X}, \mathrm{Y})=\mathrm{X}^{4} \mathrm{Y}-3 \mathrm{X}^{2} \mathrm{Y}^{2}-\mathrm{X}^{3}+\mathrm{Y}^{3}+4 \mathrm{XY}-1, \\
& e=4 \quad \mathrm{U}_{3,2}(\mathrm{X}, \mathrm{Y})=\mathrm{X}^{3} \mathrm{Y}^{2}-\mathrm{X}^{4}-2 \mathrm{XY}^{3}+\mathrm{X}^{2} \mathrm{Y}+2 \mathrm{Y}^{2}-\mathrm{X}, \quad \mathrm{U}_{2,3}(\mathrm{X}, \mathrm{Y})=\mathrm{X}^{2} \mathrm{Y}^{3}-\mathrm{Y}^{4}-2 \mathrm{X}^{3} \mathrm{Y}+\mathrm{XY}^{2}+2 \mathrm{X}^{2}-\mathrm{Y}, \\
& \mathrm{U}_{1,4}(\mathrm{X}, \mathrm{Y})=\mathrm{XY}^{4}-3 \mathrm{X}^{2} \mathrm{Y}^{2}-\mathrm{Y}^{3}+\mathrm{X}^{3}+4 \mathrm{XY}-1, \mathrm{U}_{0,5}(\mathrm{X}, \mathrm{Y})=\mathrm{Y}^{5}-4 \mathrm{XY}^{3}+3 \mathrm{X}^{2} \mathrm{Y}+3 \mathrm{Y}^{2}-2 \mathrm{X} .
\end{aligned}
$$

Note that the ones with $m+n=e+1$ correspond to the $e+1$-line in (2-1).

By convention, $\mathrm{U}_{m, n}(\mathrm{X}, \mathrm{Y})$ and $\mathrm{L}_{m, n}$ with negative subscripts $m$ or $n$ are zero. 
Lemma 2.7. We have the following Chebyshev-like recursion relations

$$
\begin{gathered}
\mathrm{U}_{m, n}(\mathrm{X}, \mathrm{Y})=\mathrm{U}_{n, m}(\mathrm{Y}, \mathrm{X}), \\
\mathrm{XU}_{m, n}(\mathrm{X}, \mathrm{Y})=\mathrm{U}_{m+1, n}(\mathrm{X}, \mathrm{Y})+\mathrm{U}_{m-1, n+1}(\mathrm{X}, \mathrm{Y})+\mathrm{U}_{m, n-1}(\mathrm{X}, \mathrm{Y}), \\
\mathrm{YU}_{m, n}(\mathrm{X}, \mathrm{Y})=\mathrm{U}_{m, n+1}(\mathrm{X}, \mathrm{Y})+\mathrm{U}_{m+1, n-1}(\mathrm{X}, \mathrm{Y})+\mathrm{U}_{m-1, n}(\mathrm{X}, \mathrm{Y}) .
\end{gathered}
$$

Together with the starting conditions for $e=0,1$ as in Example 2.6, these recursion relations determine the polynomials $\mathrm{U}_{m, n}(\mathrm{X}, \mathrm{Y})$ for all $m, n$.

Proof. The relation $\mathrm{U}_{m, n}(\mathrm{X}, \mathrm{Y})=\mathrm{U}_{n, m}(\mathrm{Y}, \mathrm{X})$ boils down to $\mathrm{X} \cong \mathrm{Y}^{*}$. Moreover, by standard results in the representation theory of $\mathfrak{s l}_{3}$, we obtain

$$
\begin{aligned}
& \mathrm{X} \otimes \mathrm{L}_{m, n} \cong \mathrm{L}_{m+1, n} \oplus \mathrm{L}_{m-1, n+1} \oplus \mathrm{L}_{m, n-1}, \\
& \mathrm{Y} \otimes \mathrm{L}_{m, n} \cong \mathrm{L}_{m, n+1} \oplus \mathrm{L}_{m+1, n-1} \oplus \mathrm{L}_{m-1, n},
\end{aligned}
$$

which proves the two recursions.

Lemma 2.8. The polynomial $\mathrm{U}_{m, n}(\mathrm{X}, \mathrm{Y})$ has a constant term if and only if $(m \equiv n \not \equiv 2) \bmod 3$. This constant term is equal to 1 if $m \equiv n \equiv 0 \bmod 3$, and equal to -1 if $m \equiv n \equiv 1 \bmod 3$.

Proof. The claim follows inductively from Example 2.6 and Lemma 2.7.

Their complex roots. The following definition will be crucial for us.

Definition 2.9. For fixed level $e$, let $\mathrm{J}_{e}$ be the ideal generated by

$$
\left\{\mathrm{U}_{m, n}(\mathrm{X}, \mathrm{Y}) \mid m+n=e+1\right\} \subset \mathbb{Z}[\mathrm{X}, \mathrm{Y}] .
$$

We call $\mathrm{J}_{e}$ the vanishing ideal of level $e$. Associated to it is the vanishing set of level $e$

$$
\mathrm{V}_{e}=\left\{(\alpha, \beta) \in \mathbb{C}^{2} \mid p(\alpha, \beta)=0 \text { for all } p \in \mathrm{J}_{e}\right\} \subset \mathbb{C}^{2}
$$

which we consider as a complex variety.

Since $\mathrm{X}$ and $\mathrm{Y}$ generate $\mathcal{Q}_{e}$, we have

$$
\left[\mathcal{Q}_{e}\right]_{\oplus}^{\mathbb{C}} \cong \mathbb{C}[\mathrm{X}, \mathrm{Y}] / \mathrm{J}_{e} \cong \mathbb{C}^{t_{e}},
$$

as vector spaces, where $t_{e}=\frac{(e+1)(e+2)}{2}$ denotes the triangular number. Note that the left isomorphism in (2-8) is actually an isomorphism of algebras, which follows from the explicit form of the fusion rules for $\mathcal{Q}_{e}$ (which can be deduced from e.g. [Saw06, Corollary 8] or [Sch17, Proposition 3.2.2]).

Using this, we can compute $\# \mathrm{~V}_{e}$, the number of points in $\mathrm{V}_{e}$, i.e. the number of common roots of the polynomials in $\mathrm{J}_{e}$.

Lemma 2.10. We have $\# \mathrm{~V}_{e}=t_{e}$.

Before we prove Lemma 2.10, let us fix some notation for complex numbers: i denotes $\sqrt{-1}$ (in the positive upper half-plane), $\zeta=\exp \left(2 \pi \mathrm{i} \frac{1}{3}\right)$ and $\bar{z}$ will denote the complex conjugate of a complex number $z \in \mathbb{C}$.

Proof. By (2-8) and a corollary of Hilbert's Nullstellensatz [Ful89, Corollary I.7.4], we immediately see that $\# \mathrm{~V}_{e} \leq t_{e}$,

To see the equality, consider the following functions, due to [Koo74]:

$$
\begin{aligned}
Z: \mathbb{C}^{2} \rightarrow \mathbb{C}, \quad Z(\sigma, \tau)= & \exp (\mathrm{i} \sigma)+\exp (-\mathrm{i} \tau)+\exp (\mathrm{i}(-\sigma+\tau)), \\
E_{a, b}^{-}: \mathbb{C}^{2} \rightarrow \mathbb{C}, \quad E_{a, b}^{-}(\sigma, \tau)= & \exp (\mathrm{i}(a \sigma+b \tau))-\exp (\mathrm{i}((a+b) \sigma-b \tau)) \\
& +\exp (\mathrm{i}(-(a+b) \sigma+a \tau))-\exp (\mathrm{i}(-b \sigma-a \tau)) \\
& +\exp (\mathrm{i}(b \sigma-(a+b) \tau))-\exp (\mathrm{i}(-a \sigma+(a+b) \tau)),
\end{aligned}
$$

where $a, b \in \mathbb{N}^{0}$. 
The functions $Z$ and $E_{a, b}^{-}$are clearly $2 \pi$-periodic in both variables, i.e. they define functions on a 2-torus $\mathrm{T}^{2}$. As one easily checks, they are invariant under the reflection $(\sigma, \tau) \mapsto(-\sigma+\tau, \tau)$ and antiinvariant under the reflection $(\sigma, \tau) \mapsto(\sigma, \sigma-\tau)$, which generate the symmetric group $S_{3}$. The fundamental domain of the quotient $\mathrm{T}^{2} / S_{3}$ is equal to

$$
D=\left\{(\sigma, \tau) \mid 0 \leq \sigma+\tau \leq 2 \pi, \frac{\sigma}{2} \leq \tau \leq 2 \sigma\right\} .
$$

Note that all zeros of $E_{1,1}^{-}$lie on the boundary of $D$. Therefore $Z$ and $E_{m+1, n+1}^{-} / E_{1,1}^{-}$define functions on the interior of $D$.

As explained in [Koo74], $Z$ and its complex conjugate $\bar{Z}$ map $D$ bijectively onto the (3-cusps) discoid $\mathrm{d}_{3}=\left\{z=(x, y) \in \mathbb{C} \mid-z^{2} \bar{z}^{2}+4 z^{3}+\bar{z}^{3}-18 z \bar{z}+27 \geq 0\right\}$ bounded by the deltoid curve $\mathrm{d}=\{z=2 \exp (\mathrm{it})+\exp (-2 \mathrm{i} t) \mid t \in[0,2 \pi]\}$ (also called Steiner's hypocycloid):

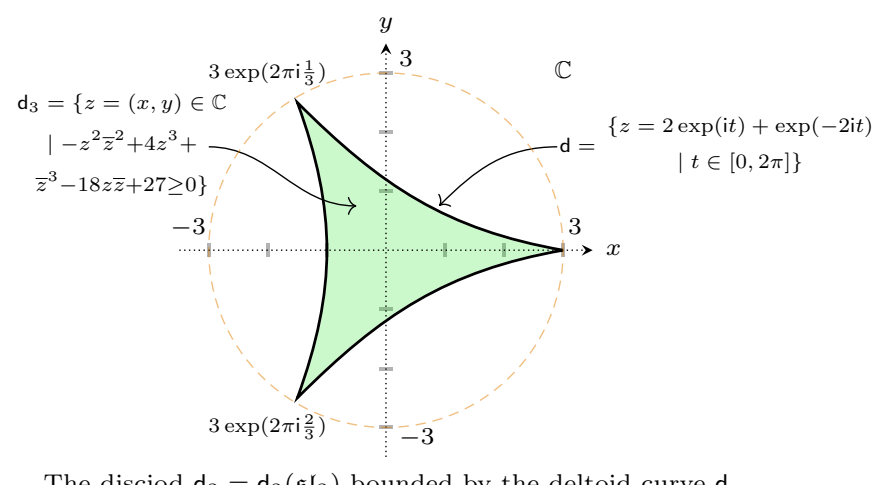

The disciod $d_{3}=d_{3}\left(\mathfrak{s l}_{3}\right)$ bounded by the deltoid curve $d$

The discoid $\mathrm{d}_{3}$ has a $\mathbb{Z} / 3 \mathbb{Z}$-symmetry, given by $(z, \bar{z}) \mapsto\left(\zeta^{ \pm 1} z, \zeta^{\mp 1} \bar{z}\right)$, and its singularities are the primitive, complex third roots of unity multiplied by 3 .

For any $a, b \in \mathbb{N}^{0}$, the zeros of $E_{a, b}^{-}$are known, cf. [EP10, Section 7.1]. However, let us give an independent proof.

'Lemma 2.10.Claim'. Let $a, b \in \mathbb{N}^{0}, a+b=s \geq 2$. Then $E_{a, b}^{-}(\sigma, \tau)=0$ if

$$
(\sigma, \tau)=\left(\frac{2 \pi(2 c+d+3)}{3 s}, \frac{2 \pi(c+2 d+3)}{3 s}\right), \quad \text { with } c, d \in \mathbb{N}^{0} .
$$

Proof of 'Lemma 2.10.Claim'. We have

$$
\zeta^{\frac{a(2 c+d)+b(c+2 d)}{s}}=\zeta^{\frac{a(2 c+d)+b(c+2 d)-3(a+b)(c+d)}{s}}=\zeta^{\frac{-b(2 c+d)-a(c+2 d)}{s}},
$$

using that $a+b=s,(2 c+d)+(c+2 d)=3(c+d)$ and $\zeta^{3}=1$. Similarly, we obtain

$$
\begin{gathered}
\zeta^{\frac{(a+b)(2 c+d+3)-b(c+2 d+3)}{s}}=\zeta^{\frac{(a+b)(2 c+d+3)-b(c+2 d+3)+3(a+b)(c+d+2)}{s}} \\
=\zeta^{\frac{2(a+b)(2 c+d+3)+a(c+2 d+3)}{s}}=\zeta^{\frac{-(a+b)(2 c+d+3)+a(c+2 d+3)}{s}} . \\
\zeta^{\frac{b(2 c+d+3)-(a+b)(c+2 d+3)}{s}}=\zeta^{\frac{b(2 c+d+3)-(a+b)(c+2 d+3)-3(a+b)(c+d+2)}{s}} \\
=\zeta^{\frac{-a(2 c+d+3)-2(a+b)(c+2 d+3)}{s}}=\zeta^{\frac{-a(2 c+d+3)+(a+b)(c+2 d+3)}{s}} .
\end{gathered}
$$

This gives $E_{a, b}^{-}(\sigma, \tau)=0$ for $(\sigma, \tau)$ as in (2-10), and completes the proof of 'Lemma 2.10.Claim'.

Next, for any $m, n$, we have

$$
\mathrm{U}_{m, n}(Z(\sigma, \tau), \overline{Z(\sigma, \tau)})=E_{m+1, n+1}^{-}(\sigma, \tau) / E_{1,1}^{-}(\sigma, \tau) .
$$

Let $(\sigma, \tau)$ be as in (2-10) with $a=m+1$ and $b=n+1$, and assume $(\sigma, \tau)$ is in the interior of $D$. Then we have $\mathrm{U}_{Z(\sigma, \tau), \overline{Z(\sigma, \tau)}}(\mathrm{X}, \mathrm{Y}) m, n=0$ by 'Lemma 2.10.Claim'. 
To make the connection with our notation from before, let $m+n=e+1$ and $k=c, l=d$. By the above, for all

$$
(\sigma, \tau)=\left(\frac{2 \pi(2 k+l+3)}{3(e+3)}, \frac{2 \pi(k+2 l+3)}{3(e+3)}\right) \quad \text { with } 0 \leq k+l \leq e,
$$

we have $\mathrm{U}_{m, n}(Z(\sigma, \tau), \overline{Z(\sigma, \tau)})=0$.

Thus, $\# \mathrm{~V}_{e} \geq \#\left\{(k, l) \in X^{+} \mid 0 \leq k+l \leq e\right\}=t_{e}$. Since we already know that $\# \mathrm{~V}_{e} \leq t_{e}$, equality must hold.

Remark 2.11. Applying $Z$ to (2-11) gives the precise form of the elements of $\mathrm{V}_{e}$ :

$$
\mathrm{V}_{e}=\left\{(\alpha, \beta) \in \mathbb{C}^{2} \mid \alpha=Z(\sigma, \tau), \beta=\overline{Z(\sigma, \tau)}\right\}
$$

for $(\sigma, \tau)$ as in (2-11). For a fixed level, the common roots of the polynomials $\mathrm{U}_{m, n}(\mathrm{X}, \mathrm{Y})$ all lie in the interior of the discoid from (2-9).

Example 2.12. The polynomials for $e=1,2,3$ are given in Example 2.6. The first (or $\mathrm{X}$ ) entries of their common zeros are

$$
\begin{gathered}
e=1:\left\{\text { roots of }(X-1)\left(X^{2}+X+1\right)\right\}, \\
e=2:\left\{\text { roots of }\left(X^{2}-X-1\right)\left(X^{4}+X^{3}+2 X^{2}-X+1\right)\right\}, \\
e=3:\left\{\text { roots of } X(X-2)\left(X^{2}+2 X+4\right)\left(X^{6}-X^{3}+1\right)\right\} .
\end{gathered}
$$

The second (or $\mathrm{Y}$ ) entries are the complex conjugates. Plotted into $\mathbb{C}$ one gets

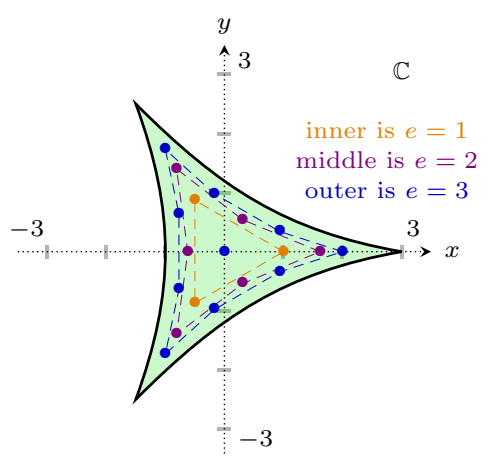

Letting $e \gg 0$, these approximate the deltoid curve $\mathrm{d}$ (layer-wise).

\section{Trihedral Hecke algebras}

As before, $k, l, m, n$ etc. will be non-negative integers, and $e$ will denote the level. We are now going to introduce the trihedral Hecke algebras. The reader possibly spots the analogies with the dihedral Hecke algebra right away, but, for completeness, we have also listed some of them in Section 3D.

3A. Some color conventions. Throughout we will use the set of primary colors $B R Y=$ $\{b, r, y\}$, the elements of which are blue $b$, red $r$ and yellow $y$, the set of secondary colors $G O P=\{g, o, p\}$, the elements of which are green $g=\{b, y\}$, orange $o=\{y, r\}$ and purple $p=\{b, r\}$, and the color white $\emptyset$. We also use dummy colors $\mathbf{u}, \mathbf{v} \in G O P$, and from now on $\mathbf{u}, \mathbf{v}$, etc. will always denote arbitrary elements in $G O P$.

Moreover, we fix a cyclic ordering, and its inverse, of the secondary colors:

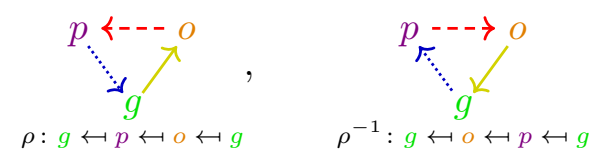

Note that we usually read from right to left, i.e. we use the operator notation. 
The action of $\rho$ on GOP can be read off from (3-1): $\rho(g)=o, \rho(o)=p$ and $\rho(p)=g$, and $\rho^{k-l}$ only depends on $(k-l) \bmod 3$, for any $k, l$.

3B. The trihedral Hecke algebra of level $\infty$. In this section and in Section 3C, we work over $\mathbb{C}_{\mathrm{v}}=\mathbb{C}(\mathrm{v})$, with $\mathrm{v}$ being a generic parameter.

The underlying Coxeter group. Let $\mathrm{W}$ be the Coxeter group of affine type $\mathrm{A}_{2}$, generated by three reflections that we denote by $b, r$, y, i.e.

$$
\sum_{b}^{\tilde{A}_{2}}{ }_{3}^{r} \rightsquigarrow \mathrm{W}=\left\langle b, r, y \mid b^{2}=r^{2}=y^{2}=1, b r b=r b r, b y b=y b y, r y r=y r y\right\rangle .
$$

In order to simplify the notation, we identify the vertices in the Coxeter diagram of $\mathrm{W}$ with the corresponding reflections.

Moreover, let $g$, o and $p$ be the maximal proper parabolic subsets, and let $\mathbf{W}_{g}, \mathbf{W}_{o}$ and $\mathrm{W}_{p}$ be the corresponding standard parabolic subgroups of $\mathrm{W}$, which are all isomorphic to the (finite) type $\mathrm{A}_{2}$ Weyl group. Furthermore, let

$$
w_{g}=b y b=y b y \in \mathrm{W}_{g}, \quad w_{o}=r y r=y r y \in \mathrm{W}_{o}, \quad w_{p}=b r b=r b r \in \mathrm{W}_{p}
$$

denote the longest elements in these parabolic subgroups.

The trihedral Hecke algebra. We define now the trihedral Hecke algebra of level $\infty$.

Definition 3.1. Let $\mathrm{T}_{\infty}$ be the associative, unital $\left(\mathbb{C}_{\mathrm{v}^{-}}\right)$algebra generated by three elements $\theta_{g}, \theta_{o}, \theta_{p}$ subject to the following relations.

$$
\begin{gathered}
\theta_{g}^{2}=[3]_{\mathrm{v}} ! \theta_{g}, \quad \theta_{o}^{2}=[3]_{\mathrm{v}} ! \theta_{o}, \quad \theta_{p}^{2}=[3]_{\mathrm{v}} ! \theta_{p}, \\
\theta_{g} \theta_{o} \theta_{g}=\theta_{g} \theta_{p} \theta_{g}, \quad \theta_{o} \theta_{g} \theta_{o}=\theta_{o} \theta_{p} \theta_{o}, \quad \theta_{p} \theta_{g} \theta_{p}=\theta_{p} \theta_{o} \theta_{p} .
\end{gathered}
$$

Here, $[3]_{\mathrm{v}}$ ! is the $\mathrm{v}$-factorial from (1-1).

Let $H_{v}=H_{v}\left(\tilde{A}_{2}\right)$ denote the Hecke algebra of affine type $A_{2}$, with the same conventions as in [Soe97, Section 2]. Recall that $\mathrm{H}_{\mathrm{v}}$ can be defined as the associative, unital $\left(\mathbb{C}_{\mathrm{v}}\right)$-algebra generated by $\theta_{b}, \theta_{r}$ and $\theta_{y}$ subject to

$$
\begin{array}{rrr}
\theta_{b}^{2}=[2]_{\mathrm{v}} \theta_{b}, & \theta_{y}^{2}=[2]_{\mathrm{v}} \theta_{y}, & \theta_{r}^{2}=[2]_{\mathrm{v}} \theta_{r}, \\
\left(\theta_{w_{g}}=\right) \theta_{b} \theta_{y} \theta_{b}-\theta_{b} & \left(\theta_{w_{o}}=\right) \theta_{r} \theta_{y} \theta_{r}-\theta_{r} & \left(\theta_{w_{p}}=\right) \theta_{b} \theta_{r} \theta_{b}-\theta_{b} \\
=\theta_{u} \theta_{b} \theta_{l}-\theta_{u}, & =\theta_{r} \theta_{r} \theta_{-}-\theta_{\vartheta}, & =\theta_{r} \theta_{b} \theta_{r}-\theta_{r} .
\end{array}
$$

For any $w \in \mathrm{W}$, let $\theta_{w}$ be the corresponding Kazhdan-Lusztig (KL for short) basis element of $\mathrm{H}_{\mathrm{v}}$, e.g. the expression $\theta_{w_{\mathbf{u}}}$ in (3-6). (Note that $\theta_{w}$ is denoted $\underline{H}_{w}$ in [Soe97, Section 2], while the standard basis is denoted $H_{w}$ therein.)

Lemma 3.2. The algebra homomorphism given by

$$
\theta_{g} \mapsto \theta_{w_{g}}, \quad \theta_{o} \mapsto \theta_{w_{o}}, \quad \theta_{p} \mapsto \theta_{w_{p}},
$$

defines an embedding $\mathrm{T}_{\infty} \hookrightarrow \mathrm{H}_{\mathrm{v}}$ of algebras.

Proof. By $(3-5),(3-6)$ and the identity $[2]_{\mathrm{v}}^{3}-[2]_{\mathrm{v}}=[2]_{\mathrm{v}}[3]_{\mathrm{v}}$, we obtain

$$
\theta_{w_{g}}^{2}=[3]_{\mathrm{v}} ! \theta_{w_{g}}, \quad \theta_{w_{o}}^{2}=[3]_{\mathrm{v}} ! \theta_{w_{o}}, \quad \theta_{w_{p}}^{2}=[3]_{\mathrm{v}} ! \theta_{w_{p}} .
$$

This shows that (3-3) holds in $\mathrm{H}_{\mathrm{v}}$.

Proving (3-4) is harder. Let us indicate how to prove $\theta_{w_{g}} \theta_{w_{o}} \theta_{w_{g}}=\theta_{w_{g}} \theta_{w_{p}} \theta_{w_{g}}$. (The other two follow by exchanging colors.) By (3-6), this is equivalent to proving

$$
\left(\theta_{b} \theta_{y} \theta_{b}-\theta_{b}\right)\left(\theta_{b} \theta_{r} \theta_{b}-\theta_{b}\right)\left(\theta_{b} \theta_{y} \theta_{b}-\theta_{b}\right)=\left(\theta_{y} \theta_{b} \theta_{y}-\theta_{y}\right)\left(\theta_{y} \theta_{r} \theta_{y}-\theta_{y}\right)\left(\theta_{y} \theta_{b} \theta_{y}-\theta_{y}\right) \text {. }
$$


By (3-5), the right-hand side in (3-7) is equal to

$$
\begin{gathered}
{[2]_{\mathbf{v}}^{2}\left(\theta_{y} \theta_{b} \theta_{y} \theta_{r} \theta_{y} \theta_{b} \theta_{y}-\theta_{y} \theta_{b} \theta_{y} \theta_{r} \theta_{y}-\theta_{y} \theta_{r} \theta_{y} \theta_{b} \theta_{y}-\theta_{y} \theta_{b} \theta_{y} \theta_{b} \theta_{y}+2 \theta_{y} \theta_{b} \theta_{y}+\theta_{y} \theta_{r} \theta_{y}-\theta_{y}\right)} \\
\stackrel{(3-6)}{=}[2]_{\mathbf{v}}^{2}\left(\theta_{y} \theta_{b} \theta_{y} \theta_{r} \theta_{y} \theta_{b} \theta_{y}-\theta_{y} \theta_{b} \theta_{y} \theta_{r} \theta_{y}-\theta_{y} \theta_{r} \theta_{y} \theta_{b} \theta_{y}-[3]_{\mathbf{v}} \theta_{w_{g}}+\theta_{y} \theta_{r} \theta_{y}\right)
\end{gathered}
$$

Similarly, the left-hand side in (3-7) is equal to

$$
[2]_{\mathrm{v}}^{2}\left(\theta_{b} \theta_{y} \theta_{b} \theta_{r} \theta_{b} \theta_{y} \theta_{b}-\theta_{b} \theta_{y} \theta_{b} \theta_{r} \theta_{b}-\theta_{b} \theta_{r} \theta_{b} \theta_{y} \theta_{b}-[3]_{\mathrm{v}} \theta_{w_{g}}+\theta_{b} \theta_{r} \theta_{b}\right) .
$$

One can obtain (3-9) from (3-8) by systematically using (3-6) and the fact that $w_{g}, w_{o}, w_{p}$ have two equivalent expressions each. For example, by (3-6), we have $\theta_{y} \theta_{b} \theta_{y}=\theta_{b} \theta_{y} \theta_{b}+\theta_{b}-\theta_{y}$. Using this to rewrite the first term in (3-8) and carefully continuing in this way yields the claimed equality.

Finally, using an appropriate integral form, $\mathrm{H}_{\mathrm{v}}$ specializes to $\mathbb{C}[\mathrm{W}]$ for $\mathrm{v}=1$. Moreover, recall that $\mathbb{C}[\mathrm{W}]$ has a faithful representation $\mathrm{P}_{1}$, which is induced by the regular $\mathrm{W}$-action on the set of alcoves obtained from the hyperplane arrangement associated to $\mathrm{W}$, and that $\mathrm{P}_{1}$ can be v-deformed to $\mathrm{P}_{\mathrm{v}}$, cf. [Soe97, Section 4 and Lemma 4.1]. The v-deformation $\mathrm{P}_{\mathrm{v}}$ stays faithful: Each standard basis element $H_{w} \in \mathbb{C}[\mathrm{W}]$ is mapped to a different $\mathbb{C}$-linear operator by $\mathrm{P}_{1}$, so each $\mathrm{KL}$ basis element $\theta_{w} \in \mathrm{H}_{\mathrm{v}}$ is mapped to a different $\mathbb{C}_{\mathrm{v}}$-linear operator by $\mathrm{P}_{\mathrm{v}}$, due to the particular form of the change of basis

$$
\theta_{w} \in H_{w}+\sum_{w^{\prime} \leq_{\mathrm{B}} w} \mathrm{v} \mathbb{Z}[\mathrm{v}] H_{w^{\prime}} .
$$

Here $\leq_{\mathrm{B}}$ is the Bruhat order, see e.g. [Soe97, Claim 2.3]. By pulling back $\mathrm{P}_{\mathrm{v}}$ to $\mathrm{T}_{\infty}$ along the algebra homomorphism in this lemma, injectivity of the latter follows from the faithfulness of the representation.

The trihedral Kazhdan-Lusztig combinatorics. We are going to define a quotient of $\mathrm{T}_{\infty}$. In order to do that, we first have to introduce certain elements. For any $k, l, \mathbf{u}$, let

$$
\mathrm{h}_{\mathbf{u}}^{k, l}=\mathrm{h}_{\mathbf{u}}^{k, l}(\theta)=\theta_{\mathbf{u}_{k+l}} \cdots \theta_{\mathbf{u}_{1}} \theta_{\mathbf{u}_{0}}
$$

where $\mathbf{u}_{i}$ for all $0 \leq i \leq k+l$ is given by $\mathbf{u}_{0}=\mathbf{u}, \mathbf{u}_{i+1}=\rho\left(\mathbf{u}_{i}\right)$ for (any) $k$ values of $i$, and $\mathbf{u}_{i+1}=\rho^{-1}\left(\mathbf{u}_{i}\right)$ for the remaining values of $i$. Note that

$$
\mathrm{h}_{\mathbf{u}}^{0,0}=\theta_{\mathbf{u}} \quad \text { for any } \mathbf{u} .
$$

Moreover, by convention, $\mathrm{h}_{\mathbf{u}}^{k, l}=0$ in case $k$ or $l$ are negative. We call $\mathbf{u}$ the (right) starting color of $\mathrm{h}_{\mathbf{u}}^{k, l}$. The fact that $\mathrm{h}_{\mathbf{u}}^{k, l}$ is well-defined is established by the following lemma.

Lemma 3.3. For any $k, l, \mathbf{u}$, the element $\mathrm{h}_{\mathbf{u}}^{k, l}$ only depends on $k$ and $l$, not on the chosen sequence $\mathbf{u}_{k+l}, \cdots, \mathbf{u}_{1}, \mathbf{u}_{0}=\mathbf{u}$.

Proof. Any word representing $\mathrm{h}_{\mathbf{u}}^{k, l}$ involves $k$ counterclockwise rotations and $l$ clockwise rotations of $G O P$. The relations (3-4) translate into $\rho \rho^{-1}=\rho^{-1} \rho=$ id. Therefore, any word representing $\mathrm{h}_{\mathbf{u}}^{k, l}$ is equivalent to the word $\theta_{\mathbf{u}_{k+l}} \cdots \theta_{\mathbf{u}_{0}}$ such that $\mathbf{u}_{0}=\mathbf{u}$ and

$$
\mathbf{u}_{r}=\rho\left(\mathbf{u}_{r-1}\right), \text { for all } 1 \leq r \leq k, \quad \mathbf{u}_{r}=\rho^{-1}\left(\mathbf{u}_{r-1}\right), \text { for all } k+1 \leq r \leq k+l,
$$

which concludes the proof.

Similarly, we can define

$$
{ }_{\mathbf{v}}^{k, l} \mathrm{~h}=\mathrm{h}_{\mathbf{u}}^{k, l} \quad \text { such that } \mathbf{v}=\rho^{k-l}(\mathbf{u}),
$$

for $k, l, \mathbf{v}$. Lemma 3.3 implies, mutatis mutandis, that $k_{\mathbf{v}}, \mathrm{h}$ is also independent of the chosen sequence $\mathbf{v}=\mathbf{u}_{k+l}, \ldots, \mathbf{u}_{0}$. 
Remark 3.4. We can view $\mathrm{X}$ and $\mathrm{Y}$ as acting via counterclockwise respectively clockwise rotation of (3-1). By Lemma 3.3, we can view the elements $\mathrm{h}_{\mathbf{u}}^{k, l}$ as being associated to $\mathrm{X}^{k} \mathrm{Y}^{l}$ (after fixing a starting color $\mathbf{u}$ ), because its definition involves $k$ times the application of $\rho$ and $l$ times that of $\rho^{-1}$. Lemma 3.3 then translates into the equality $\mathrm{XY}=\mathrm{YX}$.

Example 3.5. Let us fix $g$ as a starting color. Then

$$
\begin{array}{ccc}
\mathrm{h}_{g}^{2,0}=\theta_{p} \theta_{o} \theta_{g}, & \mathrm{~h}_{g}^{1,1}=\theta_{g} \theta_{p} \theta_{g}=\theta_{g} \theta_{o} \theta_{g}, & \mathrm{~h}_{g}^{0,2}=\theta_{o} \theta_{p} \theta_{g}, \\
\longleftrightarrow \mathrm{X}^{2} \mathrm{Y}^{0}, & \leadsto \mathrm{X}^{1} \mathrm{Y}^{1}=\mathrm{Y}^{1} \mathrm{X}^{1}, & \mathrm{X}^{0} \mathrm{Y}^{2}
\end{array}
$$

where we think of the color changes $g \leftrightarrow p \leftrightarrow o \hookleftarrow g$ as corresponding to multiplication by $\mathrm{X}$, and the color changes $g \hookleftarrow o \hookleftarrow p \leftrightarrow g$ as corresponding to multiplication by Y.

Recall that $d_{m, n}^{k, l}$ denote the numbers from Section 2, coming from the representation theory of $\mathfrak{s l}_{3}$. For each pair $m, n$, we define three colored KL basis elements:

$$
\begin{gathered}
\mathrm{c}_{g}^{m, n}=\sum_{k, l}[2]_{\mathrm{v}}^{-k-l} d_{m, n}^{k, l} \mathrm{~h}_{g}^{k, l}, \quad \mathrm{c}_{o}^{m, n}=\sum_{k, l}[2]_{\mathrm{v}}^{-k-l} d_{m, n}^{k, l} \mathrm{~h}_{o}^{k, l}, \\
\mathrm{c}_{p}^{m, n}=\sum_{k, l}[2]_{\mathrm{v}}^{-k-l} d_{m, n}^{k, l} \mathrm{~h}_{p}^{k, l} .
\end{gathered}
$$

Note that the three sums are finite, because $d_{m, n}^{k, l}=0$ unless $k+l \leq m+n$, as mentioned after (2-3). Moreover, by convention, $\mathrm{c}_{\mathbf{u}}^{k, l}=0$ in case $k$ or $l$ are negative.

Furthermore, by (2-3) and Lemma $2.4, d_{m, n}^{k, l}=0$ if $k-l \not \equiv m-n \bmod 3$. This implies that, for any $m, n, \mathbf{u}$, all terms $\mathrm{h}_{\mathbf{u}}^{k, l}$ of $\mathrm{c}_{\mathbf{u}}^{m, n}$ in (3-12) have the same left-most factor $\theta_{\mathbf{v}}$, where $\mathbf{v}=\rho^{m-n}(\mathbf{u})$, by $(3-11)$. Therefore, we can also define

$$
{ }_{\mathbf{v}}^{m, n} \mathrm{c}=\mathrm{c}_{\mathbf{u}}^{m, n} \quad \text { such that } \mathbf{v}=\rho^{m-n}(\mathbf{u}) .
$$

We call $\mathrm{c}_{g}^{m, n}, \mathrm{c}_{o}^{m, n}$ and $\mathrm{c}_{p}^{m, n}$ the (right) colored KL elements. As before,

$$
c_{\mathbf{u}}^{0,0}=\theta_{\mathbf{u}} \quad \text { for any } \mathbf{u} .
$$

Example 3.6. For a fixed $\mathbf{u}$, the element $c_{\mathbf{u}}^{m, n}$ (or alternatively ${ }_{\mathbf{u}}^{m, n} \mathrm{c}$ ) is associated to the orthogonal polynomial $\mathrm{U}_{m, n}(\mathrm{X}, \mathrm{Y})$ from Section $2 \mathrm{~B}$, cf. Example 3.6. For example, fix $g$ as a starting color. Then

$$
\begin{aligned}
& \mathrm{c}_{g}^{2,0}=[2]_{\mathrm{v}}^{-2} \theta_{p} \theta_{o} \theta_{g}-[2]_{\mathrm{v}}^{-1} \theta_{p} \theta_{g} \quad \mathbf{c}_{g}^{1,1}=[2]_{\mathrm{v}}^{-2} \theta_{g} \theta_{p} \theta_{g}-\theta_{g} \quad \mathbf{c}_{g}^{0,2}=[2]_{\mathrm{v}}^{-2} \theta_{o} \theta_{p} \theta_{g}-[2]_{\mathrm{v}}^{-1} \theta_{o} \theta_{g} \\
& \leadsto \mathrm{U}_{2,0}(\mathrm{X}, \mathrm{Y})=\mathrm{X}^{2}-\mathrm{Y}, \leadsto \mathrm{U}_{1,1}(\mathrm{X}, \mathrm{Y})=\mathrm{XY}-1, \quad \leadsto \mathrm{U}_{0,2}(\mathrm{X}, \mathrm{Y})=\mathrm{Y}^{2}-\mathrm{X}
\end{aligned}
$$

Similarly for the other colors.

As we will see, Proposition 4.31 identifies the colored KL elements with the Grothendieck classes of the indecomposables in a certain 2-full 2-subcategory of singular Soergel bimodules. In particular, the next lemma and proposition need some notions from categorification which we only recall in Section 4. Consequently, we postpone their proofs until the end of Section 4.

Lemma 3.7. For all $m, n, \mathbf{u}, \mathbf{v}$, we have

$$
\theta_{\mathbf{u}} \mathbf{c}_{\mathbf{v}}^{m, n}= \begin{cases}{[3]_{\mathbf{v}} ! c_{\mathbf{v}}^{m, n},} & \text { if } \rho^{m-n}(\mathbf{u})=\mathbf{v}, \\ {[2]_{\mathbf{v}}\left(c_{\mathbf{v}}^{m+1, n}+c_{\mathbf{v}}^{m-1, n+1}+c_{\mathbf{v}}^{m, n-1}\right),} & \text { if } \rho^{m+1-n}(\mathbf{u})=\mathbf{v}, \\ {[2]_{\mathbf{v}}\left(c_{\mathbf{v}}^{m, n+1}+c_{\mathbf{v}}^{m+1, n-1}+c_{\mathbf{v}}^{m-1, n}\right),} & \text { if } \rho^{m-(n+1)}(\mathbf{u})=\mathbf{v},\end{cases}
$$

where terms with negative indices are zero.

By (3-13) and Lemma 3.7, we also have

$$
c_{\mathbf{v}}^{m, n} \theta_{\mathbf{u}}= \begin{cases}{[3]_{\mathbf{v}} ! c_{\mathbf{u}}^{m, n},} & \text { if } \mathbf{u}=\mathbf{v}, \\ {[2]_{\mathbf{v}}\left(\mathrm{c}_{\mathbf{u}}^{m+1, n}+\mathrm{c}_{\mathbf{u}}^{m-1, n+1}+\mathrm{c}_{\mathbf{u}}^{m, n-1}\right),} & \text { if } \rho(\mathbf{u})=\mathbf{v}, \\ {[2]_{\mathbf{v}}\left(\mathrm{c}_{\mathbf{u}}^{m, n+1}+\mathrm{c}_{\mathbf{u}}^{m+1, n-1}+\mathrm{c}_{\mathbf{u}}^{m-1, n}\right),} & \text { if } \rho^{-1}(\mathbf{u})=\mathbf{v},\end{cases}
$$


where again terms with negative indices are zero. Moreover, there are also the evident versions of (3-14) and (3-15) using ${ }^{m, n} \mathrm{v}$ instead of $\mathrm{c}_{\mathbf{v}}^{m, n}$.

Example 3.8. The reader should compare (3-14) and (3-15) with the recursion formulas from Lemma 2.7. This is no coincidence, keeping Remark 3.4 and Example 3.6 in mind. For example, one can easily check directly that

$$
\begin{gathered}
\mathrm{c}_{o}^{0,1} \theta_{g}=\left([2]_{\mathrm{v}}^{-1} \theta_{g} \theta_{o}\right) \theta_{g}=[2]_{\mathrm{v}}\left([2]_{\mathrm{v}}^{-2} \theta_{g} \theta_{o} \theta_{g}-\theta_{g}\right)+[2]_{\mathrm{v}} \theta_{g}=[2]_{\mathrm{v}}(\mathrm{c}_{g}^{1,1}+\underbrace{\mathrm{c}_{g}^{-1,1}}_{=0}+\mathrm{c}_{g}^{0,0}), \\
\theta_{g} \mathrm{c}_{o}^{0,1}=\theta_{g}\left([2]_{\mathrm{v}}^{-1} \theta_{g} \theta_{o}\right)=[3]_{\mathrm{v}} !\left([2]_{\mathrm{v}}^{-1} \theta_{g} \theta_{o}\right)=[3]_{\mathrm{v}} ! \mathrm{c}_{o}^{0,1},
\end{gathered}
$$

and similarly for right or left multiplication by $\theta_{0}$ or $\theta_{p}$.

Proposition 3.9. Each of the four sets

$$
\begin{aligned}
& \mathrm{H}^{\infty}=\{1\} \cup\left\{\mathrm{h}_{\mathbf{u}}^{k, l} \mid(k, l) \in X^{+}, \mathbf{u} \in G O P\right\}, \quad \mathrm{C}^{\infty}=\{1\} \cup\left\{\mathrm{c}_{\mathbf{u}}^{m, n} \mid(m, n) \in X^{+}, \mathbf{u} \in G O P\right\} \\
& { }^{\infty} \mathrm{H}=\{1\} \cup\left\{{ }_{\mathbf{u}} \mathrm{h} \mid(k, l) \in X^{+}, \mathbf{u} \in G O P\right\}, \quad{ }^{\infty} \mathrm{C}=\{1\} \cup\left\{\begin{array}{c}
m, n \\
\mathbf{u}
\end{array} \mid(m, n) \in X^{+}, \mathbf{u} \in G O P\right\}
\end{aligned}
$$

is a basis of $\mathrm{T}_{\infty}$.

As for Lemma 3.7, the proof of Proposition 3.9 is postponed until Section 4. As we will see, the bases $\mathrm{H}^{\infty}$ and ${ }^{\infty} \mathrm{H}$ could be called Bott-Samelson bases.

Following [KL79], we can define left, right and two-sided cells for $\mathrm{T}_{\infty}$. We have chosen to work with the basis $\mathrm{C}^{\infty}$.

Definition 3.10. We define a left pre-order on $\mathrm{C}^{\infty}$ by declaring $c_{\mathbf{u}}^{m, n} \geq_{\mathrm{L}} c_{\mathbf{v}}^{m^{\prime}, n^{\prime}}$ if there exists an element $\mathrm{Z} \in \mathrm{C}^{\infty}$ such that $\mathrm{c}_{\mathbf{v}}^{m, n}$ appears as a summand of $\mathrm{Zc}_{\mathbf{u}^{m^{\prime}}} n^{\prime}$, when the latter is written as a linear combination of elements in $\mathrm{C}^{\infty}$.

This pre-order gives rise to an equivalence relation by declaring $\mathrm{c}_{\mathbf{u}}^{m, n} \sim_{\mathrm{L}} \mathrm{c}_{\mathbf{v}}^{m^{\prime}, n^{\prime}}$ whenever $\mathrm{c}_{\mathbf{u}}^{m, n} \geq_{\mathrm{L}} c_{\mathbf{v}}^{m^{\prime}, n^{\prime}}$ and $c_{\mathbf{v}}^{m^{\prime}, n^{\prime}} \geq_{\mathrm{L}} c_{\mathbf{u}}^{m, n}$. The equivalence classes of $\sim_{\mathrm{L}}$ are called left cells.

Similarly, right multiplication gives rise to a right pre-order $\geq_{R}$, a right equivalence relation $\sim_{\mathrm{R}}$ and right cells R. Multiplication on both sides, gives rise to a two-sided pre-order $\geq_{\mathrm{J}}$, a two-sided equivalence relation $\sim_{\mathrm{J}}$ and two-sided cells $\mathrm{J}$.

Clearly, $1 \in \mathrm{T}_{\infty}$ forms a cell $\{1\}$ on its own, which is left, right and two-sided at once, and always the lowest cell. We call $\{1\}$ the trivial cell. The other cells are as follows.

Proposition 3.11. The non-trivial cells for the algebra $\mathrm{T}_{\infty}$ are

$$
\begin{gathered}
\mathrm{L}_{\mathbf{u}}=\left\{\mathrm{c}_{\mathbf{u}}^{m, n} \mid(m, n) \in X^{+}\right\}, \quad \mathbf{u}^{\mathrm{R}}=\left\{\begin{array}{l}
m, n \\
\mathbf{u}
\end{array} \mid(m, n) \in X^{+}\right\}, \quad \text { for } \mathbf{u} \in G O P, \\
\mathrm{~J}=\left\{\mathrm{c}_{\mathbf{u}}^{m, n} \mid(m, n) \in X^{+}, \mathbf{u} \in G O P\right\}=\left\{\begin{array}{l}
m, n \\
\mathbf{u}
\end{array} \mid(m, n) \in X^{+}, \mathbf{u} \in G O P\right\},
\end{gathered}
$$

where $L_{\mathbf{u}},{ }_{\mathbf{u}} R$ and $\mathrm{J}$ are left, right and two-sided cells respectively.

Proof. Fix $g$ as a starting color. Applying (3-15) to $\theta_{\mathbf{u}} c_{g}^{m, n}$, yields $\mathbf{c}_{g}^{m+1, n} \geq_{\mathbf{L}} \mathbf{c}_{g}^{m, n}$ for $\mathbf{u}$ being chosen such we can apply the middle cases. We also obtain $\mathrm{c}_{g}^{m, n} \geq_{\mathrm{L}} \mathrm{c}_{g}^{m+1, n}$, by applying (3-15) to $\theta_{\mathbf{v}} \mathbf{c}_{g}^{m+1, n}$ for appropriate $\mathbf{v}$. Thus, we have $\mathbf{c}_{g}^{m, n} \sim_{\mathrm{L}} \mathrm{c}_{g}^{m+1, n}$. Similarly, we deduce $\mathrm{c}_{g}^{m, n} \sim_{\mathrm{L}} \mathrm{c}_{g}^{m, n-1}, \mathrm{c}_{g}^{m, n} \sim_{\mathrm{L}} \mathrm{c}_{g}^{m, n+1}$ and $\mathrm{c}_{g}^{m, n} \sim_{\mathrm{L}} \mathrm{c}_{g}^{m-1, n}$. Thus, for fixed $m$ we get that all $\mathrm{c}_{g}^{m,}$ are in the same left cell, and similarly for fixed $n$ we get that all $\mathrm{c}_{g}^{, n}$ are in the same left cell. We can also deduce that $\mathrm{c}_{g}^{m, n} \sim_{\mathrm{L}} \mathrm{c}_{g}^{m-1, n+1}$ and $\mathrm{c}_{g}^{m, n} \sim_{\mathrm{L}} \mathrm{c}_{g}^{m+1, n-1}$. In summary, all $\mathrm{c}_{g}^{m, n}$ belong to the same left cell. Since left multiplication will never change the rightmost color of a word, we conclude that $\mathrm{L}_{g}$ is indeed a left cell.

Analogously, one can show that $\mathrm{L}_{o}$ and $\mathrm{L}_{p}$ are left cells, and, mutatis mutandis, that ${ }_{\mathbf{u}} \mathrm{R}$ is a right cell, for $\mathbf{u}$. Finally, the statement about two-sided cell follows from (3-14).

\section{C. The quotient of level $e$.}


Its definition. We are now ready to define interesting, finite-dimensional quotients of $\mathrm{T}_{\infty}$, which are compatible with the cell structure.

Definition 3.12. For fixed level $e$, let $\mathrm{I}_{e}$ be the two-sided ideal in $\mathrm{T}_{\infty}$ generated by

$$
\left\{\mathrm{c}_{\mathbf{u}}^{m, n} \mid m+n=e+1, \mathbf{u} \in G O P\right\}=\left\{{ }_{\mathbf{u}}^{m, n} \mid m+n=e+1, \mathbf{u} \in G O P\right\} .
$$

We define the the trihedral Hecke algebra of level $e$ as

$$
\mathrm{T}_{e}=\mathrm{T}_{\infty} / \mathrm{I}_{e}
$$

and we call $\mathrm{I}_{e}$ the vanishing ideal of level $e$.

Remark 3.13. We point out that $\mathrm{T}_{e}$ is the trihedral analog of the so called small quotient in the dihedral case, cf. 'The dihedral story' 3.24.

Proposition 3.14. Each of the two sets

$$
\begin{aligned}
& \mathrm{C}^{e}=\{1\} \cup\left\{\mathrm{c}_{\mathbf{u}}^{m, n} \mid 0 \leq m+n \leq e, \mathbf{u} \in G O P\right\}, \\
& { }^{e} \mathrm{C}=\{1\} \cup\left\{{ }^{m, n}{ }_{\mathbf{u}} \mathrm{c} \mid 0 \leq m+n \leq e, \mathbf{u} \in G O P\right\},
\end{aligned}
$$

is a basis of $\mathrm{T}_{e}$. Thus, we have $\operatorname{dim}_{\mathbb{C}_{\mathrm{v}}} \mathrm{T}_{e}=3 \frac{(e+1)(e+2)}{2}+1=3 t_{e}+1$.

Proof. By Lemma 3.7, $\mathrm{T}_{\infty}$ is an $\mathbb{N}^{0}$-filtered algebra with $\mathrm{T}_{\infty} \cong \bigcup_{i \in \mathbb{N}^{0}}\left(\mathrm{~T}_{\infty}\right)_{i}$ such that $\left(\mathrm{T}_{\infty}\right)_{0}=\{1\}$ and, for any $i \in \mathbb{Z}_{\geq 1}$, we have

$$
\left(\mathrm{T}_{\infty}\right)_{i}=\mathbb{C}_{\mathbf{v}}\left\{\mathrm{c}_{\mathbf{u}}^{m, n} \mid 0 \leq m+n \leq i-1, \mathbf{u} \in G O P\right\} .
$$

Note that $c_{\mathbf{u}}^{0,0}=\theta_{\mathbf{u}}$ has filtration degree 1 , so the multiplication rule in Lemma 3.7 is compatible with this filtration.

Since $\mathrm{I}_{e}$ is generated by homogeneous elements, $\mathrm{T}_{e}$ is also an $\mathbb{N}^{0}$-filtered algebra. In order to prove finite-dimensionality, consider the associated $\mathbb{N}^{0}$-graded algebra

$$
\mathcal{E}\left(\mathrm{T}_{e}\right)=\bigoplus_{i \in \mathbb{N}^{0}}\left(\mathrm{~T}_{e}\right)_{i} /\left(\mathrm{T}_{e}\right)_{i-1},
$$

where $\left(\mathrm{T}_{e}\right)_{-1}=\{0\}$, by convention. Note that

$$
\mathrm{c}_{\mathbf{u}}^{m, n} \equiv \mathrm{h}_{\mathbf{u}}^{m, n} \bmod \left(\mathrm{T}_{e}\right)_{m+n},
$$

for all $m, n$. We have $\mathcal{E}\left(\mathrm{T}_{e}\right)_{e+2}=\{0\}$, by Lemma 3.7, and $\mathcal{E}\left(\mathrm{T}_{e}\right)_{i}=\{0\}$ for all $i \geq e+3$, also by Lemma 3.7 .

The first statement follows, since $\left\{\mathrm{c}_{\mathbf{u}}^{m, n} \mid m+n=i-1, \mathbf{u} \in G O P\right\}$ is, by Proposition 3.9, a basis of $\mathcal{E}\left(\mathrm{T}_{e}\right)_{i}$, for all $1 \leq i \leq e+1$. The dimension formula is then clear.

The version with ${ }_{\mathbf{u}}^{m, n} \mathrm{c}$ can be shown verbatim.

Corollary 3.15. The non-trivial cells for the algebra $T_{e}$ are as in Proposition 3.11, but intersected with the bases from Proposition 3.14.

In particular, the non-trivial left and right cells have each cardinality $t_{e}=\frac{(e+1)(e+2)}{2}$. The non-trivial two-sided cell is the disjoint union of them all, so it has cardinality $3 t_{e}$.

Example 3.16. The left cells correspond to the generalized type A Dynkin diagrams $\mathbf{A}_{e}$ in Section AppA, which are cut-offs of the positive Weyl chamber as in (2-1), such that the basis elements of the left cells correspond to the vertices of the diagram.

The prototypical examples to keep in mind are

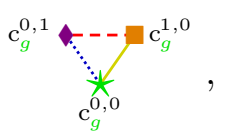

$\mathrm{L}^{g}$ for $e=1$

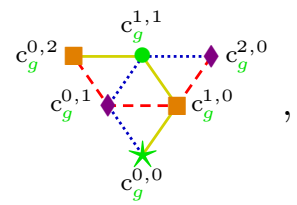

$\mathbf{L}^{g}$ for $e=2$

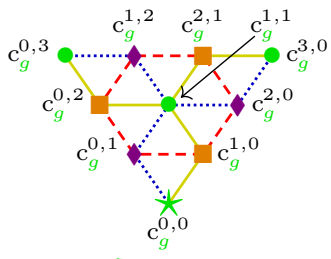

$\mathrm{L}^{g}$ for $e=3$ 
where we also display the associated colored KL basis elements. The starting (rightmost) color is indicated by $\star$. The color of any vertex is the color of the leftmost $\theta_{\mathbf{u}}$ in any of the terms of the corresponding colored KL basis element. The arrows of the same type, emanating from a given vertex, indicate the terms which appear on the right-hand side of the multiplication rule in (3-15).

Trihedral simples. Next, we classify all simple representations of $\mathrm{T}_{e}$ on $\left(\mathbb{C}_{\mathrm{v}^{-}}\right)$vector spaces, cf. (3-19). To this end, note that the ideal $\mathrm{I}_{e}$ defining $\mathrm{T}_{\infty}$ is built such that we can use Koornwinder's Chebyshev polynomials and their roots as in Section 2B below.

First, the one-dimensional representations of $\mathrm{T}_{e}$ are easy to define, since they correspond to characters. Each such character

$$
\mathrm{M}_{\lambda_{g}, \lambda_{o}, \lambda_{p}}: \mathrm{T}_{e} \rightarrow \mathbb{C}_{\mathrm{v}}
$$

is completely determined by its value on the generators

$$
\theta_{g} \mapsto \lambda_{g}, \theta_{o} \mapsto \lambda_{o}, \theta_{p} \mapsto \lambda_{p}
$$

Therefore, we can identify $\mathrm{M}_{\lambda_{g}, \lambda_{o}, \lambda_{p}}$ with a triple $\left(\lambda_{g}, \lambda_{o}, \lambda_{p}\right) \in \mathbb{C}_{\mathrm{v}}^{3}$.

Lemma 3.17. The following table

$$
\begin{array}{c:c}
e \equiv 0 \bmod 3 & e \not \equiv 0 \bmod 3 \\
\hline \mathrm{M}_{0,0,0}, \mathrm{M}_{[3]_{\mathrm{v}} !, 0,0}, & \mathrm{M}_{0,0,0} \\
\mathrm{M}_{0,[3]_{\mathrm{v}} !, 0}, \mathrm{M}_{0,0,[3]_{\mathrm{v}} !} &
\end{array}
$$

gives a complete, irredundant list of one-dimensional $\mathrm{T}_{e}$-representations.

Proof. Let us first check which triples $\left(\lambda_{g}, \lambda_{o}, \lambda_{p}\right)$ give a well-defined character of $\mathrm{T}_{e}$ : by (3-3), we see that $\lambda_{\mathbf{u}}$ has to be zero or [3] $]_{\mathbf{v}}$. Moreover, (3-4) implies that either all $\theta_{\mathbf{u}}$ act by zero, precisely one of them acts by $[3]_{\mathrm{v}}$ !, or all of them act by $[3]_{\mathrm{v}}$ !. Further restrictions are imposed by requiring the representation to vanish on $\mathrm{J}_{e}$.

Let us now give the details. The representation $\mathrm{M}_{0,0,0}$ vanishes on $\mathrm{J}_{e}$, because, by definition, $\mathrm{c}_{g}^{m, n}, \mathrm{c}_{o}^{m, n}, \mathrm{c}_{p}^{m, n}$ have no constant term for all $m, n$, since their starting color is always $\theta_{\mathbf{u}}$.

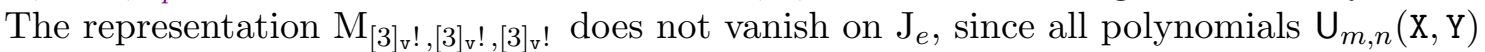
have a unique term of highest degree. This follows from the representation theory of of $\mathfrak{s l}_{3}$, since $\mathrm{X}^{m} \mathrm{Y}^{n}$ has a unique highest summand. This coefficient of this term contributes a maximal power of $\mathrm{v}$ when evaluated, which cannot be canceled by the coefficients of other terms, e.g.

$$
\mathrm{X}^{2}-\mathrm{Y} \leadsto[2]_{\mathrm{v}}^{-2} \theta_{p} \theta_{O} \theta_{g}-[2]_{\mathrm{v}}^{-1} \theta_{p} \theta_{g} \mapsto[2]_{\mathrm{v}}^{-2}[3]_{\mathrm{v}} ![3]_{\mathrm{v}} ![3]_{\mathrm{v}} !-[2]_{\mathrm{v}}^{-1}[3]_{\mathrm{v}} ![3]_{\mathrm{v}} ! \neq 0 .
$$

Thus, $\mathrm{M}_{[3]_{\mathrm{v}} !,[3]_{\mathrm{v}} !,[3]_{\mathrm{v}} !}$ is not a representation of $\mathrm{T}_{e}$.

When $e \equiv 0 \bmod 3$, there are three more characters, namely

$$
\mathrm{M}_{[3]_{\mathrm{v}} !, 0,0}, \quad \mathrm{M}_{0,[3]_{\mathrm{v}} !, 0}, \quad \mathrm{M}_{0,0,[3]_{\mathrm{v}} !} \cdot
$$

To see this note that, for $m+n=e+1$ and $e \equiv 0 \bmod 3$, we have $m+n \equiv 1 \bmod 3$. Hence, $m \equiv n \equiv 0,1 \bmod 3$ is impossible in this case. By Lemma 2.8, this means that $\mathrm{U}_{m, n}(\mathrm{X}, \mathrm{Y})$ does not have a non-zero constant term. It follows that all terms in $\mathrm{c}_{g}^{m, n}, \mathrm{c}_{o}^{m, n}, \mathrm{c}_{p}^{m, n}$ contain a factor $\theta_{\mathbf{v}} \theta_{\mathbf{u}}$ for some $\mathbf{u} \neq \mathbf{v}$. For any of the three $\mathrm{M}_{[3]_{\mathbf{v}} !, 0,0}, \mathrm{M}_{0,[3]_{\mathbf{v}} !, 0}$ or $\mathrm{M}_{0,0,[3]_{\mathbf{v}} !}$, we therefore have $\theta_{\mathbf{v}} \theta_{\mathbf{u}} \mapsto 0$. This shows that $\mathrm{c}_{g}^{m, n}, \mathrm{c}_{o}^{m, n}, \mathrm{c}_{p}^{m, n} \mapsto 0$.

The three corresponding one-dimensional representations are clearly non-isomorphic. 
Let us now study the simple representations of dimension three, which depend on a complex number $z \in \mathbb{C}$. To this end, we define three matrices

$$
\begin{gathered}
\mathrm{M}_{z}(g)=[2]_{\mathrm{v}}\left(\begin{array}{ccc}
{[3]_{\mathrm{v}}} & \bar{z} & z \\
0 & 0 & 0 \\
0 & 0 & 0
\end{array}\right), \quad \mathrm{M}_{z}(o)=[2]_{\mathrm{v}}\left(\begin{array}{ccc}
0 & 0 & 0 \\
z & {[3]_{\mathrm{v}}} & \bar{z} \\
0 & 0 & 0
\end{array}\right), \\
\mathrm{M}_{z}(p)=[2]_{\mathrm{v}}\left(\begin{array}{ccc}
0 & 0 & 0 \\
0 & 0 & 0 \\
\bar{z} & z & {[3]_{\mathrm{v}}}
\end{array}\right) .
\end{gathered}
$$

Let $\mathrm{M}_{z}^{\text {tot }}=\mathrm{M}_{z}(g)+\mathrm{M}_{z}(o)+\mathrm{M}_{z}(p)$.

Next, we use the explicit description of the elements in $\mathrm{V}_{e}$, cf. Remark 2.11.

Lemma 3.18. The matrices $\mathrm{M}_{z}(g), \mathrm{M}_{z}(o), \mathrm{M}_{z}(p)$ define a representation $\mathrm{M}_{z}$ of $\mathrm{T}_{e}$ on $\mathbb{C}_{\mathrm{v}}^{3}$, such that

$$
\theta_{g} \mapsto \mathrm{M}_{z}(g), \quad \theta_{o} \mapsto \mathrm{M}_{z}(o), \quad \theta_{p} \mapsto \mathrm{M}_{z}(p),
$$

if and only if $(z, \bar{z}) \in \mathrm{V}_{e}$.

Proof. Two short calculations show that $\mathrm{M}_{z}(\mathbf{u})$ respects the relations (3-3) and (3-4). The fact that $\mathrm{M}_{z}$ vanishes on $\mathrm{J}_{e}$ if and only if $(z, \bar{z}) \in \mathrm{V}_{e}$, follows by the proof of Lemma 2.10, as we defined $\mathrm{c}_{g}^{m, n}, \mathrm{c}_{o}^{m, n}$ and $\mathrm{c}_{p}^{m, n}$ in terms of $\mathrm{U}_{m, n}(\mathrm{X}, \mathrm{Y})$. Note that in the calculation of $\mathrm{M}_{z}\left(\mathrm{c}_{\mathbf{u}}^{m, n}\right)$ the positive powers of $[2]_{\mathrm{v}}$, due to (3-17), cancel against the negative powers of $[2]_{\mathrm{v}}$, which appear in (3-10), up to an overall factor $[2]_{\mathrm{v}}$.

Recall that $\zeta=\exp \left(2 \pi \mathrm{i} \frac{1}{3}\right)$.

Lemma 3.19. Let $(z, \bar{z}),\left(z^{\prime}, \bar{z}^{\prime}\right) \in \mathrm{V}_{e}, z \neq z^{\prime}$.

(3.19.a) $\mathrm{M}_{z} \cong \mathrm{M}_{z^{\prime}}$ as representations of $\mathrm{T}_{e}$ if and only if $z^{\prime}=\zeta^{ \pm 1} z$.

(3.19.b) $\mathrm{M}_{z}$ is simple if and only if $z \neq 0$.

Proof. (3.19.a). Suppose that $z^{\prime}=\zeta^{ \pm 1} z$. Then we have the following base change between $\mathrm{M}_{z}^{\text {tot }}$ and $\mathrm{M}_{z^{\prime}}^{\text {tot }}$ :

$$
[2]_{\mathrm{v}}\left(\begin{array}{ccc}
{[3]_{\mathrm{v}}} & \bar{z}^{\prime} & z^{\prime} \\
z^{\prime} & {[3]_{\mathrm{v}}} & \bar{z}^{\prime} \\
\bar{z}^{\prime} & z^{\prime} & {[3]_{\mathrm{v}}}
\end{array}\right)=[2]_{\mathrm{v}}\left(\begin{array}{ccc}
\zeta^{\mp 1} & 0 & 0 \\
0 & 1 & 0 \\
0 & 0 & \zeta^{ \pm 1}
\end{array}\right)\left(\begin{array}{ccc}
{[3]_{\mathrm{v}}} & \bar{z} & z \\
z & {[3]_{\mathrm{v}}} & \bar{z} \\
\bar{z} & z & {[3]_{\mathrm{v}}}
\end{array}\right)\left(\begin{array}{ccc}
\zeta^{ \pm 1} & 0 & 0 \\
0 & 1 & 0 \\
0 & 0 & \zeta^{\mp 1}
\end{array}\right)
$$

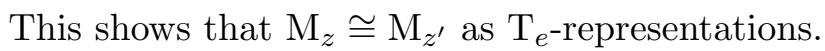

To see the converse, we compute the eigenvalues and eigenvectors of $\mathrm{M}_{z}^{\text {tot }}$ :

$$
\begin{array}{c:c:c}
{[2]_{\mathrm{v}}\left(z+\bar{z}+[3]_{\mathrm{v}}\right)} & {[2]_{\mathrm{v}}\left(\zeta^{-1} z+\zeta \bar{z}+[3]_{\mathrm{v}}\right)} & {[2]_{\mathrm{v}}\left(\zeta z+\zeta^{-1} \bar{z}+[3]_{\mathrm{v}}\right)} \\
(1,1,1) \in \mathbb{C}_{\mathrm{v}}^{3} & \left(1, \zeta, \zeta^{-1}\right) \in \mathbb{C}_{\mathrm{v}}^{3} & \left(1, \zeta^{-1}, \zeta\right) \in \mathbb{C}_{\mathrm{v}}^{3}
\end{array}
$$

Since $\mathrm{v}$ is generic, these are three non-zero eigenvalues with three linearly independent eigenvectors showing that $\mathrm{M}_{z}^{\text {tot }}$ can be diagonalized.

Now suppose $\mathrm{M}_{z} \cong \mathrm{M}_{z^{\prime}}$. Then $\mathrm{M}_{z}^{\text {tot }}$ and $\mathrm{M}_{z^{\prime}}^{\text {tot }}$ must have the same eigenvalues, so the above implies that one of the following three triples of equations must hold.

$$
\begin{aligned}
& z+\bar{z}=z^{\prime}+\bar{z}^{\prime} \quad \text { and } \quad \zeta z+\zeta^{-1} \bar{z}=\zeta z^{\prime}+\zeta^{-1} \bar{z}^{\prime} \quad \text { and } \quad \zeta^{-1} z+\zeta \bar{z}=\zeta^{-1} z^{\prime}+\zeta \bar{z}^{\prime}, \\
& z+\bar{z}=\zeta z^{\prime}+\zeta^{-1} \bar{z}^{\prime} \text { and } \zeta z+\zeta^{-1} \bar{z}=\zeta^{-1} z^{\prime}+\zeta \bar{z}^{\prime} \text { and } \zeta^{-1} z+\zeta \bar{z}=z^{\prime}+\bar{z}^{\prime}, \\
& z+\bar{z}=\zeta^{-1} z^{\prime}+\zeta \bar{z}^{\prime} \quad \text { and } \quad \zeta z+\zeta^{-1} \bar{z}=z^{\prime}+\bar{z}^{\prime} \text { and } \zeta^{-1} z+\zeta \bar{z}=\zeta z^{\prime}+\zeta^{-1} \bar{z}^{\prime} .
\end{aligned}
$$

One easily checks that these are satisfied if and only if $z=z^{\prime}$ (top triple), $z=\zeta z^{\prime}$ (middle triple) or $z=\zeta^{-1} z^{\prime}$ (bottom triple). 
(3.19.b). In case $z=0$, one clearly has

$$
\mathrm{M}_{0} \cong \mathrm{M}_{[3]_{\mathrm{v}} !, 0,0} \oplus \mathrm{M}_{0,[3]_{\mathrm{v}} !, 0} \oplus \mathrm{M}_{0,0,[3]_{\mathrm{v}} !},
$$

where the one-dimensional representations were defined in (3-16).

Now suppose that $z \neq 0$ and that $\mathrm{M}_{z}$ is reducible. Then it must have a subrepresentation of dimension one or two. The explicit description of the eigenvalues and eigenvectors of $\mathrm{M}_{z}^{\text {tot }}$ from (3-18) shows that this is impossible.

To see this, first note that the restriction of $\mathrm{M}_{z}^{\text {tot }}$ to the vector space underlying the potential subrepresentation would be diagonalizable as well.

Secondly, in case the eigenvalues in (3-18) are all distinct, at least one eigenvector therein is also an eigenvector for the restriction. However, applying $\mathbf{M}_{z}(g), \mathbf{M}_{z}(o)$ and $\mathbf{M}_{z}(p)$ to any of the three eigenvectors in (3-18) gives three linear independent vectors, which shows that no subrepresentation can exist in case of distinct eigenvalues.

Thirdly, assume that two of the three eigenvalues in (3-18) coincide. Then there must exist a linear combination of the corresponding two eigenvectors which is an eigenvector for the restriction. Applying $\mathbf{M}_{z}(g), \mathbf{M}_{z}(o)$ and $\mathbf{M}_{z}(p)$ to that eigenvector would give three linear independent vectors, as can easily be checked. We get a contradiction again.

Finally, since $z \neq 0$, not all eigenvalues in (3-18) can be equal, so we are done.

If $e \not \equiv 0 \bmod 3$, then $Z(\sigma, \tau) \neq 0$ for all $(\sigma, \tau)$ as in (2-11). By Lemma 3.19, this implies that the total number of pairwise non-isomorphic $\mathrm{M}_{z}$ is equal to $\frac{t_{e}}{3}$. If $e \equiv 0 \bmod 3$, then that number is equal to $\frac{\left(t_{e}-1\right)}{3}$, because $Z(\sigma, \tau)=0$ if and only if $k=l=\frac{e}{3}$.

Summarized, we have the following non-isomorphic, simple $\mathrm{T}_{e}$-representations:

\begin{tabular}{r|c|c} 
& $e \equiv 0 \bmod 3$ & $e \not \equiv 0 \bmod 3$ \\
\hline one-dim. & $\mathrm{M}_{0,0,0}, \mathrm{M}_{[3]_{\mathrm{v}}, 0,0}$, & $\mathrm{M}_{0,0,0}$ \\
& $\mathrm{M}_{0,[3]_{\mathrm{v}} !, 0}, \mathrm{M}_{0,0,[3]_{\mathrm{v}} !}$ & 1 \\
quantity & 4 & 1 \\
\hline three-dim. & $\mathrm{M}_{z},(z, \bar{z}) \in \mathrm{V}_{e}^{\zeta}-\{(0,0)\}$ & $\mathrm{M}_{z},(z, \bar{z}) \in \mathrm{V}_{e}^{\zeta}$ \\
quantity & $\frac{\left(t_{e}-1\right)}{3}$ & $\frac{t_{e}}{3}$
\end{tabular}

Here $\mathrm{V}_{e}^{\zeta}$ denotes the set of $\mathbb{Z} / 3 \mathbb{Z}$-orbits in $\mathrm{V}_{e}$ under the action $(z, \bar{z}) \mapsto\left(\zeta z, \zeta^{-1} \bar{z}\right)$.

Example 3.20. By (3-19), the three-dimensional simple representation of $T_{e}$ are indexed by the $\mathbb{Z} / 3 \mathbb{Z}$-orbits of points in the interior of $d_{3}$ (cf. Example 2.12.), e.g.:

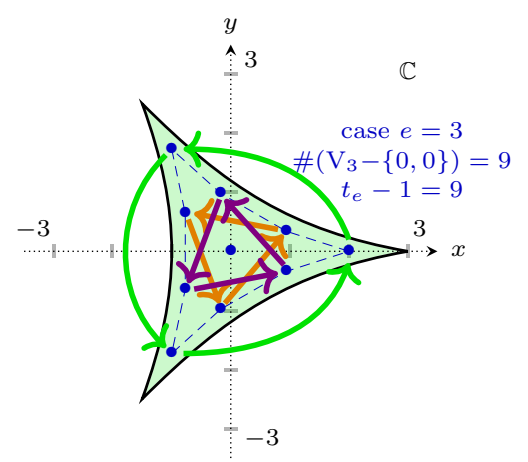

Here the arrows indicate the $\mathbb{Z} / 3 \mathbb{Z}$-symmetry.

We are now ready to provide a classification of simple $\mathrm{T}_{e}$-representations. 
Theorem 3.21. The table (3-19) gives a complete, irredundant list of simple $\mathrm{T}_{e}$-representations. Furthermore, the algebra $\mathrm{T}_{e}$ is semisimple.

Proof. By Proposition 3.14 the algebra $\mathrm{T}_{e}$ is of dimension $3 \frac{(e+1)(e+2)}{2}+1$. From the representation theory of finite-dimensional algebras we thus have

$$
\operatorname{dim}_{\mathbb{C}_{\mathrm{v}}} \mathrm{T}_{e}=3 \frac{(e+1)(e+2)}{2}+1=3 t_{e}+1 \geq \sum_{\mathrm{M}}\left(\operatorname{dim}_{\mathbb{C}_{\mathrm{v}}} \mathrm{M}\right)^{2}
$$

where the sum is taken over any set of pairwise non-isomorphic, simple $\mathrm{T}_{e}$-representations $\mathrm{M}$. If equality holds in (3-20) for such a set, then that set is complete and $\mathrm{T}_{e}$ is semisimple.

Case $e \not \equiv 0$ mod 3 . We use the data from (3-19) in (3-20) and obtain

$$
\sum_{\mathrm{M}}\left(\operatorname{dim}_{\mathbb{C}_{\mathrm{v}}} \mathrm{M}\right)^{2}=\frac{t_{e}}{3} \cdot 3^{2}+1 \cdot 1^{2}=3 t_{e}+1,
$$

which shows both statements.

Case $e \equiv 0 \bmod 3$. Similarly, we compute

$$
\sum_{\mathrm{M}}\left(\operatorname{dim}_{\mathbb{C}_{\mathrm{v}}} \mathrm{M}\right)^{2}=\frac{\left(t_{e}-1\right)}{3} \cdot 3^{2}+4 \cdot 1^{2}=3\left(t_{e}-1\right)+4=3 t_{e}+1,
$$

which again shows both statements.

3D. Generalizing dihedral Hecke algebras. We finish this section by listing some analogies to the dihedral case. The crucial link between the dihedral and the trihedral case is the following: The $\mathfrak{s l}_{2}$-version of the polynomial $\mathrm{U}_{m, n}(\mathrm{X}, \mathrm{Y})$ from Section $2 \mathrm{~B}$ is the Chebyshev polynomial $\mathrm{U}_{m}(\mathrm{X})$ (normalized and of the second kind). Using the convention that the $\mathrm{U}_{m}(\mathrm{X})$ are zero for negative subscripts, they satisfy the recursion relation

$$
\mathrm{U}_{0}(\mathrm{X})=1, \quad \mathrm{U}_{1}(\mathrm{X})=\mathrm{X}, \quad \mathrm{XU}_{m}(\mathrm{x})=\mathrm{U}_{m+1}(\mathrm{X})+\mathrm{U}_{m * 1}(\mathrm{X}) .
$$

Here, $\mathrm{X}$ corresponds to the fundamental representation of $\mathfrak{s l}_{2}$. The analog of the discoid $\mathrm{d}_{3}$ from (2-9) is the interval $d_{2}=[-2,2]$, whose boundary is the pair of primitive, complex second roots of unity, multiplied by 2 . (Note the evident $\mathbb{Z} / 2 \mathbb{Z}$-symmetry of $d_{2}$.)

The dihedral story 3.22. Let $\mathrm{D}_{\infty}=\mathrm{H}_{\mathrm{v}}\left(\mathrm{I}_{2}(\infty)\right)=\mathrm{H}_{\mathrm{v}}\left(\tilde{\mathrm{A}}_{1}\right)$ denote the dihedral Hecke algebra of the infinite dihedral group, i.e. the Weyl group of affine type $\mathrm{A}_{1}$, and $\mathrm{H}_{\mathrm{v}}\left(\mathrm{I}_{2}(e+2)\right)$ the dihedral Hecke algebra of dimension $2(e+2)$, which is of finite Coxeter type $\mathrm{I}_{2}(e+2)$. The first analogy of our story to the dihedral case is provided by Lemma 3.2, the difference being that the trihedral Hecke algebra is a proper subalgebra of $\mathrm{H}_{\mathrm{v}}$. The entries of the change-of-basis matrix from the (colored) KL basis to the Bott-Samelson basis of $\mathrm{D}_{\infty}$ are precisely the coefficients of the polynomials $\mathrm{U}_{m}(\mathrm{X})$, see for example [Eli16, Section 2.2].

The dihedral story 3.23. By Proposition 3.11, all non-trivial cells of $\mathrm{T}_{\infty}$ are infinite, and there are three non-trivial left and right cells, one for each $\mathbf{u} \in G O P$, whose disjoint union forms the only non-trivial two-sided cell. This is another analogy to the dihedral case: the algebra $\mathrm{D}_{\infty}$ has two non-trivial left and right cells, one for each of its Coxeter generators, whose disjoint union forms the only non-trivial two-sided cell.

The dihedral story 3.24. Let $\mathrm{D}_{e}$ denote the small quotient of $\mathrm{H}_{\mathrm{v}}\left(\mathrm{I}_{2}(e+2)\right)$, obtained by killing the top cell. Section $3 \mathrm{C}$ provides the third analogy: $\mathrm{D}_{e}$ can be obtained as a quotient of $\mathrm{D}_{\infty}$ by the ideal generated by the two elements related to the irreducible $\mathfrak{s l}_{2}$-module $\mathrm{L}_{e+1}$ under the quantum Satake correspondence; the non-trivial left cells of $\mathrm{D}_{e}$ have order $e+1$ and $\operatorname{dim}_{\mathbb{C}_{\mathrm{v}}} \mathrm{D}_{e}=2(e+2)-1=2 e+3$.

The dihedral story $\mathbf{3 . 2 5}$. Theorem 3.21 provides another analogy to the dihedral case: $\mathrm{D}_{e}$ is semisimple over $\mathbb{C}$, and all of its simples are either one- or two-dimensional, with the number of their isomorphism classes depending on whether $e \equiv 0 \bmod 2$ or $e \equiv 1 \bmod 2$. Analogously to (3-17), the two-dimensional simples can be defined by matrices whose off-diagonal, non-zero 
entries are the roots of the Chebyshev polynomials $\mathrm{U}_{e+1}(\mathrm{x})$, i.e. its (colored) KL generators are send to $\left(\begin{array}{ccc}{\left[\begin{array}{c}{[]_{v}} \\ 0\end{array}\right.} & 0\end{array}\right)$ and $\left(\begin{array}{cc}0 & 0 \\ z & {[2]_{v}}\end{array}\right)$, where $z=\bar{z}$ is a root of $\mathrm{U}_{e+1}(\mathrm{X})$.

\section{Trihedral Soergel Bimodules}

The purpose of this section is to categorify the trihedral Hecke algebras $T_{\infty}$ and $T_{e}$ from Section 3, where $e$ still denotes the level. As before, we have collected some analogies to the dihedral case at the end of the section, cf. Section 4D

4A. Bott-Samelson bimodules for affine $A_{2}$. First, we recall the diagrammatic 2category $\boldsymbol{s} \mathscr{B} \mathscr{S}_{\text {[q] }}$ from [Eli17, Section 3.3]. We call it the (2-category of) singular BottSamelson bimodules of affine type $\mathrm{A}_{2}$.

2-categorical conventions. For generalities and terminology on 2-categories, we refer for example to [Lei98] or [ML98].

Convention 4.1. We use 2-categories given by generators and relations. This means that 1-morphisms are obtained by compositions $\circ$ of the generating 1-morphisms, and 2-morphisms are obtained by horizontal $\circ_{h}$ and vertical $\circ_{v}$ compositions of the 2-generators whenever this makes sense. (In particular, the interchange law leads to additional relations in our 2-categories, called height relations.) Relations are supposed to hold between 2-morphisms. Details about such 2-categories can be found e.g. in [Rou08, Section 2.2].

Convention 4.2. We read 1-morphisms from right to left, using the operator-notation, and 2 -morphisms from bottom to top and right to left. These conventions are illustrated in Definition 4.6 below. Note that we usually omit the 1-morphisms in the pictures, and we will simplify diagrams by drawing them in a more topological fashion, using e.g. Example 4.8.

Convention 4.3. A (ZZ-)graded 2-category for us is a 2-category whose 2-hom spaces are $(\mathbb{Z}$-)graded, meaning that the 2-generators have a given degree, the relations are homogeneous and the degree is additive under horizontal and vertical composition. Moreover, 1-morphisms are formal shifts of generating 1-morphisms, indicated by $\{a\}$ for $a \in \mathbb{Z}$, so there is a formal $\mathbb{Z}$-action on 1-morphisms such that $\{k\}(\mathrm{F}\{a\})=\mathrm{F}\{a+k\}$ for all $k \in \mathbb{Z}$. Finally, a 2-morphism $\mathrm{f}: \mathrm{F}\{0\} \rightarrow \mathrm{G}\{0\}$, homogeneous of degree $d$, is of degree $d-a+b$ seen as a 2-morphism $f: F\{a\} \rightarrow G\{b\}$. For more information on such 2-categories, see e.g. [Lau10, Section 5.1].

The definition of $\boldsymbol{s} \mathscr{B} \mathscr{S}_{[\mathrm{q}]}$. Let $\mathbb{C}_{[\mathrm{q}]}=\mathbb{C}\left[\mathrm{q}, \mathrm{q}^{-1}\right]$ and $\mathrm{R}_{[\mathrm{q}]}=\mathbb{C}_{[\mathrm{q}]}\left[\alpha_{b}, \alpha_{r}, \alpha_{y}\right]$, where $\alpha_{b}, \alpha_{r}, \alpha_{y}$ are formal variables. We define an action of the affine Weyl group $\mathrm{W}$ from Section $3 \mathrm{~B}$ on $\mathrm{R}_{[\mathrm{q}]}$ :

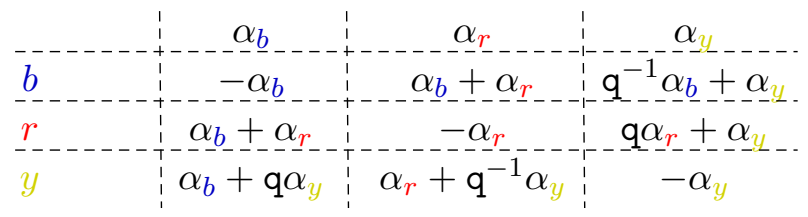

One easily checks that (4-1) is well-defined. This also gives rise to an action of the secondary colors on $\mathrm{R}_{[\mathrm{q}]}$ by using (3-2) (recalling that e.g. $g=\{b, y\}$ ). Thus, we can define:

Definition 4.4. For any $c \in B R Y$ and $\mathbf{u} \in G O P$, let $\mathbf{R}_{[\mathrm{q}]}^{c}$ and $\mathbf{R}_{[q]}^{\mathbf{u}}$ denote the subrings of $\mathrm{R}_{[q]}$ consisting of all $c$-invariant and $\mathbf{u}$-invariant elements, respectively.

Recall that we always use $\mathbf{u}, \mathbf{v} \in G O P$ as secondary dummy colors, and we also use the primary dummy colors $c, d \in B R Y$ from now on. Moreover, identifying our colors with proper subsets of $B R Y$, including the empty subset, we say that two of them are compatible 
if one is a subset of the other, e.g. as the colors connected by an edge below.
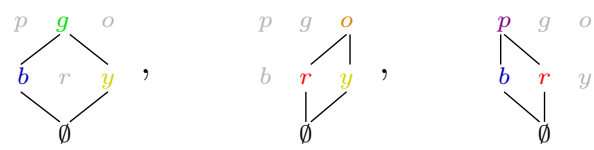

Example 4.5. The color $b$ is compatible with $\emptyset, g$ and $p$, but not with $r, y$ or $o$.

We will define the 2-category of singular Soergel bimodules as a quotient of the following 2 -category, which we view as a free version of it.

Definition 4.6. Let $\boldsymbol{s} \mathscr{B} \mathscr{S}_{[\mathrm{q}]}^{*}$ be the 2-category defined as follows.

'Objects of $\boldsymbol{s} \mathscr{B} \mathscr{S}_{[\mathrm{q}]}^{*}$ '. The objects are proper subsets of $B R Y=\{b, y, r\}$, including the empty subset $\emptyset$. The one-element subsets are identified with $b, y, r$, the two-element subsets are identified with $g, o, p$, using the color conventions from Section $3 \mathrm{~A}$.

'1-morphisms of $\boldsymbol{s} \mathscr{B} \mathscr{S}_{[\mathrm{qq}}^{*}$ '. By definition, there is one generating 1-morphism for each pair of distinct compatible colors. Namely, including all other compatible variations using the conventions from (4-2) and writing e.g. $b \emptyset=b \circ \emptyset$ for short:

$$
\emptyset b: \emptyset \leftarrow b, \quad b \emptyset: b \leftarrow \emptyset, \quad b g: b \leftarrow g, \quad y g: y \leftarrow g, \quad g b: g \leftarrow b, \quad g y: g \leftarrow y, \quad \text { etc. }
$$

'2-morphisms of $\boldsymbol{s} \mathscr{B} \mathscr{S}_{[\mathrm{q}]}^{*}$ '. The 2-morphisms are generated by two kinds of 2-generators. The first kind are cups, caps and crossings given as follows.
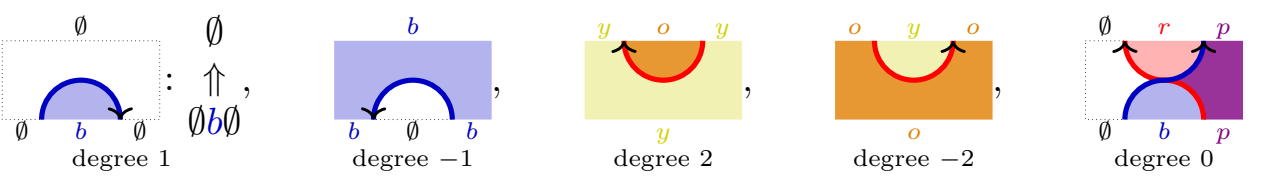

(We frame $\emptyset$-colored regions for readability.) The generators displayed in (4-3) are all generators up to colored variations: each strand separates two regions colored by subsets of $B R Y$ that differ by a primary color, which is used to color that strand. The strands are oriented such that the region colored by the smaller subset of $B R Y$ lies to their left.

The second kind of 2-generators are decorations of the regions by polynomials in $\mathrm{R}_{[\mathrm{q}]}$ that are invariant under the parabolic subgroup corresponding to the color of the region, i.e.

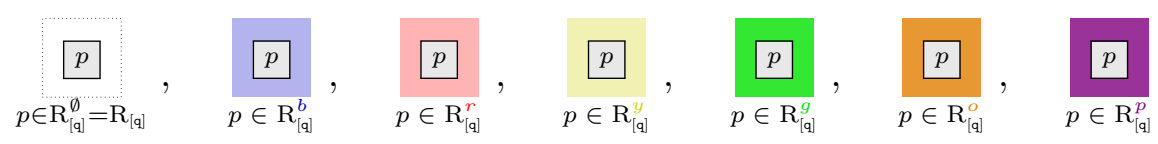

The polynomials are allowed to move around as long as they do not cross any strand.

'Grading on $\boldsymbol{s} \mathscr{B} \mathscr{S}_{[\mathrm{q}]}^{*}$ '. We endow $\boldsymbol{s} \mathscr{B} \mathscr{S}_{\text {[q] }}^{*}$ with the structure of a graded 2-category by giving the generators from (4-3) and (4-4) the following degree.

- Clockwise cups and caps between $\emptyset$ and $c$ have degree 1 , while their anticlockwise counterparts have degree -1 .

- Clockwise cups and caps between $c$ and a compatible $\mathbf{u}$ have degree 2, while their anticlockwise counterparts have degree -2 .

- Crossings are of degree 0 .

- Homogeneous polynomials are graded by twice their polynomial degree, i.e. the formal variables $\alpha_{b}, \alpha_{r}, \alpha_{y}$ are of degree 2 .

We have indicate some of these in (4-3). 
Example 4.7. In general, a 1-morphism is a finite string of generating 1-morphisms, which are indicated by their source and target, e.g. yorpb $\emptyset: y \leftarrow o \leftarrow r \leftarrow p \leftarrow b \leftarrow \emptyset$. (By convention, we identify the objects $c, \mathbf{u}$ with the identity 1-morphisms on them.) Furthermore,

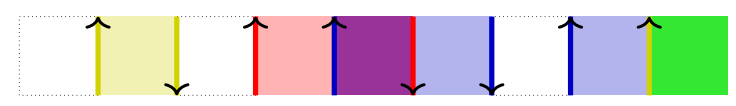

is an example of the coloring of facets and strands.

Example 4.8. As usual, one can define sideways crossings, e.g.
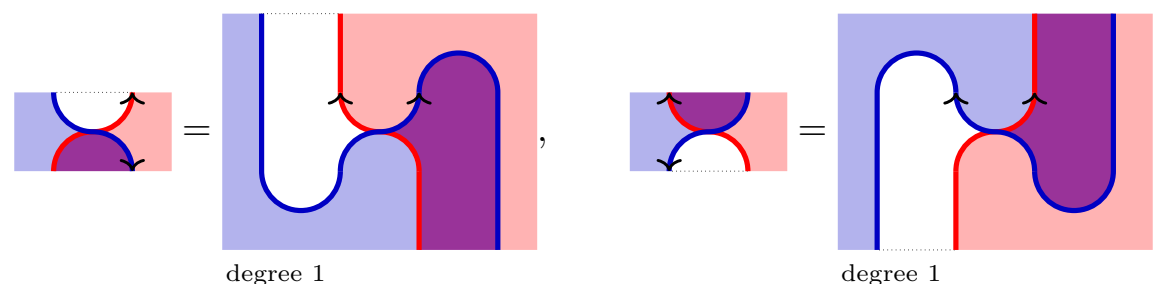

Note that these are of degree 1.

Remark 4.9. The 2-category $\boldsymbol{s} \mathscr{B} \mathscr{S}_{\text {[q] }}^{*}$ depends on q, since the quantum parameter is in the definition of the rings $\mathrm{R}_{[\mathrm{q}]}$, cf. (4-1).

Before we can go on, we need some algebraic notions.

An interlude on Frobenius extensions. The relations of $\boldsymbol{s} \mathscr{B} \mathscr{S}_{[\mathrm{q}]}$ actually come from a cube of Frobenius extensions. (For details on Frobenius extensions see e.g. [ESW17].)

Definition 4.10. A (commutative) Frobenius extension is an extension of commutative rings $\mathrm{R}^{\prime} \subset \mathrm{R}$ with $\mathrm{R}$ being a free $\mathrm{R}^{\prime}$-bimodule of finite rank, together with a $\mathrm{R}^{\prime}$-bilinear trace map $\partial: R \rightarrow R^{\prime}$ which gives rise to a non-degenerate bilinear pairing

$$
\langle\cdot, \cdot\rangle: \mathrm{B} \times \mathrm{B}^{\star} \rightarrow \mathrm{R}^{\prime} .
$$

Moreover, for a Frobenius extension there exist two $\mathrm{R}^{\prime}$-bases $\mathrm{B}, \mathrm{B}^{\star}$ of $\mathrm{R}$, such that for any $\mathrm{x} \in \mathrm{B}$ there is precisely one element $\mathrm{x}^{\star} \in \mathrm{B}^{\star}$ satisfying

$$
\left\langle\mathrm{x}, \mathrm{x}^{\prime}\right\rangle=\partial\left(\mathrm{xx}^{\prime}\right)=\delta_{\mathrm{x}^{\prime}, \mathrm{x}^{\star}} .
$$

The elements $\mathrm{x}$ and $\mathrm{x}^{\star}$, respectively the bases $\mathrm{B}$ and $\mathrm{B}^{\star}$ are called dual to each other.

The number of elements $\# \mathrm{~B}=\# \mathrm{~B}^{\star}$ is called the rank.

Such an extension is called graded if $R, R^{\prime}$ are graded rings, $R$ is graded as an $R^{\prime}$-bimodule, $\mathrm{B}, \mathrm{B}^{\star}$ consist of homogeneous elements, and $\partial$ is a homogeneous map.

Note that the dual elements $\mathrm{x}, \mathrm{x}^{\star}$ satisfy $\operatorname{deg}(\mathrm{x})+\operatorname{deg}\left(\mathrm{x}^{\star}\right)=-\operatorname{deg}(\partial)$.

Definition 4.11. We let $\partial_{c}: \mathrm{R}_{[\mathrm{q}]} \rightarrow \mathrm{R}_{[\mathrm{q}]}^{c}$ be defined via the formula $\partial_{c}(f)=\frac{f-c(f)}{\alpha_{c}}$. We call these the primary Demazure operators. Similarly, we define

$$
\begin{array}{ccc}
\partial_{g}^{b}=\mathrm{q} \partial_{b} \partial_{y}: \mathrm{R}_{[\mathrm{q}]}^{b} \rightarrow \mathrm{R}_{[\mathrm{qq}]}^{g}, & \partial_{g}^{y}=\partial_{y} \partial_{b}: \mathrm{R}_{[\mathrm{qq}}^{y} \rightarrow \mathrm{R}_{[\mathrm{q}]}^{g}, & \partial_{o}^{r}=\mathrm{q}^{-1} \partial_{r} \partial_{y}: \mathrm{R}_{[\mathrm{q}]}^{r} \rightarrow \mathrm{R}_{[\mathrm{q}]}^{o}, \\
\partial_{o}^{y}=\partial_{y} \partial_{r}: \mathrm{R}_{[\mathrm{q}]}^{y} \rightarrow \mathrm{R}_{[\mathrm{q}]}^{o}, & \partial_{p}^{b}=\partial_{b} \partial_{r}: \mathrm{R}_{[\mathrm{q}]}^{b} \rightarrow \mathrm{R}_{[\mathrm{q}]}^{p}, & \partial_{p}^{r}=\partial_{r} \partial_{b}: \mathrm{R}_{[\mathrm{q}]}^{r} \rightarrow \mathrm{R}_{[\mathrm{q}]}^{p},
\end{array}
$$

which we call the mixed Demazure operators. Finally, we define

$$
\begin{gathered}
\partial_{g}=\mathrm{q} \partial_{b} \partial_{y} \partial_{b}=\partial_{y} \partial_{b} \partial_{y}: \mathrm{R}_{[\mathrm{q}]} \rightarrow \mathrm{R}_{[\mathrm{q}}^{g}, \quad \partial_{o}=\mathrm{q}^{-1} \partial_{r} \partial_{y} \partial_{r}=\partial_{y} \partial_{r} \partial_{y}: \mathrm{R}_{[\mathrm{q}]} \rightarrow \mathrm{R}_{[\mathrm{q}]}^{o}, \\
\partial_{p}=\partial_{b} \partial_{r} \partial_{b}=\partial_{r} \partial_{b} \partial_{r}: \mathrm{R}_{[\mathrm{q}]} \rightarrow \mathrm{R}_{[\mathrm{q}]}^{p},
\end{gathered}
$$

which we call the primary Demazure operators. 
Note that the action on the linear terms determines the whole action since we have the twisted Leibniz rule $\partial_{c}(f g)=\partial_{c}(f) g+c(f) \partial_{c}(g)$. Moreover, a straightforward calculation (cf. [Eli17, (3.9)]) yields

$$
\mathrm{q} \partial_{b} \partial_{y} \partial_{b}=\partial_{y} \partial_{b} \partial_{y}, \quad \mathrm{q}^{-1} \partial_{r} \partial_{y} \partial_{r}=\partial_{y} \partial_{r} \partial_{y}, \quad \partial_{b} \partial_{r} \partial_{b}=\partial_{r} \partial_{b} \partial_{r},
$$

showing that the mixed Demazure operators are well-defined. (The careful reader might additionally want to check that the primary Demazure operators are well-defined by checking that $\partial_{c}(f)$ is a $c$-invariant polynomial.)

Remark 4.12. Recalling that the root variables are of degree 2, one easily observes that the primary, mixed and secondary Demazure operators are homogeneous of degree $-2,-4$ and -6 , respectively.

Lemma 4.13. We have Frobenius extensions

$$
\partial_{c}: \mathrm{R}_{[\mathrm{q}]} \rightarrow \mathrm{R}_{[\mathrm{q}]}^{c}, \quad \partial_{\mathbf{u}}^{c}: \mathrm{R}_{[\mathrm{q}]}^{c} \rightarrow \mathrm{R}_{[\mathrm{q}]}^{\mathrm{u}}, \quad \partial_{\mathbf{u}}: \mathrm{R}_{[\mathrm{q}]} \rightarrow \mathrm{R}_{[\mathrm{q}]}^{\mathrm{u}},
$$

of rank 2,3 and 6, respectively, which are compatible in the sense that $\partial_{c}=\partial_{\mathbf{u}}^{c} \partial_{\mathbf{u}}$.

Proof. One can prove this lemma by computing explicit dual bases. (Note that this requires 2 and 3 to be invertible.) We do not need them here and omit the calculations.

Definition 4.14. Choose any pairs of dual bases $\mathrm{B}_{c}, \mathrm{~B}_{c}^{\star}$ of $\partial_{c}: \mathrm{R}_{[\mathrm{q}]} \rightarrow \mathrm{R}_{[\mathrm{q}]}^{c}, \mathrm{~B}_{\mathbf{u}}^{c},\left(\mathrm{~B}_{\mathbf{u}}^{c}\right)^{\star}$ of $\partial_{\mathbf{u}}^{c}: \mathrm{R}_{[\mathrm{q}]}^{c} \rightarrow \mathrm{R}_{[\mathrm{q}]}^{\mathbf{u}}$ and $\mathrm{B}_{\mathbf{u}}, \mathrm{B}_{\mathbf{u}}^{\star}$ of $\partial^{\mathbf{u}}: \mathrm{R}_{[\mathrm{q}]} \rightarrow \mathrm{R}_{[\mathrm{q}]}^{\mathbf{u}}$. Let

$$
\Delta_{c}=\sum_{a \in \mathrm{B}_{c}} a \otimes a^{\star}, \quad \Delta_{\mathbf{u}}^{c}=\sum_{a \in \mathrm{B}_{\mathbf{u}}^{c}} a \otimes a^{\star}, \quad \Delta_{\mathbf{u}}=\sum_{a \in \mathrm{B}_{\mathbf{u}}} a \otimes a^{\star},
$$

where $a^{\star}$ denotes the basis element dual to $a$.

Note that the elements $\Delta_{-}^{-}$are well-defined, i.e. do not depend on the choice of dual bases (see [Eli17, Section 2.4]).

Definition 4.15. We define the following elements $\mu_{-}^{-}$in $\mathrm{R}_{[\mathrm{q}]}$.

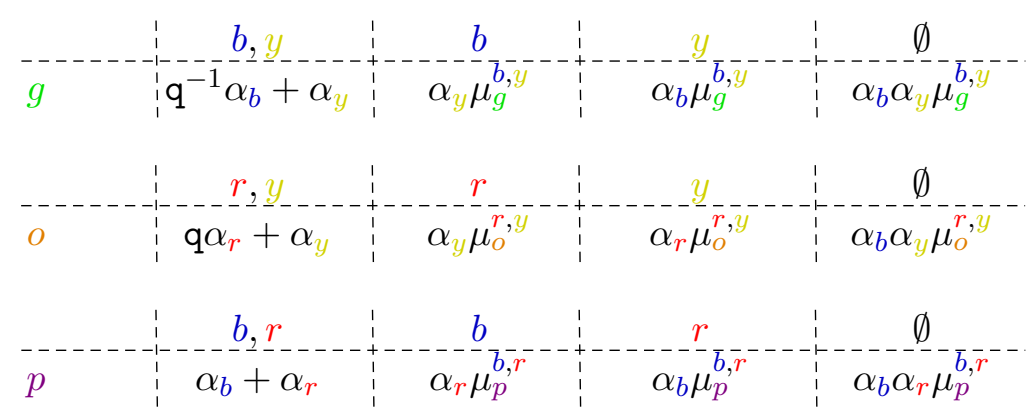

This is to be read as e.g. $\mu_{g}^{b, y}=\mathrm{q}^{-1} \alpha_{b}+\alpha_{y}$ and $\mu_{g}^{\emptyset}=\mu_{g}=\alpha_{b} \alpha_{y}\left(\mathbf{q}^{-1} \alpha_{b}+\alpha_{y}\right)$.

The continuation of definition of $\boldsymbol{s} \mathscr{B} \mathscr{S}_{[\mathrm{q}]}$.

Definition 4.16. Let $\boldsymbol{s} \mathscr{B} \mathscr{S}_{[\mathrm{q}]}$ be the 2 -quotient of the additive, $\mathbb{C}_{[\mathrm{q}]}$-linear closure of $\boldsymbol{s} \mathscr{B} \mathscr{S}_{[\mathrm{q}]}^{*}$ defined as follows.

'Relations of $\boldsymbol{s} \mathscr{B} \mathscr{S}_{\text {[q] }}$ '. (We only give the relations for one choice of compatible colors and comment on the others choices, where 'Var.: comp. color.' means that the analogous relation holds for other compatible colorings in the sense of (4-2).) 
First, polynomial multiplication, i.e. polynomial decorations on a facet multiply, and isotopy relations:

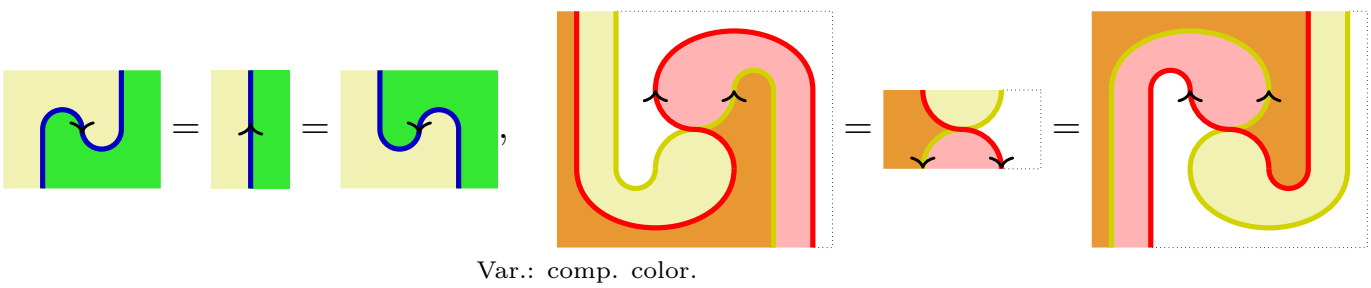

Then various relations involving circles, called circle removals:
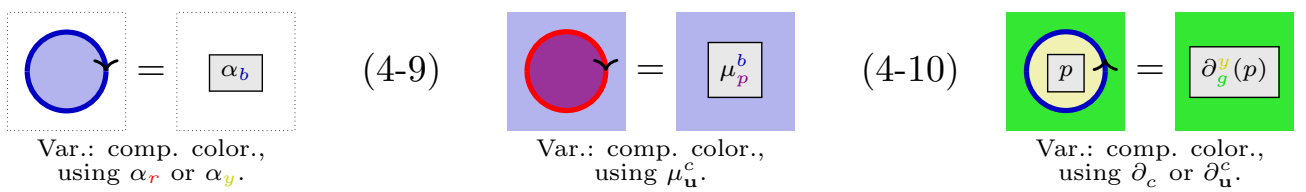

(Note that there is also a variation of (4-10) with a circular $\emptyset$-region in the middle bounded by a primary colored region outside.)

Moreover, we have polynomial sliding and neck cutting relations, i.e.
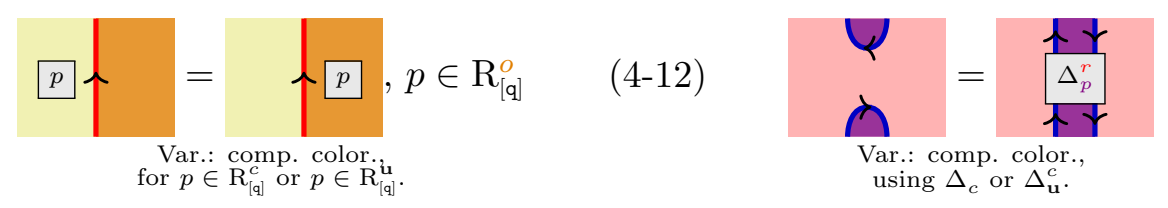

The notation in the neck cutting relations (4-12) indicates that one has put the tensor factors of the various summands of the $\Delta^{-}$in the corresponding regions (i.e. left tensor factors in the leftmost region and right tensor factors in the rightmost region), cf. Example 4.22.

Next, Reidemeister-like relations:
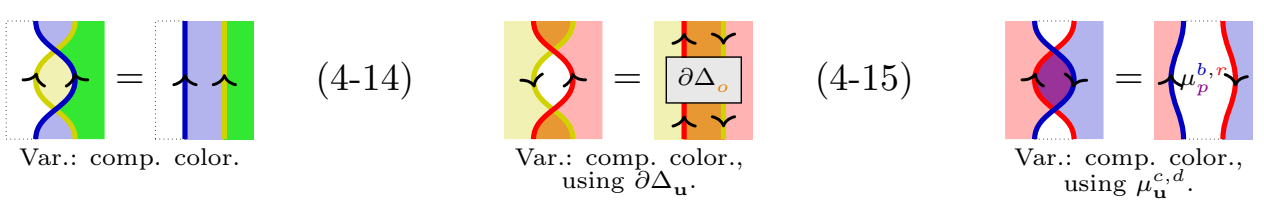

where we use the same conventions for (4-14) as for (4-12).

Finally, the square relations, which we exemplify by the case in which $y \emptyset b$ is at the bottom and $b \emptyset y$ is at the top:

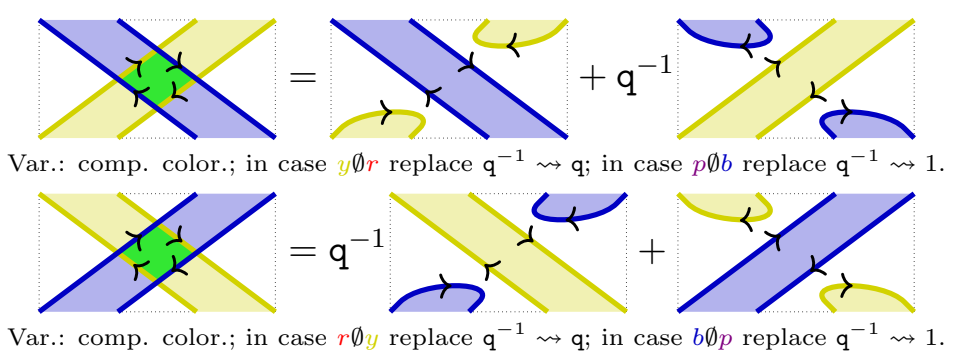

(We stress that (4-16) is not invariant under color change.)

Definition 4.17. The 2-category of regular Bott-Samelson bimodules is defined as

$$
\mathscr{B} \mathscr{S}_{[\mathrm{q}]}=\boldsymbol{s} \mathscr{B} \mathscr{S}_{[\mathrm{q}]}(\emptyset, \emptyset),
$$

i.e. the 2-full 2-subcategory of $\boldsymbol{s} \mathscr{B} \mathscr{S}_{\text {[q] }}$ generated by diagrams whose left- and rightmost color is $\emptyset$. Note that $\mathscr{B} \mathscr{S}_{\text {[q] }}$ has only one object, namely $\emptyset$. 
The 2-category of maximally singular Bott-Samelson bimodules is defined as

$$
\boldsymbol{m} \mathscr{B} \mathscr{S}_{[\mathrm{q}]}=\bigoplus_{\mathbf{u}, \mathbf{v} \in G O P} \boldsymbol{s} \mathscr{B} \mathscr{S}_{[\mathrm{qq}]}(\mathbf{u}, \mathbf{v}),
$$

i.e. the 2-full 2-subcategory of $\boldsymbol{s} \mathscr{B} \mathscr{S}_{\text {[q] }}$ generated by diagrams whose left- and rightmost colors are secondary.

Note that we can always extend scalars to e.g. $\mathbb{C}_{\mathrm{q}}=\mathbb{C}(\mathrm{q})$ and we indicate this by changing the subscript $[\mathrm{q}]$ to $\mathrm{q}$.

Remark 4.18. $\boldsymbol{s} \mathscr{B} \mathscr{S}_{[\mathrm{q}]}$ is an additive, $\mathbb{C}_{[\mathrm{q}]}$-linear, graded 2-category, which is, however, not idempotent closed. This is remedied by considering its Karoubi envelope $\mathscr{K} \operatorname{ar}\left(\boldsymbol{s} \mathscr{B} \mathscr{S}_{[\mathrm{q}]}\right)$, which we take as the definition of the 2-category of Soergel bimodules of affine type $A_{2}$.

Thus, we have:

- The 2-category of singular Bott-Samelson bimodules, whose notation contains an $s$.

- The 2-category of regular Bott-Samelson bimodules, whose notation has no $s$.

- The 2-category of maximally singular Bott-Samelson bimodules, whose notation contains an $\boldsymbol{m}$. As we will see, the degree-zero part of this 2-subcategory, for a fixed choice of shifts of the 1-morphisms, is semisimple.

- The corresponding 2-categories of Soergel bimodules are the Karoubi envelopes of these, by definition.

- Various scalar extensions of these, indicated by subscripts.

We use similar notations throughout, e.g. for scalar extensions of 2-functors.

Remark 4.19. By [Eli17, Theorem A.1], the decategorification of $\mathscr{K} \operatorname{ar}\left(\boldsymbol{s} \mathscr{B} \mathscr{S}_{\mathrm{q}}\right)$, via the split Grothendieck group, is isomorphic to the affine $A_{2}$ Hecke algebroid. This is a multi object version of the affine Hecke algebra $\mathrm{H}_{\mathrm{v}}$ which we met in Section 3B. Moreover, the 2-full 2-subcategory $\mathscr{K} \operatorname{ar}\left(\mathscr{B} \mathscr{S}_{\mathrm{q}}\right)$ decategorifies to $\mathrm{H}_{\mathrm{v}}$.

Examples and further comments.

Example 4.20. In accordance with (4-2), we have the following Frobenius extensions
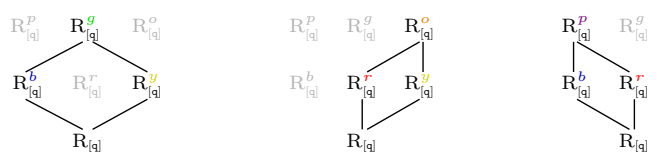

with the corresponding trace maps going upwards. Moreover,

$$
\partial\left(\alpha_{b}\right)=2, \quad \partial_{g}^{b}\left(\mu_{g}^{b}\right)=3, \quad \partial_{g}\left(\mu_{g}\right)=6,
$$

as an easy calculation shows. Similar results hold for other colors.

Note that the numbers in (4-17), which follow from (4-8), (4-9) and (4-10), are precisely the ranks of the corresponding Frobenius extensions.

Example 4.21. When working with $\boldsymbol{s} \mathscr{B} \mathscr{S}_{[\mathrm{q}]}^{*}$, it is important to remember that the polynomial 2 -generators of a given facet are invariant under the action of the parabolic subgroup which corresponds to the color of that region. For example, $\mu_{g}^{y}$ is an element of $\mathrm{R}_{[\mathrm{q}]}^{y}$, and applying $\partial_{g}^{y}$ to it will make it additionally $b$-invariant. In fact,

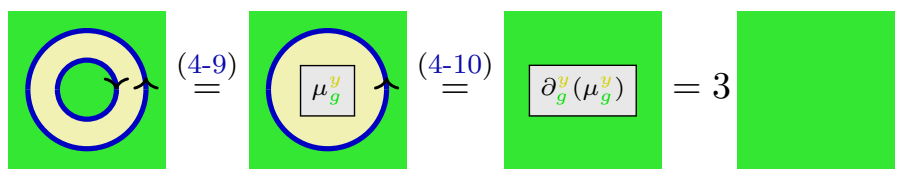


We also get

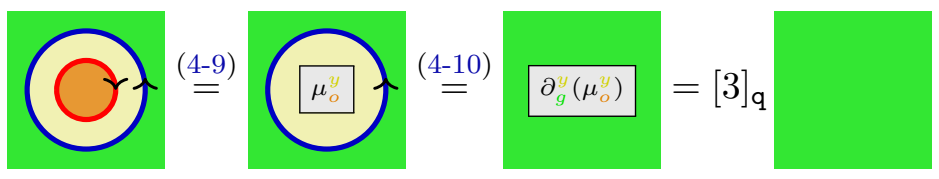

which we will need below.

Example 4.22. We have

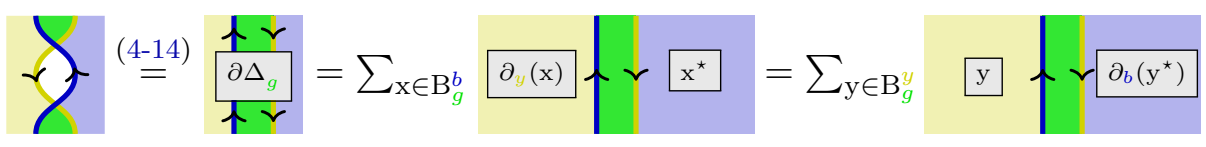

More generally, cf. [Eli17, (3.15d)], the relations in Definition 4.16 imply

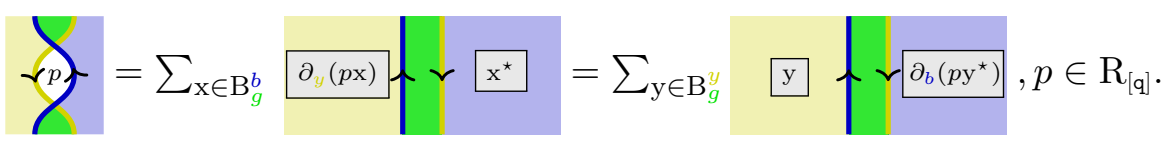

As usual, a similar relations hold for other colors.

\section{B. The trihedral Soergel bimodules of level $\infty$.}

The definition. We first consider a 2-subcategory categorifying $\mathrm{T}_{\infty}$.

Definition 4.23. Let $\mathscr{T}_{\infty,[\mathrm{q}]}$ be the additive closure of the 2 -full 2 -subcategory of $\mathscr{B}_{\text {[q] }}$, whose 1-morphisms are generated by the color strings that have at least one secondary color and have $\emptyset$ as the left- and rightmost color but nowhere else in the string.

Its scalar extension is denoted by $\mathscr{T}_{\infty}=\mathscr{T}_{\infty, \mathrm{q}}$.

Example 4.24. The prototypical 1-morphisms of $\mathscr{T}_{\infty,[\mathrm{q}]}$ are $\emptyset$ and all compatible color variation of

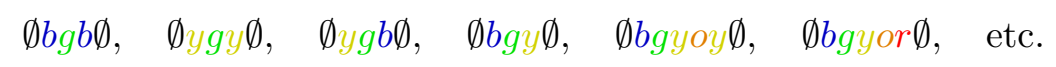

All other 1-morphism in $\mathscr{T}_{\infty,[\mathrm{q}]}$ are direct sums of these, e.g. $\emptyset$ ygyoy $\emptyset \oplus \emptyset$ yoy $\emptyset$.

Some lemmas. We note the following lemma, which follows directly from (4-13).

Lemma 4.25. The following diagrams commute in $\boldsymbol{s} \mathscr{B} \mathscr{S}_{[\mathrm{q}]}$.
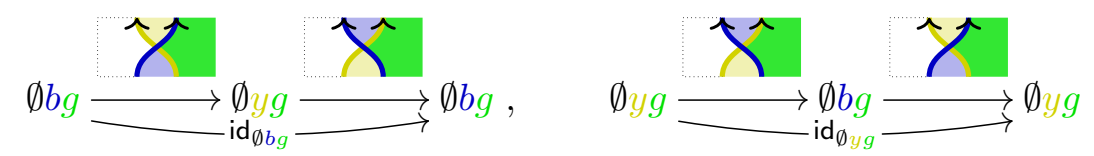

In particular, $\emptyset b g \cong \emptyset y g$. The same holds for color variations with compatible colors.

The following, where we silently use Lemma 4.25, should be compared to Lemma 3.2.

Lemma 4.26. In $\mathscr{K} \operatorname{ar}\left(\mathscr{T}_{\infty}\right)$, the 1-morphism $\emptyset b g b \emptyset \cong \emptyset y g y \emptyset$ is isomorphic to the indecomposable direct summand of $\emptyset b \emptyset y \emptyset b \emptyset \cong \emptyset y \emptyset b \emptyset y \emptyset$ which corresponds to the word $w_{g}=b y b=y b y \in \mathrm{W}_{g}$. The same holds for all compatible color variations.

Recall that $\mathscr{B} \mathscr{S}_{\mathrm{q}}$ decategorifies to $\mathrm{H}_{\mathrm{v}}\left(\tilde{\mathrm{A}}_{2}\right)$ (cf. Remark 4.19), such that the indecomposable 1-morphisms in $\mathscr{B} \mathscr{S}_{\mathrm{q}}$ decategorify to the KL basis elements of the affine type $\mathrm{A}_{2}$ Weyl group W. Since $\mathscr{T}_{\infty}$ is a 2 -full 2-subcategory of $\mathscr{B} \mathscr{S}_{\mathrm{q}}$, its indecomposable 1-morphisms are also indecomposable as 1-morphisms of the latter. Therefore, $\mathscr{K} \operatorname{ar}\left(\mathscr{T}_{\infty}\right)$ decategorifies to a subalgebra of $\mathrm{H}_{\mathrm{v}}\left(\tilde{\mathrm{A}}_{2}\right)$, with a basis consisting of a particular subset of the KL basis elements. 
Proof. By (4-16), we have

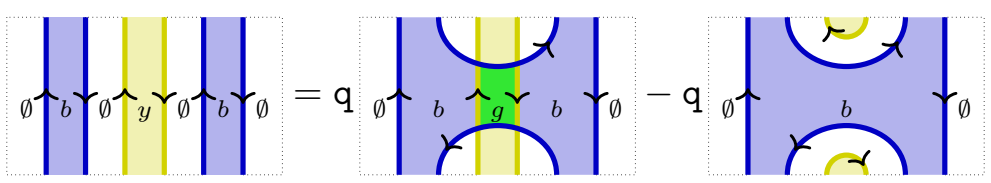

It is not hard to check, using the relations in Definition 4.16, that (4-19) gives a decomposition into orthogonal idempotents.

Note that the first idempotent on the right-hand side shows that $\emptyset b g b \emptyset$ is a direct summand of $\emptyset b \emptyset y \emptyset b \emptyset$, as indicated in (4-19), i.e. $\emptyset b \emptyset y \emptyset b \emptyset \cong \emptyset b g b \emptyset \oplus \emptyset b \emptyset$.

It follows from [Eli17, Theorem A.1], that the idempotents on the right-hand side of (4-19) are primitive and that this decomposition decategorifies to

$$
\theta_{w_{g}} \stackrel{(3-6)}{=} \theta_{b} \theta_{y} \theta_{b}=\theta_{b y b}+\theta_{b} .
$$

This shows the lemma in case of $b, y$ and $g$. The other cases are analogous.

Lemma 4.27. We have $b \emptyset b \cong b\{+1\} \oplus b\{-1\}$ in $\boldsymbol{s} \mathscr{B} \mathscr{S}_{[\mathrm{q}]}$. A similar result holds for all compatible color variations, keeping the color $\emptyset$.

Note that Lemma 4.27 is only true for primary colors, since $\emptyset$ is never compatible to a secondary color.

Proof. This follows from the following diagram.

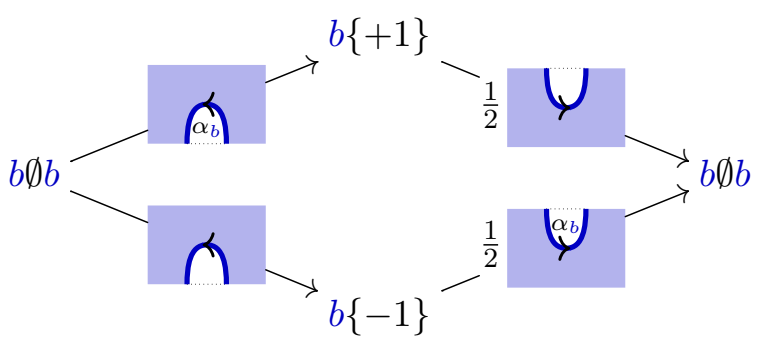

Observing that $\partial_{b}\left(\alpha_{b}\right)=2$ and $\partial_{b}\left(\alpha_{b}^{2}\right)=0$, and using the relations in Definition 4.16, one can check that the left and right column in this diagram define mutually inverses. Similar arguments can be used for all other compatible color variations.

Remark 4.28. Using Lemma 4.27 we simplify our diagrams and do not illustrate $\emptyset$ colored regions in the middle, if not necessary. For example, $\theta_{g} \theta_{g}$ should be thought of as corresponding to $\emptyset b g b g b \emptyset$ instead of $\emptyset b g b \emptyset b g b \emptyset$. However, the appearing grading shift in this simplification is exactly the reason for the scaling by powers of $[2]_{\mathrm{v}}^{-1}$ in Section 3 .

Definition 4.29. Define the following 2-morphisms in $\boldsymbol{s} \mathscr{B} \mathscr{S}_{\text {[q] }}$ (and similar ones for other colors).

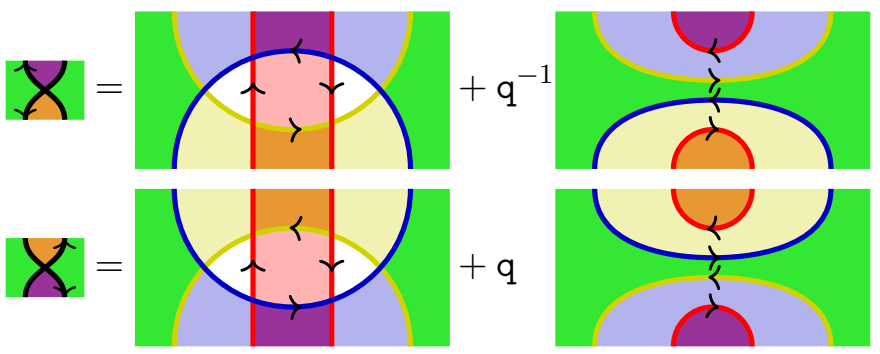

Lemma 4.30. The following diagrams commute in $\boldsymbol{s} \mathscr{B} \mathscr{S}_{[\mathrm{q}]}$.
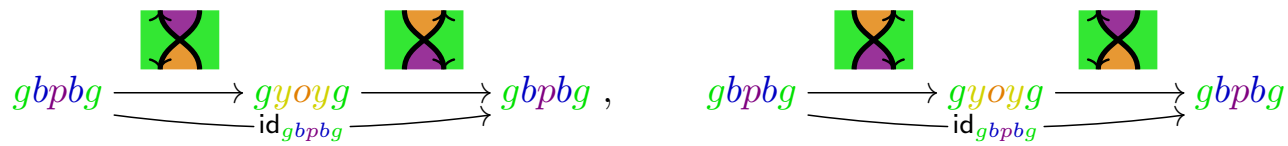
In particular, gyoyg $\cong g b p b g$. A similar result holds for other colors.

Proof. We only prove that the left diagram commutes. To this end, we write $f_{1}$ and $q^{-1} f_{2}$ for the two summands on the right-hand side of the top equality in (4-20), and similarly $g_{1}$ and $\mathrm{qg}_{2}$ for the bottom equality. Using (4-16) we get $\mathrm{g}_{1} \circ_{v} \mathrm{f}_{1}=\mathrm{id}_{g b p b g}+\mathrm{h}$. Moreover, using (4-10) and Example 4.22, we get $\mathrm{g}_{1} \circ_{v} \mathrm{q}^{-1} \mathrm{f}_{2}+\mathrm{qg}_{2} \circ \mathrm{f}_{1}=-[2]_{\mathrm{q}}^{2} h$. Finally, $\mathrm{qg}_{2} \circ{ }_{v} \mathrm{q}^{-1} \mathrm{f}_{2}=[3]_{\mathrm{q}} \mathrm{h}$ follows from Example 4.21, and we are done since $[3]_{\mathrm{q}}=[2]_{\mathrm{q}}^{2}-1$.

Categorifying $\mathrm{T}_{\infty}$

Proposition 4.31. The assignment given by

$$
\theta_{g} \mapsto[\emptyset b g b \emptyset]=[\emptyset y g y \emptyset], \quad \theta_{o} \mapsto[\emptyset \text { ror } \emptyset]=[\emptyset y о y \emptyset], \quad \theta_{p} \mapsto[\emptyset b p b \emptyset]=[\emptyset r p r \emptyset],
$$

defines an isomorphism $\mathrm{T}_{\infty} \cong\left[\mathscr{K} \text { ar }\left(\mathscr{T}_{\infty}\right)\right]_{\oplus}^{\mathbb{C}_{\mathrm{v}}}$ of algebras.

Under this isomorphism, the elements of the basis $\mathrm{C}^{\infty}$ (or of ${ }^{\infty} \mathrm{C}$ ) from Proposition 3.9 correspond to a complete set of indecomposables in $\mathscr{K} \operatorname{ar}\left(\mathscr{T}_{\infty}\right)$ (up to grading shift).

Proof. This follows now directly from Remark 4.19, and Lemmas 3.2, 4.25 and 4.26.

4C. The 2-quotient of level $e$. The quotient $\mathrm{T}_{e}$ of $\mathrm{T}_{\infty}$ from Definition 3.12 is defined by killing certain elements which correspond to the irreducibles $\mathrm{L}_{m, n}$ for $m+n=e+1$ in the representation category of $\mathfrak{s l}_{3}$. We follow the same strategy on the categorified level.

From $\mathfrak{s l}_{3}$ to singular bimodules: the generic case. Recall from Section $2 \mathrm{~A}$ that $\mathcal{Q}_{\mathrm{q}}$ denotes the category of finite-dimensional $\mathrm{U}_{\mathrm{q}}\left(\mathfrak{s l}_{3}\right)$-modules (with $\mathbb{C}_{\mathrm{q}}$ being the ground field). The central character (2-4) allows us to view $\mathcal{Q}_{\mathrm{q}}$ as a 2-category $\mathscr{Q}_{\mathrm{q}}^{G O P}$ :

Definition 4.32. For $\mathbf{u}$, let $\mathcal{Q}_{\mathrm{q}}^{\mathrm{u}}$ denote the full subcategory of $\mathcal{Q}_{\mathrm{q}}$ generated by the irreducibles with central character $\mathbf{u}$.

Note that the subcategories $\mathcal{Q}_{\mathrm{q}}^{\mathbf{u}}$ are not monoidal. However, by Lemma 2.4, tensoring with $\mathrm{X}$ or $\mathrm{Y}$ defines functors between them

$$
\rho(\mathbf{u}) \mathrm{X}_{\mathbf{u}}=\mathrm{X} \otimes_{-}: \mathcal{Q}_{\mathrm{q}}^{\mathbf{u}} \rightarrow \mathcal{Q}_{\mathrm{q}}^{\rho(\mathbf{u})}, \quad \rho^{-1}(\mathbf{u}) \mathrm{Y}_{\mathbf{u}}=\mathrm{Y} \otimes_{-}: \mathcal{Q}_{\mathrm{q}}^{\mathbf{u}} \rightarrow \mathcal{Q}_{\mathrm{q}}^{\rho^{-1}(\mathbf{u})},
$$

which the reader should compare to (3-1). We will (reading right to left) depict them by

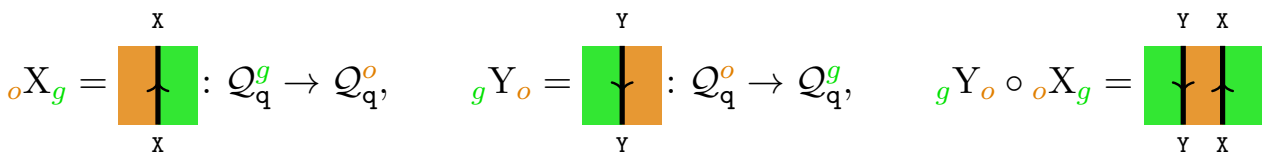

etc. (We omit the $\mathrm{X}$ and the $\mathrm{Y}$ in the pictures from now on.)

Definition 4.33. We define $\mathscr{Q}_{\mathrm{q}}^{G O P}$ to be the additive, $\mathbb{C}_{\mathrm{q}}$-linear closure of the 2-category whose objects are the categories $\mathcal{Q}_{\mathrm{q}}^{\mathrm{u}}$, whose 1-morphisms are composites of the functors in (4-22), and whose 2-morphisms are natural transformations.

A natural transformation between composites of the functors from (4-22) is the same as an $\mathrm{U}_{\mathrm{q}}\left(\mathfrak{s l}_{3}\right)$-equivariant map, see e.g. [EGNO15, Proposition 2.5.4]. Therefore, we define

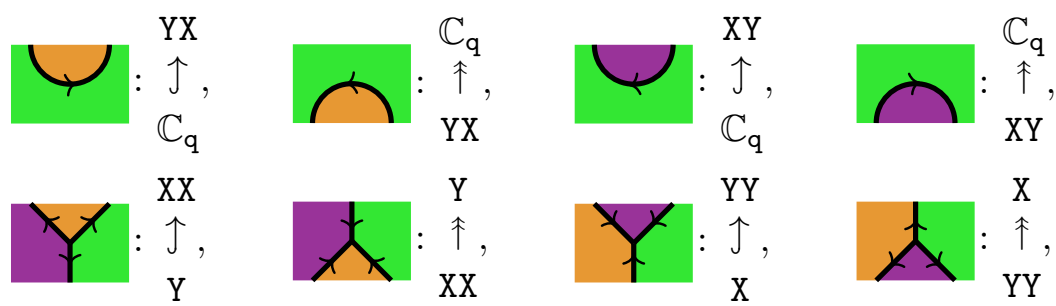


to be the corresponding inclusions respectively projections, which are well-defined up to scalars. We do this in all color variations.

In this way we can view $\mathscr{Q}_{\mathrm{q}}^{G O P}$ as being generated by the diagrams as in (4-23).

Fixing scalars appropriately (which we will do below), it is not hard to see that we get
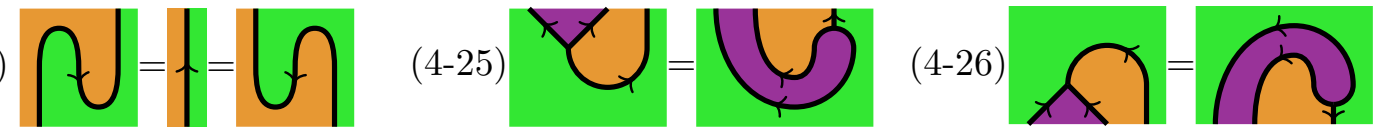

$(4-27)$
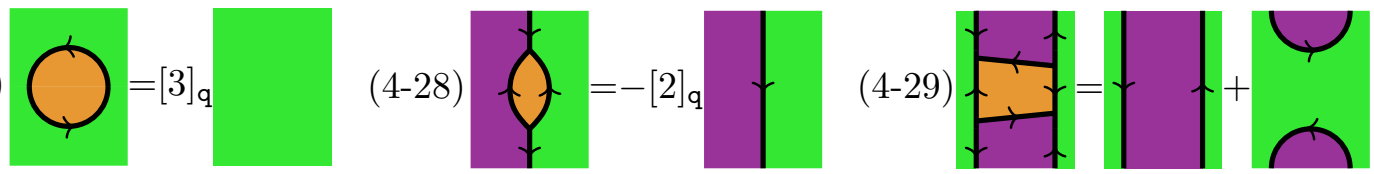

together with those obtained by varying the orientation and the colors, and the vertical mirrors of (4-25) and (4-26).

The following result is a consequence of [Kup96, Theorem 6.1].

Lemma 4.34. The $\mathrm{U}_{\mathrm{q}}\left(\mathfrak{s l}_{3}\right)$-equivariant maps/diagrams from (4-23) together with the relations (4-24) to (4-29) give a generator-relation 2-presentation of $\mathscr{Q}_{\mathrm{q}}^{G O P}$.

Following [Eli17, Section 3] we define a Satake 2-functor.

Definition 4.35. For $\mathbf{u}$ let $\mathrm{S}_{\mathrm{q}}: \mathscr{Q}_{\mathrm{q}}^{G O P} \rightarrow \boldsymbol{m} \mathscr{B} \mathscr{S}_{\mathrm{q}}$ be the 2 -functor defined as follows. On objects by $\mathrm{S}_{\mathrm{q}}\left(\mathcal{Q}_{\mathbf{q}}^{\mathbf{u}}\right)=\mathbf{u}$, on 1-morphisms by $\mathrm{S}_{\mathrm{q}}\left(\rho(\mathbf{u}) \mathrm{X}_{\mathbf{u}}\right)=\rho(\mathbf{u}) c \mathbf{u}$ and $\mathrm{S}_{\mathrm{q}}\left(\rho^{-1}(\mathbf{u}) \mathrm{Y}_{\mathbf{u}}\right)=$ $\rho^{-1}(\mathbf{u}) c \mathbf{u}$, and on 2-morphisms by
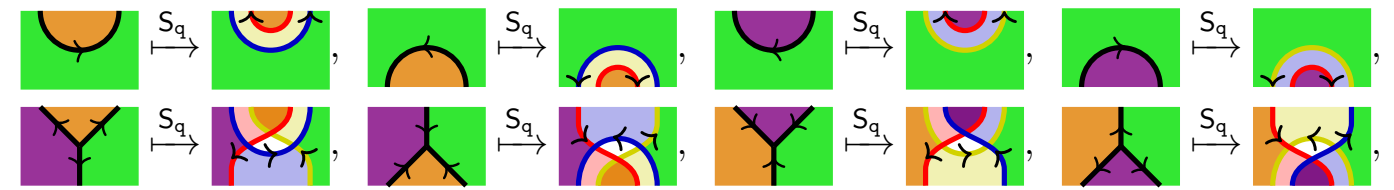

together with similar assignments for the other generators.

The following lemma recalls [Eli17, Claim 3.19]. We sketch its proof for the convenience of the reader and refer to [Eli17, Proof of Claim 3.19] for more details.

Lemma 4.36. The 2-functor $S_{q}$ is well-defined.

Proof. We only need to show that (4-24)-(4-29) hold in the image of $\mathrm{S}_{\mathrm{q}}$. The isotopies (4-24)-(4-26) are clearly preserved. For (4-27) we have already verified this in Example 4.21, while (4-28) follows from (4-15), (4-13) and (4-10) together with $\partial_{b}\left(\mu_{o}^{r, y}\right)=-[2]_{\mathrm{q}}$. The relation (4-29) is a bit more involved (but not hard), and can be proved by using (4-16) on the $\mathrm{S}_{\mathrm{q}}$-image of the square.

We say that a 2-functor from an ungraded 2-category (whose 2-morphisms are all of degree zero, by convention) to a graded 2-category is a degree-zero 2-equivalence, if it is a bijection on objects, essentially surjective on 1-morphisms, faithful on 2-morphisms, and full onto degree-zero 2-morphisms. Using this notion, the quantum Satake correspondence can be formulated as in [Eli17, Theorem 3.21]:

Theorem 4.37. The 2-functor $\mathrm{S}_{\mathrm{q}}$ is a degree-zero 2-equivalence.

Remark 4.38. Elias actually proves Theorem 4.37 in much more generality. For us the important case is over the ring $\mathbb{C}_{[\mathrm{q}]}=\mathbb{C}\left[\mathbf{q}, \mathrm{q}^{-1}\right]$, which then implies that Theorem 4.37 holds over any ground ring we are going to use. 
Let $\mathbf{p}_{g}^{m, n}=\mathbf{p}_{g}^{m, n}\left(\mathrm{X}^{m} \mathrm{Y}^{n}\right)$ denote the (unique) projection of $\mathrm{X}^{m} \mathrm{Y}^{n}$ onto the irreducible direct summand $\mathrm{L}_{m, n}$, regarded as a 2-morphism in $\mathscr{Q}_{\mathrm{q}}^{G O P}$ with rightmost color $g$. We call $\mathbf{p}_{g}^{m, n}$ the (right-green) $\mathfrak{s l}_{3}$-clasp. Similarly, we define $\mathbf{p}_{o}^{m, n}, \mathbf{p}_{p}^{m, n}$ and ${ }^{m, n} \mathrm{p},{ }_{o}^{m, n} \mathrm{p},{ }_{p}^{m, n} \mathrm{p}$. Note that there is actually a different clasp for each product of $m$ factors $\mathrm{X}$ and $n$ factors $\mathrm{Y}$, but these clasps are all closely related, as we will see in Lemma 4.42. For now, it suffices to consider only the one for $\mathrm{X}^{m} \mathrm{Y}^{n}$.

Remark 4.39. The $\mathfrak{s l}_{3}$-clasps have a diagrammatic incarnation, obtained by coloring the diagrammatic clasps from [Kim07, Theorem 3.3] (which gives the $\mathfrak{s l}_{3}$-clasps in terms of a recursion), or (using slightly different conventions) from [Eli15, (1.8) and Section 3.2].

The colored $\mathfrak{s l}_{3}$-clasps are 2-morphisms in $\boldsymbol{m} \mathscr{B} \mathscr{S}_{\mathrm{q}}$, but do not belong to $\mathscr{T}_{\infty}$, since their left- and rightmost colors are always secondary colors. Thus, we need to 'biinduce them up to $\emptyset^{\prime}$ in order to have their appropriate analogs in $\mathscr{T}_{\infty}$ :

Definition 4.40. The colored (right-u) clasps $c_{\mathbf{u}}^{m, n}$ are defined by

$$
c_{\mathbf{u}}^{m, n}=\mathrm{id}_{\emptyset d \mathbf{v}} \circ_{h} \mathrm{p}_{\mathbf{u}}^{m, n} \circ_{h} \mathrm{id}_{\mathbf{u c} \emptyset}
$$

with $o_{h}$ being the horizontal composition in $\boldsymbol{s} \mathscr{B} \mathscr{S}_{\mathbf{q}}$. Here $\mathrm{p}_{\mathbf{u}}^{m, n}$ has leftmost color $\mathbf{v}$, and $c, d$ are compatible colors where we prefer $b$ over $r$ over $y$. We define ${ }_{\mathbf{u}}^{m, n} \mathrm{c}$ similarly.

The colored clasps are idempotent 2-morphisms in $\mathscr{T}_{\infty}$, which depend on the same choices as the colored $\mathfrak{s l}_{3}$-clasps and, additionally, on the choice of $c, \mathbf{u}$. Again, this dependence is not essential, as we will show in Lemma 4.42, so we abuse notation.

Example 4.41. Using [Kim07, Theorem 3.3], one can write down the colored $\mathfrak{s l}_{3}$-clasps explicitly, e.g. in the case $e+1=2$ :

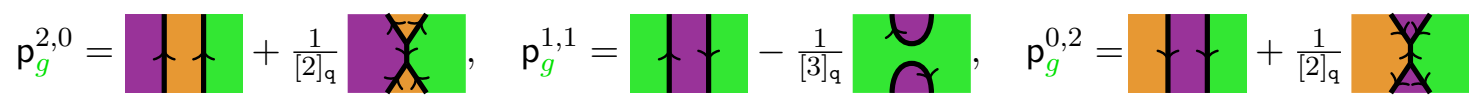

Using $S_{q}$ and biinduction, we get for example:

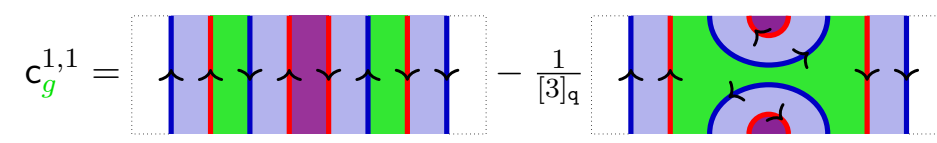

(The outer $b$-regions come from our choice in Definition 4.40.)

The next lemma shows that the colored clasps are independent of the choices that we made in their definition:

Lemma 4.42. Let $c_{\mathbf{u}}^{m, n}$ be a colored clasps and let $\left(c_{\mathbf{u}}^{m, n}\right)^{\prime}$ be defined similarly, but with some difference in the involved choices. Then there exists an invertible 2 -morphisms $f$ in $\mathscr{T}_{\infty}$ such that $c_{\mathbf{u}}^{m, n} \circ_{v} f=f \circ_{v}\left(c_{\mathbf{u}}^{m, n}\right)^{\prime}$.

Proof. If the ordering of the factors $\mathrm{X}$ and $\mathrm{Y}$ for $c_{\mathbf{u}}^{m, n}$ and $\left(c_{\mathbf{u}}^{m, n}\right)^{\prime}$ differs by precisely one pair, then Lemma 4.30 shows the claim. If $c_{\mathbf{u}}^{m, n}$ and $\left(c_{\mathbf{u}}^{m, n}\right)^{\prime}$ differ by precisely one choice of compatible color for 'biinduction', then Lemma 4.25 shows the claim. Then the general statement then follows by induction.

Corollary 4.43. If a two sided 2-ideal in $\mathscr{T}_{\infty}$ contains a colored clasp $c_{\mathbf{u}}^{m, n}$, then it contains all colored clasps $\left(c_{\mathbf{u}}^{m, n}\right)^{\prime}$ which differ from $c_{\mathbf{u}}^{m, n}$ by some of the choices involved in their definition. 
From $\mathfrak{s l}_{3}$ to singular bimodules: the root of unity case. From now on we work over $\mathbb{C}$ by specializing q to $\eta$ which, as usual, is a $2(e+3)^{\text {th }}$ primitive, complex root of unity. Formally this is done by repeating the above for the $\mathbb{C}_{[\mathrm{q}]}$ linear 2-categories which are scalar extended to $\mathbb{C}_{[e]}=\mathbb{C}\left[\mathbf{q}, \mathrm{q}^{-1},[2]_{\mathrm{q}}^{-1}, \ldots,[e+1]_{\mathrm{q}}^{-1}\right]$. We denote these using $[e]$ as a subscript, and the specialization at $\mathrm{q}=\eta$ we denote by $\otimes_{\mathbb{C}_{[e]}} \mathbb{C}$. We also exclude the case $e=0$, which is a bit special and can easily be dealt with later on.

First of all, all previous definitions and results in the generic case are still valid in this case, except Lemma 4.34 (which we do not need in the following) and the definition of the (various) clasps for $m+n>e+1$. In particular, Lemma 4.36 and Theorem 4.37 still hold in this case.

Lemma 4.44. The colored clasps are well-defined in $\mathscr{T}_{[e]}$ (seen as a 2 -subcategory of $\mathscr{B} \mathscr{S}_{[e]}$ ) for $0 \leq m+n \leq e+1$, and uniquely determined up to conjugation by an invertible 2-morphism.

Proof. Decomposing $\mathrm{X}^{m} \mathrm{Y}^{n}$ generically, i.e. for $\mathrm{U}_{\mathrm{q}}\left(\mathfrak{s l}_{3}\right)$, works similarly as in the root of unity case as long as $m+n \leq e+1$, see e.g. [AP95, Section 3]. Thus, one can use the specializations of the projectors from the generic case in the root of unity case. (Alternatively, using Remark 4.39, one checks that the coefficients of the colored $\mathfrak{s l}_{3}$-clasps specialize properly.) Finally, note that Lemmas 4.36 and 4.42 also hold for q being specialized to $\eta$.

Having all the above established, we can define the 2-category of trihedral Soergel bimodules of level $e$ :

Definition 4.45. Let $\mathscr{I}_{e}$ be the two-sided 2-ideal, called vanishing 2 -ideal of level $e$, in $\mathscr{T}_{[e]} \otimes_{\mathbb{C}_{[e]}} \mathbb{C}$ generated by

$$
\left\{\mathbf{c}_{\mathbf{u}}^{m, n} \mid m+n=e+1, \mathbf{u} \in G O P\right\}=\left\{{ }^{m, n} \mathbf{u} \mathbf{c} \mid m+n=e+1, \mathbf{u} \in G O P\right\},
$$

where we write e.g. $c_{\mathbf{u}}^{m, n}=c_{\mathbf{u}}^{m, n} \otimes_{\mathbb{C}_{[e]}} 1$ for simplicity. We define

$$
\mathscr{T}_{e}=\left(\mathscr{T}_{[e]} \otimes_{\mathbb{C}_{[e]}} \mathbb{C}\right) / \mathscr{I}_{e},
$$

which we call the 2-category of trihedral Soergel bimodules of level $e$.

Remark 4.46. Note that we specialize before taking the quotient, as Andersen-Paradowski do in order to define $\mathcal{Q}_{e}$ in [AP95], where they take the quotient of the already specialized category $\mathcal{Q}_{\eta}$ by the ideal of so-called negligible modules. (This is explicitly described in e.g. [BK01, Section 3.3].) Similarly, we always specialize first throughout.

Let $\mathscr{H}_{e}$ be the two-sided 2-ideal in $\mathscr{Q}_{[e]}^{G O P} \otimes_{\mathbb{C}_{[e]}} \mathbb{C}$ generated by

$$
\left\{\mathrm{p}_{\mathbf{u}}^{m, n} \mid m+n=e+1, \mathbf{u} \in G O P\right\}=\{\stackrel{m, n}{\mathbf{u}} \mathbf{p} \mid m+n=e+1, \mathbf{u} \in G O P\} .
$$

The maximally singular version of $\mathscr{T}_{e}$ is

$$
\boldsymbol{m} \mathscr{T}_{e}=\left(\boldsymbol{m} \mathscr{T}_{[e]} \otimes_{\mathbb{C}_{[e]}} \mathbb{C}\right) / \mathscr{H}_{e}
$$

Here we again specialize $\mathrm{q}$ to $\eta$ and use the same conventions as before. We state a non-trivial consequence of the quantum Satake correspondence from Theorem 4.37 and Remark 4.38.

Lemma 4.47. $\mathrm{S}_{[e]}$ gives rise to a degree-zero 2-equivalence $\mathrm{S}_{e}: \mathscr{Q}_{e}^{G O P} \rightarrow \boldsymbol{m} \mathscr{T}_{e}$.

Proof. Because $\mathrm{S}_{[e]}$ is a degree-zero 2-equivalence before quotienting by any clasps, by Remark 4.38, it sends indecomposable 1-morphisms in $\mathscr{Q}_{[e]}^{G O P}$ to indecomposable 1-morphisms in $\boldsymbol{m} \mathscr{T}_{[e]}$, and the cell structures of $\mathscr{Q}_{[e]}^{G O P}$ and $\boldsymbol{m} \mathscr{T}_{[e]}$ are isomorphic under $\mathrm{S}_{[e]}$.

Clearly, $\mathrm{S}_{[e]}$ specializes to $\mathrm{S}_{\eta}: \boldsymbol{m} \mathscr{T}_{[e]} \otimes_{\mathbb{C}_{[e]}} \mathbb{C} \rightarrow \mathscr{Q}_{[e]}^{G O P} \otimes_{\mathbb{C}_{[e]}} \mathbb{C}$, which descends to the 2-functor $\mathrm{S}_{e}: \mathscr{Q}_{e}^{G O P} \rightarrow \boldsymbol{m} \mathscr{T}_{e}$. Both $\mathrm{S}_{\eta}$ and $\mathrm{S}_{e}$ are essentially surjective on 1-morphisms and full onto degree-zero 2-morphisms. What is not immediately clear, is that $\mathrm{S}_{e}: \mathscr{Q}_{e}^{G O P} \rightarrow \boldsymbol{m} \mathscr{T}_{e}$ is also 
faithful on 2-morphisms: since $\mathscr{T}_{[e]}$ has 2-morphisms of negative degree, the degree-zero part of $\mathscr{I}_{e}$ could a priori be bigger than $S_{\eta}\left(\mathscr{H}_{e}\right)$. To show that that is not the case, we use the following roundabout argument.

From [AP95] (cf. Remark 4.46) we know that we have an equivalence of 2-categories

$$
\left(\mathscr{Q}_{[e]}^{G O P} \otimes_{\mathbb{C}_{[e]}} \mathbb{C}\right) / \mathscr{H}_{e} \cong \mathscr{Q}_{e}^{G O P},
$$

Hence, the indecomposable 1-morphisms $\mathrm{F}$ in $\mathscr{Q}_{[e]}^{G O P} \otimes_{\mathbb{C}_{[e]}} \mathbb{C}$ for which id $\in \mathscr{H}_{e}$ are strictly greater than the ones for which id $\notin \mathscr{H}_{e}$, in the two-sided order. By the observations in the first paragraph, the same must hold for the indecomposable 1-morphisms in $\mathscr{T}_{[e]} \otimes \mathbb{C}_{[e]} \mathbb{C}$ with respect to the 2-ideal $\mathscr{I}_{e}$, which is generated by $\mathrm{S}_{\eta}\left(\mathscr{H}_{e}\right)$. This shows that $\mathrm{S}_{e}: \mathscr{Q}_{e}^{G O P} \rightarrow \boldsymbol{m} \mathscr{T}_{e}$ is faithful on 2-morphisms, since $\mathscr{Q}_{e}^{G O P}$ is semisimple.

Proposition 4.48. The isomorphism from Proposition 4.31 gives an isomorphism $\mathrm{T}_{e} \cong$ $\left[\mathscr{K} \operatorname{ar}\left(\mathscr{T}_{e}\right)\right]_{\oplus}^{\mathbb{C}_{\mathrm{v}}}$ of algebras.

Proof. This follows from the discussion above: By Corollary 4.43 and Lemma 4.44 the vanishing 2-ideal of level $e$ contains all colored clasps of level $e+1$. By by Proposition 4.31 these decategorify to the $c_{\mathbf{u}}^{m, n}$ in the definition of $\mathrm{T}_{e}$, while Lemma 4.47 ensures that the Grothendieck classes of the remaining $c_{\mathbf{u}}^{m, n}$ form a basis of $\left[\mathscr{K} \operatorname{ar}\left(\mathscr{T}_{e}\right)\right]_{\oplus}^{\mathbb{C}_{\mathbf{v}}}$.

4D. Generalizing dihedral Soergel bimodules. As before, we list certain analogies to the dihedral case.

The dihedral story 4.49. The Hecke algebra $H_{v}\left(\tilde{A}_{1}\right)$ of 'The dihedral story' 3.22 is categorified by Soergel bimodules of affine type $A_{1}$. Here the Hecke algebra $H_{v}\left(\tilde{A}_{2}\right)$ is categorified by Soergel bimodules of affine type $A_{2}$. The difference is that now biinduction of the maximally singular bimodules only gives a proper 2 -subcategory.

The dihedral story 4.50. The Satake 2-functor from (4-30) exists in the dihedral case as well, with a bicolored version of quantum $\mathfrak{s l}_{2}$-modules as the source 2-category. This 2-category has a diagrammatic incarnation in terms of a 2-colored Temperley-Lieb calculus [Eli16, Section 4.3]. The Soergel bimodules of finite Coxeter type $I_{2}(e+2)$ can then be defined by annihilating the ideal generated by the colored Jones-Wenzl projectors (i.e. colored $\mathfrak{s l}_{2}$ clasps) of level $e+1$ in this 2-colored Temperley-Lieb calculus. Moreover, while the colored $\mathfrak{s l}_{3}$-clasps satisfy the recursion in Lemma 2.7, their $\mathfrak{s l}_{2}$ counterparts satisfy the Chebyshev recursion from 'The dihedral story' 3.24. Finally, the analogs of Propositions 4.31 and 4.48 hold as well.

\section{Missing proofs from Section 3.}

Proof of Lemma 3.7. Recall that the 2-category $\mathscr{B} \mathscr{S}_{\mathrm{q}}$ categorifies $\mathrm{H}_{\mathrm{v}}$, i.e.

$$
\mathrm{H}_{\mathrm{v}} \cong\left[\mathscr{K} \operatorname{ar}\left(\mathscr{B} \mathscr{S}_{\mathrm{q}}\right)\right]_{\oplus}^{\mathbb{C}_{\mathrm{v}}}
$$

such that the KL elements are sent to the Grothendieck classes of the indecomposable 1morphisms (with a fixed choice of grading), c.f. [Eli17], [EW16] or Remark 4.19. Furthermore, by Lemma 3.2, the algebra $\mathrm{T}_{\infty}$ can be embedded into $\mathrm{H}_{\mathrm{v}}$ by sending the colored KL elements in $\mathrm{T}_{\infty}$ to KL elements in $\mathrm{H}_{\mathrm{v}}$. Thus, we can identify elements of $\mathrm{T}_{\infty}$ with Grothendieck classes in $\left[\mathscr{K} \operatorname{ar}\left(\mathscr{B} \mathscr{S}_{\mathrm{q}}\right)\right]_{\oplus}^{\mathbb{C}_{\mathrm{v}}}$ :

The element $\mathrm{h}_{\mathbf{u}}^{k, l}=\theta_{\mathbf{u}_{k+l}} \cdots \theta_{\mathbf{u}_{1}} \theta_{\mathbf{u}_{0}} \in \mathrm{H}_{\mathrm{v}}$, with $\mathbf{u}_{0}=\mathbf{u}$, corresponds to

$$
\left[\emptyset c_{k+l} \mathbf{u}_{k+l} c_{k+l} \emptyset \cdots \emptyset c_{1} \mathbf{u}_{1} c_{1} \emptyset c_{0} \mathbf{u}_{0} c_{0} \emptyset\right] \in\left[\mathscr{K} \operatorname{ar}\left(\mathscr{B} \mathscr{S}_{\mathrm{q}}\right)\right]_{\oplus}^{\mathbb{C}_{\mathrm{v}}},
$$

where we can chose any compatible primary colors by Lemma 4.25. In fact, with Lemma 4.25 in mind, we will denote all compatible primary colors simply by $c$ from now on. 
Using Lemma 4.27, we see that the element in (4-32) is equal to

$$
[2]_{\mathrm{v}}^{k+l}\left[\emptyset c \mathbf{u}_{k+l} c \cdots c \mathbf{u}_{1} c \mathbf{u}_{0} c \emptyset\right] \in\left[\mathscr{K} \operatorname{ar}\left(\mathscr{B} \mathscr{S}_{\mathrm{q}}\right)\right]_{\oplus}^{\mathbb{C}_{\mathrm{v}}} .
$$

Next, we use $\mathrm{S}_{\mathrm{q}}$ from (4-30). By definition, $\left[\mathrm{S}_{\mathrm{q}}\right] \operatorname{maps}\left[\mathrm{X}^{k} \mathrm{Y}^{l}\right] \in\left[\mathscr{Q}_{\mathrm{q}}^{G O P}\right]_{\oplus}^{\mathbb{C}_{\mathrm{v}}}$ to

$$
\left[\emptyset c \mathbf{u}_{k+l} c \cdots c \mathbf{u}_{1} c \mathbf{u}_{0} c \emptyset\right] \in\left[\mathscr{K} \operatorname{ar}\left(\mathscr{B} \mathscr{S}_{\mathrm{q}}\right)\right]_{\oplus}^{\mathbb{C}_{\mathrm{v}}},
$$

and to similar expressions with different rightmost color. By Remark 2.2, this implies that $\left[\mathrm{S}_{\mathrm{q}}\right]$ maps $\left[\mathrm{L}_{m, n}\right]$ to

$$
\sum_{k, l}[2]_{\mathrm{v}}^{-k-l} d_{m, n}^{k, l}\left[\emptyset c \mathbf{u}_{k+l} c \emptyset \cdots \emptyset c \mathbf{u}_{1} c \emptyset c \mathbf{u}_{0} c \emptyset\right]
$$

(and again to similar expressions with different rightmost color).

By Theorem 4.37, $\mathrm{S}_{\mathrm{q}}$ is a degree-zero 2-equivalence. In particular, it sends the simple 1-morphisms to indecomposable 1-morphisms. This implies that the element in (4-33) is the Grothendieck class of an indecomposable 1-morphism of $\boldsymbol{s} \mathscr{B} \mathscr{S}_{\mathrm{q}}$. Biinduction preserves indecomposibility, so our element $c_{\mathbf{u}}^{m, n}$ corresponds to the Grothendieck class of an indecomposable 1-morphism in $\left[\mathscr{B S}_{\mathrm{q}}\right]_{\oplus}^{\mathbb{C}_{\mathrm{v}}}$. By the categorification theorem from (4-31), we see that $\mathrm{c}_{\mathbf{u}}^{m, n}$ corresponds to a $\mathrm{KL}$ basis element in $\mathrm{H}_{\mathrm{v}}$, and $\mathrm{h}_{\mathbf{u}}^{k, l}$ to the Grothendieck class of a Bott-Samelson bimodule.

From the above, we obtain the first equation in (3-14), since

$$
[\emptyset c \mathbf{u} c \emptyset c \mathbf{u} c \emptyset]=[2]_{\mathrm{v}}[\emptyset c \mathbf{u} c \mathbf{u} c \emptyset]=[3]_{\mathrm{v}} ![\emptyset c \mathbf{u} c \emptyset]
$$

by Lemma 4.27 and Example 4.21. Using $\left[\mathrm{S}_{\mathrm{q}}\right]$ and (2-6), we deduce the second equation in (3-14). Similarly, one can prove the third equation in (3-14) using (2-7).

Proof of Proposition 3.9. Let $\mathbf{u}$ be fixed for now. Recalling the notation from Section 2, by Lemma 3.3 and its proof given above, there is a $\mathbb{C}_{\mathrm{v}}$-linear isomorphism between the scalar extension $\left[\mathscr{Q}_{\mathrm{q}}^{G O P}\right]_{\oplus}^{\mathbb{C}_{\mathrm{v}}}$ and $\mathbb{C}_{\mathbf{v}}\left\{\mathrm{h}_{\mathbf{u}}^{k, l} \mid(k, l) \in X^{+}\right\}$, defined by

$$
\left[\mathrm{X}^{k} \mathrm{Y}^{l}\right] \mapsto[2]_{\mathrm{v}}^{-k-l} \mathrm{~h}_{\mathbf{u}}^{k, l}
$$

This shows that the $\mathrm{h}_{\mathbf{u}}^{k, l}$ are all linearly independent, and they are also linearly independent of 1 , of course.

Since $\mathbf{u}$ was arbitrary and there are no relations in $\mathrm{T}_{\infty}$ which allow us to change the rightmost color in a word, it follows that

is a basis of $\mathrm{T}_{\infty}$.

$$
\{1\} \cup\left\{\mathrm{h}_{\mathbf{u}}^{k, l} \mid(k, l) \in X^{+}, \mathbf{u} \in G O P\right\}
$$

Because $d_{m, n}^{m, n}=1$, and $d_{m, n}^{k, l}=0$ if $k+l>m+n$, the above immediately implies that

$$
\left\{\mathrm{c}_{\mathbf{u}}^{m, n} \mid(m, n) \in X^{+}, \mathbf{u} \in G O P\right\}
$$

is also a basis, since the transformation between the two sets of elements defined by (3-12) is triangular with diagonal factors $[2]_{\mathrm{v}}^{-m-n} \neq 0$.

\section{TRIHEDRAL 2-REPRESENTATION THEORY}

Keeping all notations from the previous sections, we are now going to explain the 2-representation theory of the trihedral Soergel bimodules. Again, we have collected the analogies to the dihedral case at the end in Section 5D.

\section{Background.}

Let us briefly recall some terminology and results from 2-representation theory as in e.g. [MM16a] or [MM16b], where we also need the graded setup as in [MT16, Section 3]. 
$\mathbb{N}_{[\mathrm{v}]}^{0}$-representation theory. We start with the decategorified picture. Recall that $\mathrm{v}$ denotes a generic parameter, $\mathbb{N}_{[\mathrm{v}]}^{0}=\mathbb{N}^{0}\left[\mathrm{v}, \mathrm{v}^{-1}\right], \mathbb{Z}_{[\mathrm{v}]}=\mathbb{Z}\left[\mathrm{v}, \mathrm{v}^{-1}\right]$ and $\mathbb{C}_{\mathrm{v}}=\mathbb{C}(\mathrm{v})$.

Following various authors, see e.g. [EK95, Section 1], [EGNO15, Chapter 3] or [KM16] and the references therein, we define:

Definition 5.1. A pair $\left(\mathrm{P}, \mathrm{B}^{\mathrm{P}}\right)$ of an associative, unital $\left(\mathbb{C}_{\mathrm{v}^{-}}\right)$algebra $\mathrm{P}$ and a finite basis $\mathrm{B}^{\mathrm{P}}$ with $1 \in \mathrm{B}^{\mathrm{P}}$ is called a $\mathbb{N}_{[\mathrm{v}]}^{0}$-algebra if

$$
\mathrm{xy} \in \mathbb{N}_{[\mathrm{v}]}^{0} \mathrm{~B}^{\mathrm{P}}
$$

holds for all $\mathrm{x}, \mathrm{y} \in \mathrm{B}^{\mathrm{P}}$.

Definition 5.2. Let $\left(\mathrm{M}, \mathrm{B}^{\mathrm{M}}\right)$ be a pair of a (left) $\left(\mathrm{P}, \mathrm{B}^{\mathrm{P}}\right)$-representation $\mathrm{M}$ and a choice of a finite basis $\mathrm{B}^{\mathrm{M}}$ for it. We call $\left(\mathrm{M}, \mathrm{B}^{\mathrm{M}}\right)$ a $\mathbb{N}_{[\mathrm{v}]}^{0}-$ representation if

$$
\mathrm{M}(\mathrm{z}) \mathrm{m} \in \mathbb{N}_{[\mathrm{v}]}^{0} \mathrm{~B}^{\mathrm{M}}
$$

holds for all $\mathrm{z} \in \mathrm{B}^{\mathrm{P}}, \mathrm{m} \in \mathrm{B}^{\mathrm{M}}$.

Example 5.3. These $\mathbb{N}_{[\mathrm{v}]}^{0}$-algebras and $\mathbb{N}_{[\mathrm{v}]}^{0}$-representations arise naturally as the decategorification of 2-categories and 2-representations, which will be recalled in the next section.

Abusing notation, we sometimes write $\mathrm{P}$ instead of $\left(\mathrm{P}, \mathrm{B}^{\mathrm{P}}\right)$ and $\mathrm{M}$ instead of $\left(\mathrm{M}, \mathrm{B}^{\mathrm{M}}\right)$ when the choice of bases is clear from the context.

Definition 5.4. Two $\mathbb{N}_{[\mathrm{v}]}^{0}$-representations $\mathrm{M}, \mathrm{M}^{\prime}$ are $\mathbb{N}_{[\mathrm{v}]}^{0}$-equivalent, denoted by $\mathrm{M} \cong \mathrm{M}_{+}^{\prime}$, if there exist a bijection $\mathrm{B}^{\mathrm{M}} \rightarrow \mathrm{B}^{\mathrm{M}^{\prime}}$ such that the induced linear map $\mathrm{M} \rightarrow \mathrm{M}^{\prime}$ is an isomorphism of P-representations.

Example 5.5. $\mathrm{M} \cong{ }_{+} \mathrm{M}^{\prime}$ implies $\mathrm{M} \cong \mathrm{M}^{\prime}$ (meaning that the are isomorphic as P-representations over $\mathbb{C}_{\mathrm{v}}$ ), but not vice versa:

First of all, $\mathrm{M}$ might be isomorphic over $\mathbb{C}_{\mathrm{v}}$ to a P-representation $\mathrm{M}^{\prime}$ that is not a $\mathbb{N}_{[\mathrm{vl}}^{0}$-representation. For example, consider the $\left(\mathbb{C}_{\mathrm{v}^{-}}\right)$group algebra of any finite group with its basis given by the group elements. Its regular representation is a $\mathbb{N}_{[\mathrm{v}]}^{0}$-representation on this basis, and over $\mathbb{C}_{\mathrm{v}}$ this representation decomposes into simple modules. However, most simple modules are not $\mathbb{N}_{[\mathrm{v}]}^{0}$-representations and the decomposition can usually not be obtained via base change matrices with entries from $\mathbb{N}_{[\mathrm{v}]}^{0}$.

Secondly, even if $\mathrm{M} \cong \mathrm{M}^{\prime}$ are two isomorphic $\mathbb{N}_{[\mathrm{v}]^{0}}^{0}$-representations, they may not be $\mathbb{N}_{[\mathrm{v}]^{-}}^{0}$ equivalent. For example, the dihedral Hecke algebra of type $\mathrm{I}_{2}(12)$ has two $\mathbb{N}_{[\mathrm{v}]}^{0}$-representations, associated to the type $\mathbb{E}_{6}$ Dynkin, which are isomorphic over $\mathbb{C}_{\mathrm{v}}$ but not $\mathbb{N}_{[\mathrm{v}}^{0}$-equivalent (c.f. [MT16, Theorem II(iii)]).

Cells. For any $\mathbb{N}_{[\mathrm{v}]}^{0}$-algebra $\mathrm{P}$ one can define cell theory as in Definition 3.10, e.g. $\mathrm{x} \geq_{\mathrm{L}} \mathrm{y}$ for $\mathrm{x}, \mathrm{y} \in \mathrm{B}^{\mathrm{P}}$ if there exists an element $\mathrm{z} \in \mathrm{B}^{\mathrm{P}}$ such that $\mathrm{x}$ appears as a summand of $\mathrm{zy}$, when the latter is written as a linear combination of elements in $\mathrm{B}^{\mathrm{P}}$. We hence obtain (left, right and two-sided) cells $L, R$ and J, and we can write $L^{\prime} \geq_{L} L$ etc. See also e.g. [KM16] (incorporating v) for details. The same notions be can defined for any $\mathbb{N}_{[\mathrm{v}]}^{0}$-representation $\mathrm{M}$, e.g. $\mathrm{m} \geq_{\mathrm{L}} \mathrm{n}$ for $\mathrm{m}, \mathrm{n} \in \mathrm{B}^{\mathrm{M}}$ if there exists some $\mathrm{z} \in \mathrm{B}^{\mathrm{P}}$ such that $\mathrm{m}$ appears in $\mathrm{M}(\mathrm{z}) \mathrm{n}$ with non-zero coefficient when written in terms of $\mathrm{B}^{\mathrm{M}}$.

Definition 5.6. We call a $\mathbb{N}_{[\mathrm{v}]}^{0}$-representation $M$ transitive if all basis elements belong to the same $\sim_{\mathrm{L}}$ equivalence class.

Remark 5.7. Consider the graph with vertices given by $\mathrm{B}^{\mathrm{M}}$ and with an oriented edge from $\mathrm{n}$ to $\mathrm{m}$ whenever $\mathrm{m} \geq_{\mathrm{L}} \mathrm{n}$. Transitivity of $\mathrm{M}$ means that this graph is strongly connected.

Similarly, we can also define the notion of a transitive $\mathbb{Z}_{[\mathrm{v}]}$-representation associated to a strongly connected graph. (Note that $\mathrm{m} \geq_{\mathrm{L}} \mathrm{n}$ also makes sense over $\mathbb{Z}_{[\mathrm{v}]}$.) 
Definition 5.8. Fix L. Let $M\left(\geq_{L}\right)$, respectively $M\left(>_{L}\right)$, be the $\mathbb{N}_{[\mathrm{v}]}^{0}$-representations spanned by all $x \in \mathrm{B}^{\mathrm{P}}$ in the union of all left cells $\mathrm{L}^{\prime} \geq_{\mathrm{L}} \mathrm{L}$, respectively $\mathrm{L}^{\prime}>_{\mathrm{L}} \mathrm{L}$. (These are well-defined by [KM16, Proposition 1].) We call $\mathrm{C}_{\mathrm{L}}=\mathrm{M}\left(\mathrm{Z}_{\mathrm{L}}\right) / \mathrm{M}\left(>_{\mathrm{L}}\right)$ the (left) cell module for $\mathrm{L}$.

By definition, all cell modules are transitive $\mathbb{N}_{[\mathrm{v}]}^{0}$-representations.

Example 5.9. Coming back to Example 5.5: There is only one left (right, two-sided) cell for the group algebra of a finite group. The associated cell module is the regular representation.

However, on a different basis this might change considerably: The Hecke algebras for (finite) Coxeter groups are $\mathbb{N}_{[\mathrm{v}}^{0}$-algebras, where the KL basis plays the role of the basis $\mathrm{B}^{\mathrm{P}}$, see [KL79]. Their cell modules are Kazhdan-Lusztig's original cell modules. In the case of the symmetric group, these cell modules are the simple modules, but in general cell modules are not simple (since most simples are not $\mathbb{N}_{[\mathrm{v}]}^{0}$-representations).

Example 5.10. Decategorifications of cell 2-representations, which will be recalled below, are key examples of cell modules.

Given any cell module $\mathrm{C}_{\mathrm{L}}$, the results in [KM16, Section 8] show that there exists a unique, maximal two-sided cell, called apex, which does not annihilate $\mathrm{C}_{\mathrm{L}}$. The same is true for general transitive $\mathbb{N}_{[\mathrm{v}]}^{0}$-representations by [KM16, Section 9.2]. Thus, we can restrict the study of transitive $\mathbb{N}_{[\mathrm{v}]}^{0}$-representations to a given apex.

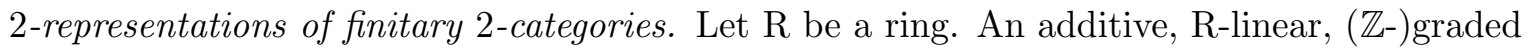
2-category $\mathscr{C}$ (with the same grading conventions as in Convention 4.3), which is idempotent complete and Krull-Schmidt, is called graded finitary if:

- It has finitely many objects, and all identity 1-morphisms are indecomposable.

- The 2-hom spaces are free of finite R-rank in each degree, and their grading is bounded from below.

- Consider the 2-subcategory of $\mathscr{C}$ having the same objects and 1-morphisms, but only degree-preserving 2-morphisms. Its split Grothendieck group is a free $\mathbb{Z}_{[\mathrm{v}]}$-module, with $\mathrm{v}$ corresponding to the grading shift, which we assume to be of finite $\mathbb{Z}_{[\mathrm{v}]}-$ rank.

Similarly, a graded locally finitary 2-category is as above, but relaxing the condition on the Grothendieck group by requiring it to be of countable $\mathbb{Z}_{[\mathrm{v}]}$-rank.

We also use graded finitary categories (having graded hom-spaces which are free of finite R-rank), which are the objects of a 2-category $\mathscr{A}_{\mathrm{gr}}^{\mathrm{f}}$ with 1-morphisms being additive, R-linear, degree-preserving functors and 2-morphisms being homogeneous natural transformations of degree-zero.

Let $\left(\mathscr{A}_{\mathrm{gr}}^{\mathrm{f}}\right)^{\star}$ denote the 2-category obtained from $\mathscr{A}_{\mathrm{gr}}^{\mathrm{f}}$ by adding formal shifts to the 1 -morphisms. Its 2 -hom spaces are given by

$$
\mathscr{H} \operatorname{om}_{\left(\mathscr{A g}_{\mathrm{gr}}^{\mathrm{f}}\right)^{\star}}(\mathrm{i}, \mathrm{j})=\bigoplus_{s \in \mathbb{Z}} \mathscr{H} \mathrm{om}_{\mathscr{A g}_{\mathrm{gr}}^{\mathrm{f}}}(\mathrm{i}\{s\}, \mathrm{j}) .
$$

Example 5.11. All 2-categories in Section 4 become graded (locally) finitary after taking their Karoubi envelope.

Example 5.12. Let B be a graded R-algebra which is free of finite R-rank. The category of free, finite R-rank, graded (left) B-representations is a prototypical object of $\mathscr{A}_{\mathrm{gr}}^{\mathrm{f}}$. For example, the graded representation categories of the quiver algebras $\nabla_{e}$ in Section $5 \mathrm{C}$ below are objects of $\mathscr{A}_{\mathrm{gr}}^{\mathrm{f}}$.

A graded finitary 2-representation of $\mathscr{C}$ is an additive, R-linear 2-functor

$$
\mathscr{M}: \mathscr{C} \rightarrow\left(\mathscr{A}_{\mathrm{gr}}^{\mathrm{f}}\right)^{\star}
$$

which is degree-preserving and commutes with shifts as in [MT16, Definition 3.4]. 
Example 5.13. The principal 2-representation $\mathscr{P}_{i}=\mathscr{C}\left(i,{ }_{-}\right)$, where $i$ is an object of $\mathscr{C}$, is a graded finitary 2-representation of $\mathscr{C}$.

Graded finitary 2-representations of $\mathscr{C}$ form a graded 2-category (in the sense of Convention 4.3), see [MM16a] for details, which can be adapted to the graded setting. In particular, there exists a well-defined notion of equivalence between such 2-representations.

For simplicity, we say 2-representation instead of graded finitary 2-representation etc. from now on, i.e. we omit the graded.

2-cells. As in the case of $\mathbb{N}_{[\mathrm{v}]}^{0}$-algebras, one can define cells and cell 2-representations of finitary 2-categories: Let $\mathrm{X}$ and $\mathrm{Y}$ be indecomposable 1-morphisms in a finitary 2-category $\mathscr{C}$. Set $\mathrm{X} \geq_{\mathrm{L}} \mathrm{Y}$ if $\mathrm{X}$ is isomorphic to a direct summand of $\mathrm{ZY}$, up to a degree shift, for some indecomposable 1-morphism Z. Similarly one defines $\geq_{R}$ and $\geq_{J}$. The equivalence classes for these are called the respective cells, denoted by L, R or J. All these notions can be defined in a similar way for 2-representations as well.

A finitary 2-representation $\mathscr{M}$ is transitive (see [MM16b, Section 3.1], or [MT16, Definition 3.6] in the graded setup), if $\mathscr{M}$ is supported on one $i \in \mathscr{C}$, and if all indecomposable objects $0, \mathrm{P} \in \mathscr{M}(\mathrm{i})$ are in the same $\sim_{\mathrm{L}}$-equivalence class. A transitive 2-representation is simple transitive, see [MM16b, Section 3.5] (or [MT16, Definition 3.6] in the graded setup), if it does not have any non-zero, proper $\mathscr{C}$-invariant ideals.

Remark 5.14. By [MM16b, Section 4], any 2-representation has a weak Jordan-Hölder series with simple transitive subquotients, which are unique up to permutation and equivalence. Therefore, it is natural to ask for the classification of simple transitive 2-representations.

Every (graded) finitary 2-category comes with a natural class of simple transitive 2representations:

Definition 5.15. Fix L. Then there exists $i \in \mathscr{C}$ such that all 1 -morphisms in $\mathrm{L}$ start at $i$. Let $\mathscr{M}\left(\mathrm{Z}_{\mathrm{L}}\right)$ be the 2-representations of $\mathscr{C}$ spanned by the additive closure of all indecomposable 1-morphisms $F$, in $\coprod_{j \in \mathscr{C}} \mathscr{P}_{i}(j)$, which belong to the union of all left cells $\mathrm{L}^{\prime} \geq_{\mathrm{L}} \mathrm{L}$. Let $\mathscr{Z}\left(\geq_{\mathrm{L}}\right)$ be the unique, proper two-sided 2-ideal in $\mathscr{M}\left(\geq_{\mathrm{L}}\right)$. (All of this is well-defined by [MM16b, Section 3.3 and Lemma 3].) We call $\mathscr{C}_{\mathrm{L}}=\mathscr{M}\left(\geq_{\mathrm{L}}\right) / \mathscr{Z}\left(\geq_{\mathrm{L}}\right)$ the cell 2-representation for $\mathrm{L}$.

Note that cell 2-representations are always simple transitive.

Example 5.16. In case of Soergel bimodules for the symmetric group, these exhaust all simple transitive 2-representations and categorify the simple modules [MM16b]. However, both these facts are false in general, as the example of dihedral Soergel bimodules shows, see e.g. [KMMZ16], [MT16].

Remark 5.17. On the decategorified level, the cell representation is obtained as the quotient of $\mathrm{M}\left(\geq_{\mathrm{L}}\right)$ by $\mathrm{M}\left(>_{\mathrm{L}}\right)$, cf. Definition 5.8. On the level of 2-representations, the proper maximal two-sided 2-ideal $\mathscr{Z}\left(\geq_{\mathrm{L}}\right)$ strictly contains the two-sided 2-ideal generated by the 2-subrepresentation $\mathscr{M}\left(>_{\mathrm{L}}\right)$ in general.

Again, there is a unique, maximal two-sided cell, called 2-apex, which does not annihilate a given cell 2-representation. The same works for general transitive 2-representations. See [CM16, Section 3.2] for more details.

(Co)algebra 1-morphisms. An algebra 1-morphism in $\mathscr{C}$ is a triple $(\mathrm{A}, \mu, \eta)$, where $\mathrm{A}$ is a 1-morphism and $\mu: \mathrm{AA} \rightarrow \mathrm{A}$ and $\eta: \mathbb{1} \rightarrow \mathrm{A}$ are 2-morphisms satisfying the usual axioms for the multiplication and unit of an algebra.

Furthermore, there are compatible notions of module 1-morphism over an algebra 1morphism A, and of 2-homomorphism between these. In this way, we get the 2-categories 
$\mathscr{M}_{\mathrm{od}_{\mathscr{C}}}$ (A) (or (A) $\mathscr{M}_{\mathrm{od}_{\mathscr{C}}}$ ) of right (or left) A-module 1-morphisms in $\mathscr{C}$. By post-composition $\mathscr{M}_{\operatorname{od}_{\mathscr{C}}}(\mathrm{A})$ becomes a left 2-representation of $\mathscr{C}$. Similarly, by pre-composition (A) $\mathscr{M}_{\text {od }}$ becomes a right 2-representation of $\mathscr{C}$.

One defines coalgebra 1-morphisms $(\mathrm{C}, \delta, \varepsilon)$ in $\mathscr{C}$ and their respective comodule 2-categories, which are also 2-representations of $\mathscr{C}$, dually.

Finally, there are also compatible notions of bimodule 1-morphism over an algebra 1morphism and 2-homomorphism between bimodule 1-morphisms. By definition, a Frobenius 1-morphism $\mathrm{F}$ in $\mathscr{C}$ is an algebra 1-morphism which is also a coalgebra 1-morphism, such that the comultiplication 2-morphism is a 2-homomorphism between $\mathrm{F}$-F-bimodule 1-morphisms.

We refer to [MMMT16] or [EGNO15, Chapter 7] for further details.

Remark 5.18. Suppose that $\mathscr{C}$ is additionally fiat (meaning that it has a certain involution [MM11, Section 2.4]). Then [MMMT16, Theorem 9] asserts that, for any simple transitive 2-representation $\mathscr{M}$ of $\mathscr{C}$, there exists a simple algebra 1-morphism $\mathrm{A}$ in $\overline{\mathscr{C}}$ (the projective abelianization of $\mathscr{C}$, as introduced in [MMMT16, Section 3.2]) such that $\mathscr{M}$ is equivalent (as a 2-representation of $\mathscr{C}$ ) to the subcategory of projective objects of $\mathscr{M}_{\text {od }}(\mathrm{A})$. Hence, the classification of simple transitive 2-representations of $\mathscr{C}$ is equivalent to the classification of simple algebra 1-morphisms in $\overline{\mathscr{C}}$. Or, dually, to the classification of cosimple coalgebra 1-morphisms in $\underline{\mathscr{C}}$, the injective abelianization of $\mathscr{C}$.

The fiat 2-categories $\mathscr{C}$ in this paper are special, because they are closely related to semisimple 2-categories by the quantum Satake correspondence, and the simple algebra 1-morphisms in this paper all belong to $\mathscr{C}$.

\section{A. Decategorified story.}

Trihedral transitive $\mathbb{N}_{[\mathrm{v}]}^{0}$-representations. From Sections $3 \mathrm{~B}$ and $3 \mathrm{C}$, in particular the connection to the representation theory of $\mathfrak{s l}_{3}$, the following is evident.

Proposition 5.19. The trihedral Hecke algebras are $\mathbb{N}_{[\mathrm{v}]}^{0}$-algebras, i.e. for the basis $\mathrm{C}^{\infty}$ and $\mathrm{C}^{e}$ from Propositions 3.9 an 3.14 we have

$$
\mathrm{xy} \in \mathbb{N}_{[\mathrm{v}]}^{0} \mathrm{C}^{\infty} \text { and } \mathrm{x}^{\prime} \mathrm{y}^{\prime} \in \mathbb{N}_{[\mathrm{v}]}^{0} \mathrm{C}^{e}
$$

for all $\mathrm{x}, \mathrm{y} \in \mathrm{C}^{\infty}$ and $\mathrm{x}^{\prime}, \mathrm{y}^{\prime} \in \mathrm{C}^{e}$. The same holds for the left colored KL bases.

This is our starting point for studying $\mathbb{N}_{[\mathrm{v}}^{0}$-representations of the trihedral Hecke algebras. From now on, we fix the right colored KL bases for $\mathrm{T}_{\infty}$ and $\mathrm{T}_{e}$, as in Proposition 5.19.

Example 5.20. Most of the three-dimensional $\mathrm{T}_{e}$-representations in (3-19) are not $\mathbb{N}_{[\mathrm{v}]}^{0}-$ representations (for any choice of basis).

For $e>1$, the one-dimensional representations $\mathrm{M}_{[3]_{\mathrm{v}} !, 0,0}, \mathrm{M}_{0,[3]_{\mathrm{v}} !, 0}$ and $\mathrm{M}_{0,0,[3]_{\mathrm{v}} !}$ are also not $\mathbb{N}_{[\mathrm{v}]}^{0}$-representations, e.g. by Example 3.6, the action of $\mathrm{c}_{g}^{1,1}$ on $\mathrm{M}_{[3]_{\mathrm{v}} !, 0,0}$ is given by

$$
\mathrm{c}_{g}^{1,1}=[2]_{\mathrm{v}}^{-2} \theta_{g} \theta_{p} \theta_{g}-\theta_{g} \mapsto-[3]_{\mathrm{v}} ! .
$$

Thus, $\mathrm{M}_{[3]_{\mathrm{v}} !, 0,0}$ is not an $\mathbb{N}_{[\mathrm{v}]}^{0}$-representation.

Our next goal is to define several families of $\mathbb{N}_{[\mathrm{v}}^{0}$-representations of the trihedral Hecke algebras. Recall from Proposition 3.11 and Corollary 3.15 that the trihedral algebras have one trivial and one non-trivial two-sided cell, both of which can be the apex of a transitive $\mathbb{N}_{[\mathrm{v}]}^{0}$-representation. For the trivial cell there is only one such representation:

Example 5.21. The simple $\mathrm{M}_{0,0,0}$ (cf. (3-19)) is a transitive $\mathbb{N}_{[\mathrm{v}]}^{0}$-representation of $\mathrm{T}_{\infty}$, which also descends to $\mathrm{T}_{e}$ for any $e$, and its apex is the trivial cell. By (3-16) and Example 5.20, there are no other transitive $\mathbb{N}_{[\mathrm{v}]}^{0}$-representations whose apex is the trivial cell. 
From now on we will only consider transitive $\mathbb{N}_{[\mathrm{v} \mid}^{0}$-representations whose apex is the unique, non-trivial two-sided cell. For this purpose, we consider tricolored graphs, denoted by $\boldsymbol{\Gamma}$ etc., fixing certain conventions as follows.

Graph-theoretic recollections. For us a graph $\boldsymbol{\Gamma}$ is an undirected, connected, finite graph without loops, but possibly with multiple edges. We will also need graphs with directed edges and we indicate these by adding the superscript ${ }_{-}^{\mathrm{X}}$ or $_{-}{ }^{\mathrm{Y}}$.

We call $\boldsymbol{\Gamma}=(\boldsymbol{\Gamma}, V=\{G, O, P\}, E=\{B, R, Y\})$ tricolored, with colors $g, o, p$, if $V$ and $E$ can be partitioned into three disjoint sets $G, O, P$ and $B, R, Y$ such that

$$
\left(\begin{array}{c}
\bullet \sim \in Y \Rightarrow \\
\bullet \in G \text { and } \square \in O,
\end{array}\right) \quad\left(\begin{array}{c}
\bullet-\cdots \in R \Rightarrow \\
\square \in O \text { and } \bullet \in P,
\end{array}\right) \quad\left(\begin{array}{c}
\bullet \cdots \cdots \cdots \cdots \in B \Rightarrow \\
\bullet \in P \text { and } \bullet \in G .
\end{array}\right)
$$

(We usually denote a tricolored graph simply by $\boldsymbol{\Gamma}$, suppressing the tricoloring.)

The vertices of any tricolored graph $\boldsymbol{\Gamma}$ can be ordered such that the adjacency matrix $A(\boldsymbol{\Gamma})$ is of the following form.

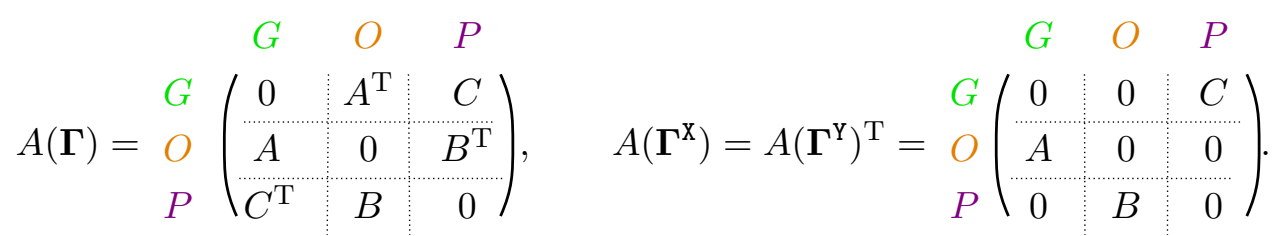

Here $A, B, C$ are matrices with entries in $\mathbb{N}^{0}$, encoding the connections $G \rightarrow O$ (matrix $A$ ), $O \rightarrow P$ (matrix $B$ ), and $P \rightarrow G$ (matrix $C$ ). We will always consider vertex-orderings of this form. Moreover, $\boldsymbol{\Gamma}$ has two associated directed graphs $\Gamma^{\mathrm{X}}$ and $\boldsymbol{\Gamma}^{\mathrm{Y}}$ whose adjacency matrices are $A\left(\boldsymbol{\Gamma}^{\mathrm{X}}\right)$ and $A\left(\boldsymbol{\Gamma}^{\mathrm{Y}}\right)$ as in (5-1). They have the same vertex sets as $\boldsymbol{\Gamma}$, but their edges are oriented according to (3-1).

We write $i \in \boldsymbol{\Gamma}$ ( $i \in G$ etc.) meaning that $i$ is a (g-colored etc.) vertex of $\boldsymbol{\Gamma}$. Furthermore, we denote by $S_{\boldsymbol{\Gamma}}$ the spectrum of $\boldsymbol{\Gamma}$, i.e. the multiset of eigenvalues of $A(\boldsymbol{\Gamma})$, and we use similar notations for $\Gamma^{\mathrm{X}}$ and $\Gamma^{\mathrm{Y}}$.

Example 5.22. Our main examples of tricolored graphs are all displayed in Section AppA. Their spectra play an important role for us.

Example 5.23. The simplest examples, which are, however, fundamental for this paper, are the generalized type A Dynkin diagrams, e.g.:

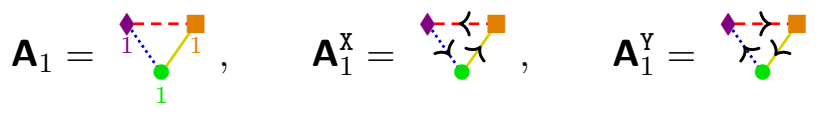

$$
\begin{aligned}
& A=(1) \quad B=(1) \quad C=(1)
\end{aligned}
$$

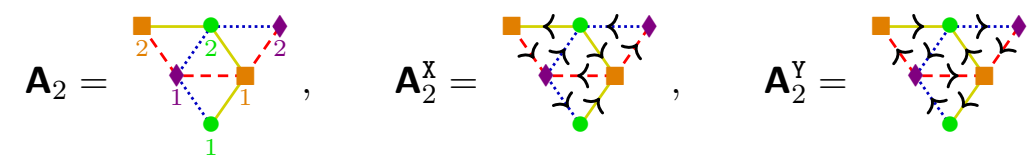

$$
\begin{aligned}
& A=\left(\begin{array}{ll}
1 & 1 \\
0 & 1
\end{array}\right) \quad B=\left(\begin{array}{ll}
1 & 1 \\
1 & 0
\end{array}\right) \quad C=\left(\begin{array}{ll}
1 & 0 \\
1 & 1
\end{array}\right) \\
& \mathbf{A}_{3}=
\end{aligned}
$$

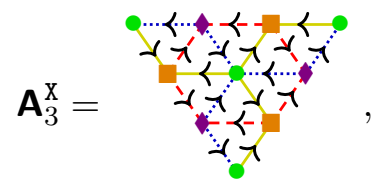

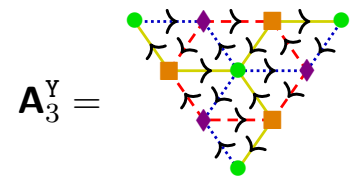




$$
A=\left(\begin{array}{llll}
1 & 1 & 0 & 0 \\
0 & 1 & 0 & 1 \\
0 & 1 & 1 & 0
\end{array}\right) \quad B=\left(\begin{array}{ccc}
1 & 1 & 0 \\
1 & 0 & 1 \\
0 & 1 & 1
\end{array}\right) \quad C=\left(\begin{array}{lll}
1 & 0 & 0 \\
1 & 1 & 1 \\
0 & 1 & 0 \\
0 & 0 & 1
\end{array}\right)
$$

(The matrices $A, B, C$ are given with respect to the ordering of the vertices as indicated in the unoriented graphs.) The vertices of these graphs can be identified with the cut-offs of the positive Weyl chamber of $\mathfrak{s l}_{3}$, cf. (2-1), where e.g the vertex with label 4 in $\mathbf{A}_{3}$ corresponds to the $\mathfrak{s l}_{3}$-weight $(0,3)$.

Moreover, the spectra of these graphs are:

$$
\begin{gathered}
S_{\mathbf{A}_{1}^{\mathrm{x}}}=\left\{\operatorname{roots} \text { of }(X-1)\left(X^{2}+X+1\right)\right\}, \\
S_{\mathbf{A}_{2}^{\mathrm{X}}}=\left\{\text { roots of }\left(X^{2}-X-1\right)\left(X^{4}+X^{3}+2 X^{2}-X+1\right)\right\}, \\
S_{\mathbf{A}_{3}^{\mathrm{X}}}=\left\{\operatorname{roots} \text { of } X(X-2)\left(X^{2}+2 X+4\right)\left(X^{6}-X^{3}+1\right)\right\} .
\end{gathered}
$$

The reader should compare these to Example 2.12.

Next, recall that an oriented graph $\boldsymbol{\Gamma}^{\text {or }}$ is called strongly connected, if there is a path from $i$ to $j$ for any $i, j \in \Gamma^{\text {or }}$, and it is called weakly regular if the numbers of incoming and outgoing edges agree at each vertex, counting $r$ parallel edges $r^{2}$ times (e.g. a vertex with two incoming parallel edges must have two outgoing parallel edges or four outgoing single edges). By convention, we call $\boldsymbol{\Gamma}$ as above strongly connected, respectively weakly regular, if $\Gamma^{\mathrm{X}}$ and $\Gamma^{\mathrm{Y}}$ are both strongly connected, respectively weakly regular.

Definition 5.24. A graph $\Gamma$ is called admissible if it admits a tricoloring, such that $\Gamma$ is strongly connected and weakly regular.

Example 5.25. All of our main examples from Section AppA are admissible.

Lemma 5.26. The matrices $A, B, C$ in (5-1), which are blocks of $A(\boldsymbol{\Gamma})$, satisfy

$$
A^{\mathrm{T}} A=C C^{\mathrm{T}}, \quad A A^{\mathrm{T}}=B^{\mathrm{T}} B, \quad C^{\mathrm{T}} C=B B^{\mathrm{T}}
$$

if and only if $\boldsymbol{\Gamma}$ is weakly regular.

In particular, $A A^{\mathrm{T}}, A^{\mathrm{T}} A, B B^{\mathrm{T}}, B^{\mathrm{T}} B, C C^{\mathrm{T}}$ and $C^{\mathrm{T}} C$ have the same non-zero eigenvalues for any weakly regular graph $\boldsymbol{\Gamma}$.

Proof. By symmetry under color exchange, it suffices to prove $A^{\mathrm{T}} A=C C^{\mathrm{T}}$. In the graph sum $\Gamma^{\mathrm{X}}+\boldsymbol{\Gamma}^{\mathrm{Y}}$, the entries of $A^{\mathrm{T}} A$ count the number of two-step paths $G \rightarrow O \rightarrow G$, while the entries of $C C^{\mathrm{T}}$ count the number of two-step paths $G \rightarrow P \rightarrow G$. Thus, $A^{\mathrm{T}} A=C C^{\mathrm{T}}$ if and only if, for all $i \in G$, the number of incoming edges (i.e. those coming from vertices in $P$ ) agrees with the number of outgoing edges (i.e. those going to vertices in $O$ ) in $\Gamma^{\mathbb{X}}$ (there is an equivalent statement for $\Gamma^{\mathrm{Y}}$, of course), where we need to count $r$ parallel edges $r^{2}$ times.

Some trihedral $\mathbb{N}_{[\mathrm{v}]}^{0}$-represenations. We denote by $\mathbb{C}_{\mathrm{v}}\{G, O, P\}$ the free $\left(\mathbb{C}_{\mathrm{v}^{-}}\right)$vector space on the vertex set of $\boldsymbol{\Gamma}$.

Definition 5.27. We define a $\mathrm{T}_{\infty}$-representation

$$
\mathrm{M}_{\boldsymbol{\Gamma}}: \mathrm{T}_{\infty} \rightarrow \operatorname{End}_{\mathbb{C}_{\mathrm{v}}}\left(\mathbb{C}_{\mathrm{v}}\{G, O, P\}\right)
$$

by associating the following matrices to the generators $\theta_{g}, \theta_{o}, \theta_{p}$ :

$$
\begin{gathered}
\mathrm{M}_{\Gamma}\left(\theta_{g}\right)=[2]_{\mathrm{v}}\left(\begin{array}{ccc}
{[3]_{\mathrm{v}} \mathrm{Id}} & A^{\mathrm{T}} & C \\
0 & 0 & 0 \\
0 & 0 & 0
\end{array}\right), \quad \mathrm{M}_{\boldsymbol{\Gamma}}\left(\theta_{o}\right)=[2]_{\mathrm{v}}\left(\begin{array}{ccc}
0 & 0 & 0 \\
A & {[3]_{\mathrm{v}} \mathrm{Id}} & B^{\mathrm{T}} \\
0 & 0 & 0
\end{array}\right) \\
\mathrm{M}_{\boldsymbol{\Gamma}}\left(\theta_{p}\right)=[2]_{\mathrm{v}}\left(\begin{array}{ccc}
0 & 0 & 0 \\
0 & 0 & 0 \\
C^{\mathrm{T}} & B & {[3]_{\mathrm{v}} \mathrm{Id}}
\end{array}\right) .
\end{gathered}
$$

Here $A, B, C$ are as in (5-1). 
Note that we have

$$
\mathrm{M}_{\boldsymbol{\Gamma}}^{\text {tot }}=\mathrm{M}_{\boldsymbol{\Gamma}}\left(\theta_{g}\right)+\mathrm{M}_{\boldsymbol{\Gamma}}\left(\theta_{o}\right)+\mathrm{M}_{\boldsymbol{\Gamma}}\left(\theta_{p}\right)=[2]_{\mathrm{v}}\left([3]_{\mathrm{v}} \mathrm{Id}+A(\boldsymbol{\Gamma})\right) .
$$

Remark 5.28. The three-dimensional simple $T_{e}$-representations $\mathrm{M}_{z}$ in (3-17) are similar to the $\mathrm{M}_{\boldsymbol{\Gamma}}$ in (5-3). In $\mathrm{M}_{\boldsymbol{\Gamma}}$ the complex entry $z$ of $\mathrm{M}_{z}$ has been replaced by $\mathbb{N}^{0}$-matrices $A, B, C$ which have these complex numbers as eigenvalues, as we will see in Corollary 5.36 below. However, in $\mathrm{M}_{\boldsymbol{\Gamma}}$ the matrices $A, B, C$ need not be equal, whereas in $\mathrm{M}_{z}$ we only have one complex number.

We always choose $\{G, O, P\}$ as a basis. Recalling the setup from Section 5A we get:

Lemma 5.29. $\mathrm{M}_{\boldsymbol{\Gamma}}$ is well-defined if and only if $\boldsymbol{\Gamma}$ is weakly regular.

Proof. By direct computation, one immediately sees that (3-3) always holds, irrespective of $A, B$ and $C$. Furthermore, note that $\mathrm{M}_{\boldsymbol{\Gamma}}$ preserves the relations in (3-4) if and only if the equations in (5-2) hold. The claim then follows from Lemma 5.26.

From now on we assume that $\boldsymbol{\Gamma}$ is weakly regular whenever we write $\mathrm{M}_{\boldsymbol{\Gamma}}$. Proving that these are $\mathbb{N}_{[\mathrm{v}]}^{0}$-representations is hard and follows from categorification. However, if we drop the positivity condition, then the following is clear by noting that the scalars $[2]_{\mathrm{v}}^{-k-l}$ appearing in the definition of the colored KL elements cancel against the positive powers of $[2]_{\mathrm{v}}$ in (5-3).

Lemma 5.30. $\mathrm{M}_{\Gamma}$ is a transitive $\mathbb{Z}_{[\mathrm{v}]}$-representation if and only if $\boldsymbol{\Gamma}$ is admissible.

Example 5.31. Take $e=2$ and the graph $\mathbf{A}_{2}$ as in Section AppA. Fix $g$ as a starting color. Then the six non-trivial, colored KL basis elements of $\mathrm{T}_{2}$ act on $\mathrm{M}_{\mathbf{A}_{2}}$ via matrices whose entries are all in $\mathbb{N}_{[\mathrm{v}]}^{0}$. For $\mathrm{c}_{g}^{0,0}=\theta_{g}, \mathrm{c}_{g}^{1,0}=[2]_{\mathrm{v}}^{-1} \theta_{o} \theta_{g}$ and $\mathrm{c}_{g}^{0,1}=[2]_{\mathrm{v}}^{-1} \theta_{p} \theta_{g}$ this is immediately clear. For the other basis elements, one can check the claim by calculation. For example, $\mathrm{c}_{g}^{2,0}=[2]_{\mathrm{v}}^{-2} \theta_{g} \theta_{o} \theta_{g}-[2]_{\mathrm{v}}^{-1} \theta_{p} \theta_{g}$, since $\mathrm{U}_{2,0}(\mathrm{X}, \mathrm{Y})=\mathrm{X}^{2}-\mathrm{Y}$, so

$$
\mathrm{M}_{\mathbf{A}_{2}}\left(c_{g}^{2,0}\right)=[2]_{\mathrm{v}}\left(\begin{array}{cccccc}
0 & 0 & 0 & 0 & 0 & 0 \\
0 & 0 & 0 & 0 & 0 & 0 \\
0 & 0 & 0 & 0 & 0 & 0 \\
0 & 0 & 0 & 0 & 0 & 0 \\
0 & {[3]_{\mathrm{v}}} & 1 & 1 & 1 & 1 \\
{[3]_{\mathrm{v}}} & 0 & 1 & 0 & 1 & 0
\end{array}\right) .
$$

The matrices associated to $\mathrm{c}_{g}^{1,1}$ and $\mathrm{c}_{g}^{0,2}$ can be computed similarly. The fact that we get a $\mathbb{N}_{[\mathrm{v}]}^{0}$-representation is non-trivial, because the expressions for the $\mathrm{c}_{g}^{m, n}$ in terms of the $\mathrm{h}_{\mathbf{u}}^{k, l}$ have negative coefficients.

The following can be proved as in the dihedral case [MT16, Section 5.4].

Lemma 5.32. Let $\boldsymbol{\Gamma}$ and $\boldsymbol{\Gamma}^{\prime}$ be two admissible graphs. Then $\mathrm{M}_{\boldsymbol{\Gamma}} \cong_{+} \mathrm{M}_{\boldsymbol{\Gamma}^{\prime}}$ if and only if $\boldsymbol{\Gamma}$ and $\boldsymbol{\Gamma}^{\prime}$ are isomorphic as tricolored graphs.

Lemma 5.33. Let $M$ be a transitive $\mathbb{N}_{[\mathrm{v}]}^{0}$-representation of $\mathrm{T}_{\infty}$ which satisfies

$$
\mathrm{M}\left(\theta_{\mathbf{u}}\right) \mathrm{m}=a \mathrm{~m}+\mathbb{N}_{[\mathrm{v}]}^{0} \mathrm{~B}^{\mathrm{M}}-\{\mathrm{m}\} \Rightarrow a \in\left\{0,[3]_{\mathrm{v}} !\right\}, \quad \text { for all } \mathbf{u}, m_{j} .
$$

Then there exists an admissible graph $\Gamma$ with $\mathrm{M} \cong{ }_{+} \mathrm{M}_{\Gamma}$.

Proof. Recall that $\mathrm{M}$ has a fixed basis $\mathrm{B}^{\mathrm{M}}$ on which all elements of the colored KL basis act by matrices with entries in $\mathbb{N}_{[\mathrm{v}]}^{0}$. In particular, the assumption implies that for each generator $\theta_{\mathbf{u}}$ there is an ordering of $\mathrm{B}^{\mathrm{M}}$ such that

$$
\mathrm{M}\left(\theta_{\mathbf{u}}\right)=\left(\begin{array}{cc}
{[3]_{\mathrm{v}} ! \mathrm{Id}} & D \\
0 & 0
\end{array}\right)
$$

for some matrix $D$ with entries in $\mathbb{N}_{[\mathrm{v}]}^{0}$. The rest of the proof now follows along the lines of [Zim17, Corollary 5.5] or [KMMZ16, Section 4.3]: 
- First observe that each $\mathrm{m}$ is a $[3]_{\mathrm{v}}$ ! -eigenvector of some $\theta_{\mathbf{u}}$, since otherwise $\mathrm{M}\left(\theta_{g}\right)+$ $\mathrm{M}\left(\theta_{0}\right)+\mathrm{M}\left(\theta_{p}\right)$ would have a zero row by (5-4), which contradicts the transitivity.

- Secondly, $\mathrm{m}$ is not a $[3]_{\mathrm{v}}$ !-eigenvector for all the $\theta_{\mathbf{u}}$. To see this, assume the contrary. Then, by transitivity, $\mathrm{M}$ has to be one-dimensional with all $\theta_{\mathbf{u}}$ acting by $[3]_{\mathrm{v}}$ ! . However, as in Lemma 3.17, this contradicts the fact that $\mathrm{M}$ is a $\mathrm{T}_{e}$-representation.

- Finally, $\mathrm{m}$ is not a common $[3]_{\mathrm{v}}$ !-eigenvector of two of the $\theta_{\mathbf{u}}$. Assume on the contrary that $\theta_{g}$ and $\theta_{o}$ had such a common eigenvector. Then $\mathrm{M}\left(\theta_{g}\right) \mathrm{m}=\mathrm{M}\left(\theta_{o}\right) \mathrm{m}=[3]_{\mathrm{v}} ! \mathrm{m}$ and $\mathrm{M}\left(\theta_{p}\right) \mathrm{m}=0$. This contradicts $(3-4)$.

Together with Lemmas 5.26, 5.29 and 5.30, this proves the claim.

The classification problem $C P$ 5.34. Back to the polynomials $\mathrm{U}_{m, n}(\mathrm{X}, \mathrm{Y})$ from Definition 2.5. Observe that weak regularity implies that

$$
A\left(\boldsymbol{\Gamma}^{\mathrm{X}}\right) A\left(\boldsymbol{\Gamma}^{\mathrm{Y}}\right)=\left(\begin{array}{ccc}
C C^{\mathrm{T}} & 0 & 0 \\
0 & A A^{\mathrm{T}} & 0 \\
0 & 0 & B B^{\mathrm{T}}
\end{array}\right) \stackrel{(5-2)}{=}\left(\begin{array}{ccc}
A^{\mathrm{T}} A & 0 & 0 \\
0 & B^{\mathrm{T}} B & 0 \\
0 & 0 & C^{\mathrm{T}} C
\end{array}\right)=A\left(\boldsymbol{\Gamma}^{\mathrm{Y}}\right) A\left(\boldsymbol{\Gamma}^{\mathrm{X}}\right) .
$$

Thus, we can formulate the following classification problem.

CP 5.34. Classify all admissible graphs $\boldsymbol{\Gamma}$ such that

$$
\mathrm{U}_{m, n}\left(A\left(\boldsymbol{\Gamma}^{\mathrm{X}}\right), A\left(\boldsymbol{\Gamma}^{\mathrm{Y}}\right)\right)=0, \quad \text { for all } m+n=e+1 .
$$

(In other words, classify all admissible graphs $\boldsymbol{\Gamma}$ such that $z \in S_{\Gamma^{\mathrm{x}}}$ only if $(z, \bar{z}) \in \mathrm{V}_{e}$.)

Proposition 5.35. A graph $\Gamma$ is a solution of CP 5.34 if and only if $\mathrm{M}_{\boldsymbol{\Gamma}}$ descends to a transitive $\mathbb{Z}_{[\mathrm{v}]}$-representation of $\mathrm{T}_{e}$.

Proof. Recall that admissible graphs are always strongly connected. Thus, the claim about transitivity is clear and it remains to check the other claims.

To this end, fix $m, n$. Observe that $\mathrm{U}_{m, n}\left(A\left(\boldsymbol{\Gamma}^{\mathrm{X}}\right), A\left(\boldsymbol{\Gamma}^{\mathrm{Y}}\right)\right)$ has at most one non-zero block matrix entry in each of the $G$-, $O$ - and $P$-rows (as indicated in (5-1)), since $A\left(\boldsymbol{\Gamma}^{\mathrm{X}}\right)$ and $A\left(\boldsymbol{\Gamma}^{\mathrm{Y}}\right)$ just permute the $G$-, $O$ - and $P$-blocks, and multiply them by $A, B, C$ or their transpose. Let us denote these block matrix entries by $N_{m, n}^{G}, N_{m, n}^{O}$ and $N_{m, n}^{P}$.

Let us fix $g$ as a starting color, the other two cases work verbatim. In this case an easy calculation yields:

$$
\begin{aligned}
& \mathrm{M}_{\boldsymbol{\Gamma}}\left(\mathrm{c}_{g}^{m, n}\right)=[2]_{\mathrm{v}}\left(\begin{array}{ccc}
N_{m, n}^{G} \cdot[3]_{\mathrm{v}} \mathrm{Id} & N_{m, n}^{G} \cdot A^{\mathrm{T}} & N_{m, n}^{G} \cdot C \\
0 & 0 & 0 \\
0 & 0 & 0
\end{array}\right), \quad \text { if } m+2 n \equiv 0 \bmod 3, \\
& \mathrm{M}_{\boldsymbol{\Gamma}}\left(\mathrm{c}_{g}^{m, n}\right)=[2]_{\mathrm{v}}\left(\begin{array}{ccc}
0 & 0 & 0 \\
N_{m, n}^{O} \cdot[3]_{\mathrm{v}} \mathrm{Id} & N_{m, n}^{O} \cdot A^{\mathrm{T}} & N_{m, n}^{O} \cdot C \\
0 & 0 & 0
\end{array}\right), \quad \text { if } m+2 n \equiv 1 \bmod 3, \\
& \mathrm{M}_{\boldsymbol{\Gamma}}\left(\mathrm{c}_{g}^{m, n}\right)=[2]_{\mathrm{v}}\left(\begin{array}{ccc}
0 & 0 & 0 \\
0 & 0 & 0 \\
N_{m, n}^{P} \cdot[3]_{\mathrm{v}} \mathrm{Id} & N_{m, n}^{P} \cdot A^{\mathrm{T}} & N_{m, n}^{P} \cdot C
\end{array}\right), \quad \text { if } m+2 n \equiv 2 \bmod 3 .
\end{aligned}
$$

As in the proof of Lemma 3.18, we note that in the calculation of $\mathrm{M}_{\boldsymbol{\Gamma}}\left(\mathrm{c}_{g}^{m, n}\right)$ the positive powers of $[2]_{\mathrm{v}}$, due to (5-3), cancel against the negative powers of [2] $]_{\mathrm{v}}$, which appear in (3-10), up to an overall factor $[2]_{\mathrm{v}}$. We see that $\mathrm{M}_{\boldsymbol{\Gamma}}\left(\mathrm{c}_{g}^{m, n}\right)$ vanishes if and only if $\mathrm{U}_{m, n}\left(A\left(\boldsymbol{\Gamma}^{\mathrm{X}}\right), A\left(\boldsymbol{\Gamma}^{\mathrm{Y}}\right)\right)=0$.

Our main examples of solutions of CP 5.34 are the graphs from Section AppA. Indeed, as can be seen in Section AppB, their spectra are such that Proposition 5.35 applies: 
Corollary 5.36. The generalized ADE Dynkin diagrams from Section AppA give transitive $\mathbb{Z}_{[\mathrm{v}]}$-representations $\mathrm{M}_{\boldsymbol{\Gamma}}$ for the associated level $e$.

By Lemmas 5.32, 5.33 and Proposition 5.35, classifying all $\mathbb{Z}_{[\mathrm{v}]}$-representations of $\mathrm{T}_{e}$ boils down to CP 5.34. We have already seen that the generalized ADE Dynkin diagrams give solutions of CP 5.34. So two questions remain: whether these are all solutions and whether these are $\mathbb{N}_{[\mathrm{v} v}^{0}$-representations (transitivity is clear because the graphs are strongly connected).

We do not have a complete answer to these questions. However, we are able to prove:

Proposition 5.37. Let $g, o, p$ indicate the starting color. Then we have (at least) the following transitive $\mathbb{N}_{[\mathrm{v}]}^{0}$-representations of $\mathrm{T}_{e}$.

\begin{tabular}{r|c:c} 
& $e \equiv 0 \bmod 3$ & $e \neq \equiv \bmod 3$ \\
\hline $\mathbb{N}_{[\mathrm{v}]}^{0}$-reps. & $\mathrm{M}_{\mathbf{A}_{e}^{g}}, \mathrm{M}_{\mathbf{A}_{e}^{o}}, \mathrm{M}_{\mathbf{A}_{e}^{p}}$, & $\mathrm{M}_{\mathbf{A}_{e}^{g}}$ \\
quantity & $\mathrm{M}_{\mathbf{D}_{e}^{g}}, \mathrm{M}_{\mathbf{D}_{e}^{o}}, \mathrm{M}_{\mathbf{D}_{e}^{p}}$ & 1
\end{tabular}

Moreover, the representations $\mathrm{M}_{\mathbf{A}_{e}}$ are the cell modules of $\mathrm{T}_{e}$.

Proof. Except for the claim about positivity, this is clear by Corollary 5.36, Lemmas 5.30, 5.32 and the construction.

For example, if $e \not \equiv 0 \bmod 3$, then there is only one type $A$ representation up to $\mathbb{N}_{[\mathrm{v}]^{-}}^{0}$ equivalence, since all tricolorings of $\mathbf{A}_{e}$ give isomorphic tricolored graphs. To see this, note that a tricoloring of the lowest triangle fixes the tricoloring of the whole graph, and that there are six choices. When $e \not \equiv 0 \bmod 3$, they all give isomorphic tricolored graphs, as can be easily seen. When $e \equiv 0 \bmod 3$, we get three different isomorphism classes of tricolored graphs, which are determined by the color of the corner vertices. Note that there is one more vertex with that color, e.g. $g$, than vertices with one of the other two colors, e.g. 0 resp. $p$. This explains why tricolored graphs whose corner vertices have different colors, are non-isomorphic. Finally, for any fixed color of the corner vertices, there are two tricolored graphs, which are isomorphic by a $\mathbb{Z} / 2 \mathbb{Z}$ symmetry in the bisector of the angle at that vertex.

Positivity follows from Theorem 5.46 which we proof later on.

In contrast to simples, the transitive $\mathbb{N}_{[\mathrm{v}]}^{0}$-representations of $\mathrm{T}_{e}$ can get arbitrarily large as $e$ grows, c.f. (3-19) and (5-5). We only know their complete classification for small values of $e$.

Classification for small levels.

Theorem 5.38. Let $e \in\{0,1,2,3\}$. An admissible graph $\boldsymbol{\Gamma}$ provides a solution to CP 5.34 if and only if $\boldsymbol{\Gamma}$ is a generalized ADE Dynkin diagram of the corresponding level or

$$
\boldsymbol{\Gamma}=\mathbf{y}, \text { for } e=3,
$$

called special solution. (Note the double edges.)

Proof. We do the hardest case where $e=3$ and omit the others, all of which can be proven similarly. In this case, the vanishing ideal $\mathrm{J}_{3}$ is generated by

$$
\left\{\mathrm{U}_{4,0}(\mathrm{X}, \mathrm{Y}), \mathrm{U}_{3,1}(\mathrm{X}, \mathrm{Y}), \mathrm{U}_{2,2}(\mathrm{X}, \mathrm{Y}), \mathrm{U}_{1,3}(\mathrm{X}, \mathrm{Y}), \mathrm{U}_{0,4}(\mathrm{X}, \mathrm{Y})\right\} \subset \mathbb{Z}[\mathrm{X}, \mathrm{Y}]
$$

with the polynomials as in Example 2.6. We proceed as follows: consider the polynomials

$$
\left\{\mathrm{Y}^{4} \mathrm{U}_{4,0}(\mathrm{X}, \mathrm{Y}), \mathrm{Y}^{2} \mathrm{U}_{3,1}(\mathrm{X}, \mathrm{Y}), \mathrm{Y}^{3} \mathrm{U}_{2,2}(\mathrm{X}, \mathrm{Y}), \mathrm{YU}_{1,3}(\mathrm{X}, \mathrm{Y}), \mathrm{Y}^{2} \mathrm{U}_{0,4}(\mathrm{X}, \mathrm{Y})\right\} \subset \mathbb{Z}[\mathrm{X}, \mathrm{Y}],
$$

which are now polynomials in the variables $\mathrm{x}=\mathrm{XY}$ and $\mathrm{y}=\mathrm{Y}^{3}$. Clearly, any solution of CP 5.34 gives an admissible graph $\boldsymbol{\Gamma}$ such that $\left(\mathrm{x}=A\left(\boldsymbol{\Gamma}^{\mathrm{X}}\right) A\left(\boldsymbol{\Gamma}^{\mathrm{Y}}\right), \mathrm{y}=A\left(\boldsymbol{\Gamma}^{\mathrm{Y}}\right)^{3}\right)$ is annihilated 
by the polynomials in (5-7). Hence, one can solve CP 5.34 by first classifying all solutions of (5-7) in terms of $\mathrm{x}$ and $\mathrm{y}$ and then checking which ones give solutions of CP 5.34 in terms of $A\left(\boldsymbol{\Gamma}^{\mathrm{X}}\right)$ and $A\left(\boldsymbol{\Gamma}^{\mathrm{Y}}\right)$.

We can use the theory of Gröbner bases to rewrite (5-7). This shows that $\boldsymbol{\Gamma}$ solves CP 5.34 if and only if $\mathrm{x}$ and $\mathrm{y}$ satisfy

$$
\begin{gathered}
\left(\mathrm{x}^{3}-5 \mathrm{x}^{2}+4 \mathrm{x}=0 \quad \& \mathrm{xy}-\mathrm{y}-2 \mathrm{x}^{2}+2 \mathrm{x}=0 \quad \& \mathrm{y}^{2}-\mathrm{y}-5 \mathrm{x}^{2}+6 \mathrm{x}=0\right) \text { or } \\
\left(\mathrm{y}^{4}-9 \mathrm{y}^{3}+9 \mathrm{y}^{2}-8 \mathrm{y}=0 \& 38 \mathrm{x}-5 \mathrm{y}^{3}+43 \mathrm{y}^{2}-43 \mathrm{y}=0\right)
\end{gathered}
$$

We observe further that $A\left(\boldsymbol{\Gamma}^{\mathrm{X}}\right) A\left(\boldsymbol{\Gamma}^{\mathrm{Y}}\right)$ vanishes if and only if $A^{\mathrm{T}} A$ does, and similarly $A\left(\boldsymbol{\Gamma}^{\mathrm{Y}}\right)^{3}$ vanishes if and only if $C B A$ does. Thus, it suffices to solve (5-8) for $\mathrm{x}=A^{\mathrm{T}} A$ and $\mathrm{y}=C B A$. The upshot is that the first equation in (5-8) is then an equation in one symmetric matrix $A^{\mathrm{T}} A$ with entries from $\mathbb{N}^{0}$.

Note that the complex roots of the associated polynomial $\mathrm{x}^{3}-5 \mathrm{x}^{2}+4 \mathrm{x}=\mathrm{x}(\mathrm{x}-1)(\mathrm{x}-4)$ are $0,1,4$, and that $\left(\begin{array}{cc}0 & A^{\mathrm{T}} \\ A & 0\end{array}\right)$ is the adjacency matrix of the bicolored subgraph of $\boldsymbol{\Gamma}$ obtained by erasing $P$ (and all edges with a vertex in $P$ ). Therefore, $\left(\begin{array}{ll}0 & A^{\mathrm{T}} \\ A & 0\end{array}\right)$ has to be the adjacency matrix of an affine type ADE Dynkin diagram, by the classification in [Smi70], [BH12, Section 3.1.1], provided it is not zero. To see this, note that $A^{\mathrm{T}} A$ has to have the maximal eigenvalue 4 , since otherwise

$$
\mathrm{y}^{4}-9 \mathrm{y}^{3}+9 \mathrm{y}^{2}-8 \mathrm{y}=\mathrm{y}(\mathrm{y}-8)\left(\mathrm{y}^{2}-\mathrm{y}+1\right)=0
$$

cannot hold: if the maximal eigenvalue of $A^{\mathrm{T}} A$ were 1 , then the same would hold for $B^{\mathrm{T}} B$ and $C^{\mathrm{T}} C$ by (5-2), which makes it impossible for $C B A$ to satisfy (5-9). Hence, we obtain the following possibilities for the associated bipartite graph.

$$
\begin{aligned}
& \tilde{\mathrm{A}}_{1}=\Longleftrightarrow, \quad \tilde{\mathrm{A}}_{3}=\ldots, \quad \tilde{\mathrm{A}}_{5}=\longmapsto, \tilde{\mathrm{D}}_{4}^{G=1}= \\
& \tilde{\mathrm{D}}_{4}^{G=4}=\left\{, \quad \tilde{\mathrm{D}}_{5}=\left\{, \quad \tilde{\mathrm{E}}_{6}^{G=3}=\right.\right.
\end{aligned}
$$

\begin{tabular}{|c|c|c|c|c|c|c|c|c|c|}
\hline $\boldsymbol{\Gamma}_{g, o}$ & $\tilde{\mathrm{A}}_{1}$ & $\tilde{\mathrm{A}}_{1}$ & \multicolumn{2}{|c|}{$\tilde{\mathrm{A}}_{3}$} & $\tilde{A}_{5}$ & $\tilde{\mathrm{A}}_{5}$ & $\tilde{A}_{5}$ & $\tilde{\mathbf{D}}_{4}^{G=1}$ & $\tilde{\mathrm{D}}_{4}^{G=4}$ \\
\hline $\boldsymbol{\Gamma}_{o, p}$ & $\tilde{\mathrm{A}}_{1}$ & $\tilde{\mathrm{D}}_{4}^{O=1}$ & $\tilde{\mathrm{A}}_{3}$ & & $\tilde{\mathrm{A}}_{5}$ & $\tilde{\mathrm{D}}_{5}$ & $\tilde{\mathbf{E}}_{6}^{O=3}$ & $\tilde{\mathrm{D}}_{4}^{O=}$ & $\tilde{A}_{1}$ \\
\hline $\boldsymbol{\Gamma}_{p, g}$ & $\tilde{\mathrm{A}}_{1}$ & $\tilde{\mathrm{D}}_{4}^{P=4}$ & $\tilde{\mathrm{A}}_{3}$ & & $\tilde{\mathrm{D}}_{5}$ & $\tilde{\mathrm{A}}_{5}$ & $\tilde{\mathrm{E}}_{6}^{P=4}$ & $\tilde{\mathrm{A}}_{1}$ & $\tilde{\mathrm{D}}_{4}^{P=1}$ \\
\hline$(5-9) ?$ & $(5-6$ & $\mathbf{D}_{3}^{p}$ & cA & & no & no & $\mathbf{A}_{3}^{p}$ & $\mathbf{D}_{3}^{o}$ & $\mathbf{D}_{3}^{g}$ \\
\hline $\boldsymbol{\Gamma}_{g, o}$ & & $\tilde{\mathrm{D}}_{5}$ & $\tilde{\mathrm{D}}_{5}$ & $\tilde{D}_{5}$ & $\tilde{\mathrm{D}}_{5}$ & $\underbrace{\tilde{\mathbf{E}}_{6}^{G=}}_{6}$ & $\tilde{\mathbf{E}}_{6}^{G=3}$ & $\frac{\tilde{\mathbf{E}}_{6}^{G=4}}{\sim}$ & $\tilde{\mathbf{E}}_{6}^{G=4}$ \\
\hline$\overline{\boldsymbol{\Gamma}_{o, p}}$ & & $\tilde{\mathrm{A}}_{5}$ & $\tilde{\mathrm{D}}_{5}$ & $\tilde{\mathrm{D}}_{5}$ & $\tilde{\mathbf{E}}_{6}^{O=3}$ & $\tilde{\mathbf{E}}_{6}^{O=}=$ & $\tilde{\mathrm{E}}_{6}^{O=4}$ & $\tilde{\mathrm{A}}_{5}$ & $\tilde{\mathrm{D}}_{5}$ \\
\hline $\boldsymbol{\Gamma}_{p, g}$ & & $\tilde{D}_{5}$ & $\tilde{A}_{5}$ & $\tilde{\mathrm{D}}_{5}$ & $\tilde{\mathbf{E}}_{6}^{P=4}$ & $\tilde{A}_{5}$ & $\tilde{\mathrm{D}}_{5}$ & $\tilde{\mathbf{E}}_{6}^{P=3}$ & $\tilde{\mathbf{E}}_{6}^{P=3}$ \\
\hline$(5-9) ?$ & $?$ & no & no & no & no & $\mathbf{A}_{3}^{O}$ & no & $\mathbf{A}_{3}^{g}$ & no \\
\hline
\end{tabular}

$\left(\tilde{\mathrm{A}}_{2}\right.$ is ruled out since it does not allow a bicoloring.) The same holds for the other colors, of course.

One can now write down all potential candidates for the bicolored subgraphs:

Here we have indicated whether (5-9) is satisfied or not. One now checks that the remaining possibilities give solutions to $\mathrm{CP} 5.34$.

The solution (5-6) is not on the list of generalized ADE Dynkin diagrams, and we do not know whether this is an exception for $e=3$ or whether there exist more solutions which are not generalized ADE Dynkin diagrams for $e>3$. 
Example 5.39. For $e=0,1,2,3$ the list of transitive $\mathbb{N}_{[\mathrm{v}}^{0}$-representations given in (5-5) is almost complete. Adding a representation $\mathrm{M}_{\mathbf{c} \mathbf{A}_{3}}$ to this list, for any color by Lemma 5.32, and a representation for the special solution (5-6) completes the list, where one can check by hand that these are $\mathbb{N}_{[\mathrm{v}]}^{0}$-representations.

5B. Categorified story. Recall that $\left[\mathscr{T}_{e}\right]_{\oplus}^{\mathbb{C}} \cong \mathrm{T}_{e}$, see Proposition 4.48 (excluding $e=0$ ), and assume that we have a transitive 2-representation $\mathscr{M}$ of $\mathscr{T}_{e}$. Then $[\mathscr{M}]_{\oplus}^{\mathbb{C}}$ is a transitive $\mathbb{N}_{[\mathrm{v}]}^{0}$-representation of $\mathrm{T}_{e}$. So, by the discussion in Section $5 \mathrm{~A}$, the classification of simple transitive 2-representations of $\mathscr{T}_{e}$ boils down to CP 5.34 together with the construction of the corresponding 2-representations (i.e their categorification). We are going to explain this construction for types A and D.

Satake and 2-representations. Let us first sketch how Theorem 4.37 gives rise to a correspondence between the simple transitive 2-representations of $\mathscr{Q}_{e}^{G O P}$ and those of $\mathscr{T}_{e}$. We will discuss the details in the sections below.

Recall that there is a bijection between the equivalence classes of simple transitive 2-representations of $\mathscr{Q}_{e}^{G O P}$ and the Morita equivalence classes of simple algebra 1-morphisms in $\mathscr{Q}_{e}^{G O P}$, cf. [MMMT16, Theorem 9].

For $\mathscr{T}_{e}$ the situation is more complicated, because it is additive, but not abelian. However, it is still true that, if A is an indecomposable algebra 1-morphism in $\mathscr{T}_{e}$, then $\mathcal{M}_{\text {od }} \mathscr{T}_{e}(\mathrm{~A})$ is a transitive 2-representation of $\mathscr{T}_{e}$. By taking its simple quotient, as described in Remark 5.14, we get a simple transitive 2-representation associated to A.

As we will see, any algebra 1-morphism in $\mathrm{A}$ in $\mathcal{Q}_{e}$ gives rise to an algebra 1-morphism $\mathrm{A}^{\mathbf{u}}$ in $\mathscr{Q}_{e}^{G O P}(\mathbf{u}, \mathbf{u})$. (Without loss of generality, we will concentrate on the case $\mathbf{u}=g$ below.)

The Satake 2-functor from Lemma 4.47 transports $\mathrm{A}^{g}$ to an algebra 1-morphism in $\boldsymbol{m} \mathfrak{T}_{e}(g, g)$ (where we keep the same notation). Biinduction, which means gluing white outer regions to the diagrams which define the multiplication and unit 2-morphisms (see also Example 4.41), then gives an algebra 1-morphism $\mathrm{B}^{g}=\mathrm{B}\left(\mathrm{A}^{g}\right) \in \mathscr{T}_{e}=\boldsymbol{m} \mathscr{T}_{e}(\emptyset, \emptyset)$. As we will show, the algebra 1-morphism also has to be translated (which would correspond to shifting the grading if the algebra 1-morphism were given by a graded bimodule), so that the final degree of the unit and multiplication 2-morphisms becomes zero.

The fact that $\mathrm{B}^{g}$ is associative and unital, follows almost immediately from the associativity and unitality of $\mathrm{A}^{g}$. (For a detailed proof we refer to [MMMT16, Section 7.3].) By construction, $\mathrm{B}^{g}$ is indecomposable if $\mathrm{A}$ is simple.

Thus, given a simple transitive 2-representation $\mathscr{M}$ of $\mathscr{Q}_{e}^{G O P}$, let A be the corresponding simple algebra 1-morphism in $\mathscr{Q}_{e}^{G O P}$ and take $\mathscr{M}_{g}$ to be the simple quotient of $\mathcal{M o d}_{\mathscr{T}_{e}}\left(\mathrm{~B}^{g}\right)$.

Remark 5.40. Note that all simple algebra 1-morphisms in $\mathscr{Q}_{e}^{G O P}$ arise via coloring from simple algebra 1-morphisms in $\mathcal{Q}_{e}$. So our first task below is to recall the latter, which were already known, see e.g. [Sch17]. However, we present a self-contained construction in this paper. As a service to the reader, we also recall the proof of the known classification of their simple module 1-morphisms in $\mathcal{Q}_{e}$.

Type A 2-representations. The object $\mathrm{A}^{\mathbf{A}_{e}}=\mathrm{L}_{0,0} \cong \mathbb{C}$ is clearly a simple algebra object in $\mathcal{Q}_{e}$, because it is the identity object. Thus, coloring gives us a simple algebra 1-morphism $\mathrm{A}^{\mathbf{A}_{e}}$ in $\mathscr{Q}_{e}^{G O P}$. After applying the Satake 2-functor, we get $\mathbf{S}_{e}\left(\mathrm{~A}^{\mathbf{A}_{e}^{g}}\right)=g$, which is the identity 1-morphism in $\boldsymbol{m} \mathscr{T}_{e}(g, g)$.

Therefore, we have $\mathrm{B}^{\mathbf{A}_{e}^{g}}=\emptyset b g b \emptyset\{-3\} \in \mathscr{T}_{e}$, which is an indecomposable 1-morphism in $\mathscr{T}_{e}$. Recall that $\emptyset b g b \emptyset \cong \emptyset y g y \emptyset$, by Lemma 4.25. We have translated $\emptyset b g b \emptyset$ by -3 , so that the final degree of the unit and multiplication 2-morphisms below becomes zero. Note further that $\mathrm{B}^{\mathbf{A}_{e}}$ is a Frobenius 1-morphism, and its (co)unit and (co)multiplication 2-morphisms 
(with their respective unshifted degrees) are given by

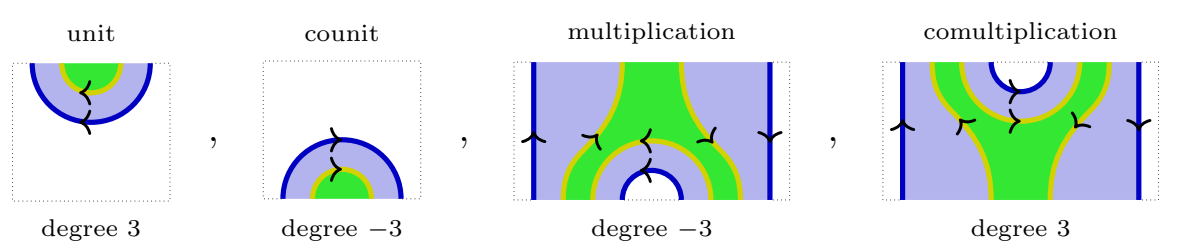

By construction, the corresponding simple transitive 2-representation $\mathscr{M}_{\mathbf{A}_{e}^{g}}$ of $\mathscr{T}_{e}$ is equivalent to a cell 2-representation and decategorifies to $\mathrm{M}_{\mathbf{A}_{e}^{g}}$ from Definition 5.27. Similarly for the secondary colors $o$ and $p$.

Type D 2-representations. Let $e \equiv 0 \bmod 3$. In this case, the decomposition of the algebra 1-morphism into simple 1-morphisms in $\mathcal{Q}_{e}$ is given by

$$
\mathrm{A}^{\mathbf{D}_{e}} \cong \mathrm{L}_{0,0} \oplus \mathrm{L}_{e, 0} \oplus \mathrm{L}_{0, e} .
$$

In order to define the multiplication and unit 2-morphisms of $\mathrm{A}^{\mathbf{D}_{e}}$ in $\mathcal{Q}_{e}$, recall from the representation theory of $\mathfrak{s l}_{3}$ that

$$
\left(\mathrm{L}_{e, 0}\right)^{*} \cong\left(\operatorname{Sym}^{e}\left(\mathbb{C}^{3}\right)\right)^{*} \cong \operatorname{Sym}^{e}\left(\left(\mathbb{C}^{3}\right)^{*}\right) \cong \mathrm{L}_{0, e},
$$

where ${ }^{*}$ means the dual module and $\operatorname{Sym}^{e}$ the $e^{\text {th }}$ symmetric power. Note that, by using e.g. [BZ08, Proposition 2.11], we have similar isomorphisms in the quantum case as well. Thus, in order to delineate the monoidal subcategory generated by the quantum symmetric powers, we can use the symmetric web categories described in [RT16], [TVW17], after adding the duals as in [QS15]. These symmetric web categories are built from certain labeled, trivalent graphs, and we need the monoidal subcategory of it generated by the objects $e, e^{*}$ and the morphisms

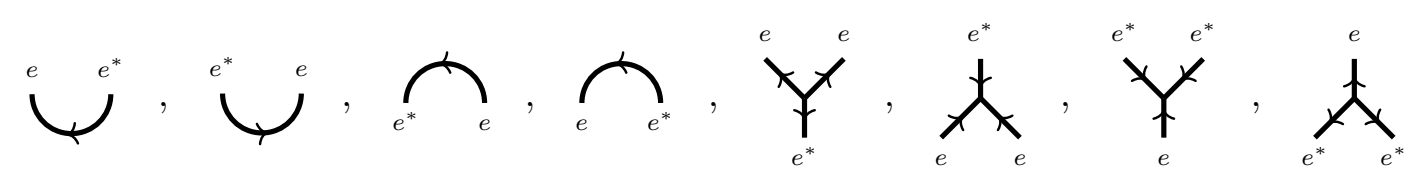

(our reading conventions are still from bottom to top), subject to some relations which are all stated e.g. in [RW18, Section 2.1]. These are basically thick, but uncolored versions of the webs which we met in Section $4 \mathrm{C}$, and the object $e$ corresponds to the $e^{\text {th }}$ quantum symmetric power of $\mathrm{L}_{1,0}$, which is $\mathrm{L}_{e, 0}$, and $e^{*}$ to its dual, which is $\mathrm{L}_{0, e}$.

Hence, we can use the diagrammatic calculus of symmetric webs to describe the intertwiners in $\mathcal{Q}_{\mathrm{q}}$ that we need. So far, we have assumed that $\mathrm{q}$ is a generic parameter. By putting it equal to a primitive, complex $2(e+3)^{\text {th }}$ root of unity $\eta$, we get a projection onto $\mathcal{Q}_{e}$, and we can use the specialized relations of the symmetric web calculus.

To be absolutely clear, we do not claim that the symmetric web calculi are equivalent to the monoidal subcategories in question. All we need is that the functor from the web calculus to $\mathcal{Q}_{e}$ is full, which is true.

We use the following shorthand:

$$
\mathrm{L}_{0,0} \leadsto \emptyset, \quad \mathrm{L}_{e, 0} \leadsto e, \quad \mathrm{~L}_{0, e} \leadsto e^{*},
$$

and $\mathrm{L}_{e, 0} \otimes \mathrm{L}_{0, e} \leadsto e e^{*}$ etc., where we omit the $\otimes$ symbol. 
Proposition 5.41. $\mathrm{A}^{\mathrm{D}_{e}}$ has the structure of a Frobenius object in $\mathcal{Q}_{e}$ with unit $\iota: \mathbb{C} \rightarrow \mathrm{D}^{e}$, $\iota(1)=\emptyset$, counit $\epsilon: \mathrm{D}^{e} \rightarrow \mathbb{C}, \epsilon(\emptyset)=1$ and multiplication $m: \mathrm{D}^{e} \otimes \mathrm{D}^{e} \rightarrow \mathrm{D}^{e}$ given by

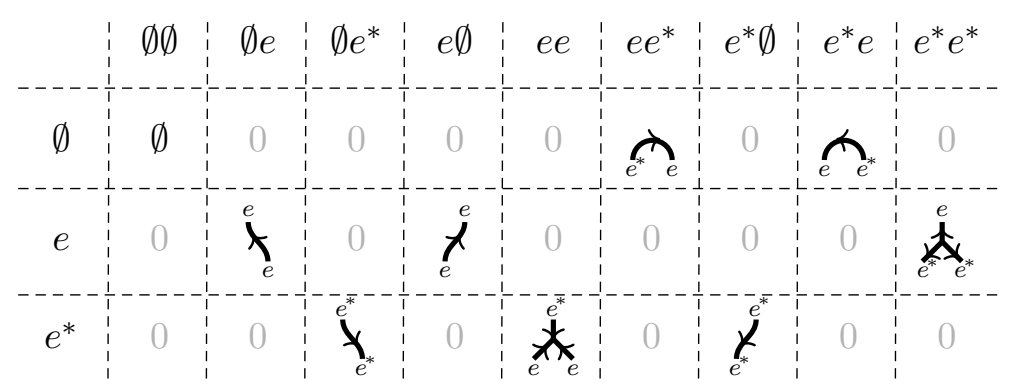

The comultiplication $\Delta: \mathrm{A}^{\mathbf{D}_{e}} \rightarrow \mathrm{A}^{\mathbf{D}_{e}} \otimes \mathrm{A}^{\mathbf{D}_{e}}$ is given by transposing (5-10) and turning the symmetric webs upside down.

We omit the edge labels (which are always $e$ or $e^{*}$ ) from now on.

Proof. First, we observe that the relations in the symmetric web calculus are invariant under horizontal and vertical reflections, which reduces the number of cases we need to verify. For example, checking the unitality of $\mathrm{A}^{\mathbf{D}_{e}}$ boils down to checking the commutativity of

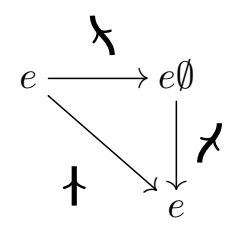

which follows directly from the symmetric web calculus.

Next, we show that $m$ and $\Delta$ are (co)associative. Up to symmetries and trivial compositions, we need to check that
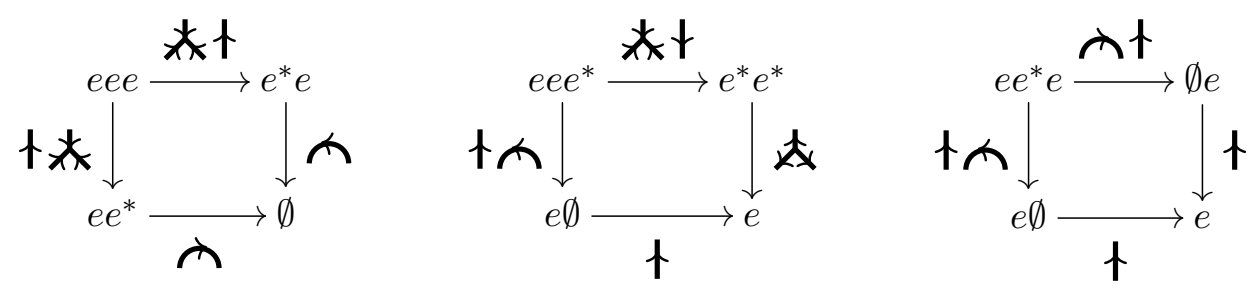

commute. The leftmost case is just an isotopy in the symmetric web calculus. The other two cases follow by observing that we have

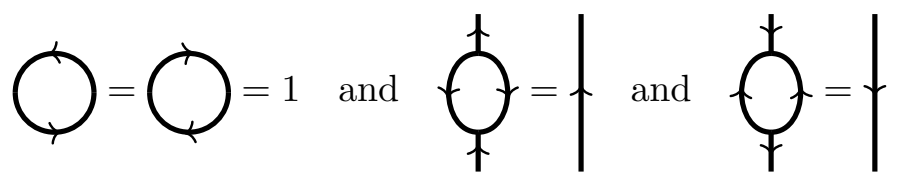

by specializing the relations in [RW18, Section 2.1]. Here it is crucial that $\eta$ is a $2(e+3)^{\text {th }}$ root of unity. For example, $[e+1]_{\eta}=[2]_{\eta}$ in this case, which implies that $\left[\begin{array}{c}e+2 \\ e\end{array}\right]_{\eta}=[e+1]_{\eta}[2]_{\eta}^{-1}=1$. The relations in (5-11) give
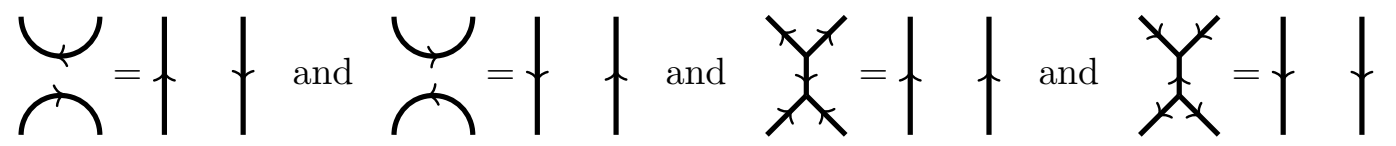

which are needed to show associativity. 
For $\mathrm{A}^{\mathrm{D}_{e}}$ to be Frobenius, we additionally need to check that
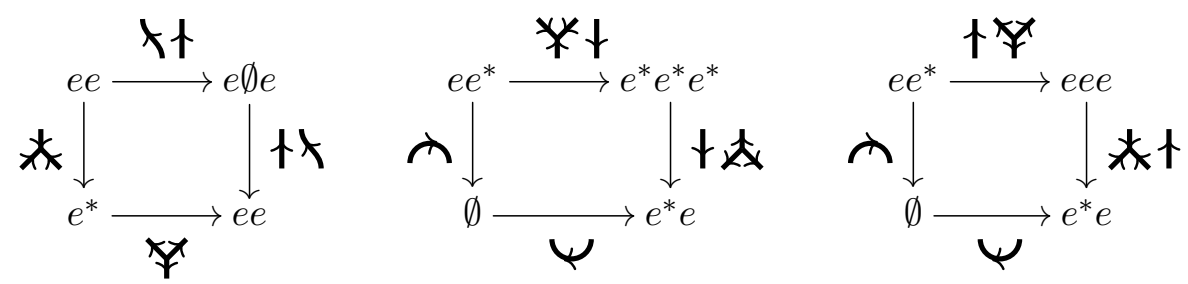

commute, which follows from (5-12). The other diagrammatic equations, which prove the compatibility between the multiplication and the comultiplication, are immediate.

Proposition 5.42. The rank of the module category associated to $\mathrm{A}^{\mathrm{D}_{e}}$ is equal to $\frac{t_{e}-1}{3}+3$, the number of nodes of the graph $\mathbf{D}_{e}$.

Note here that $t_{e}=\frac{(e+1)(e+2)}{2} \equiv 1 \bmod 3$ since $e \equiv 0 \bmod 3$, by assumption.

Proof. We first recall some facts. By [BK01, Section 3.3] or [Sch17, Lemma 3.2.1], the so-called twist of $\mathbf{A}^{\mathbf{D}_{e}}$ is the identity morphism. (This is false when $e \not \equiv 0 \bmod 3$.) Note also that $\mathrm{A}^{\mathbf{D}_{e}}$ is simple as an algebra 1-morphism. Moreover, let $\eta \operatorname{dim}(0)$ denote the quantum dimension of $0 \in \mathcal{Q}_{e}$, which is defined by specializing the generic quantum dimension at the primitive, complex root of unity $\eta$, see e.g. [EGNO15, Section 4.7]. By Weyl's character formula [Jan96, Theorem 5.15] and its specialization, we have

$$
\eta \operatorname{dim}\left(\mathrm{L}_{m, n}\right)=[2]_{\eta}^{-1}[m+1]_{\eta}[n+1]_{\eta}[m+n+2]_{\eta} .
$$

Hence, we get $\eta \operatorname{dim}\left(\mathrm{A}^{\mathbf{D}_{e}}\right)=3 \neq 0$. By [KJO02, Lemma 1.20], this implies that $\mathrm{A}^{\mathbf{D}_{e}}$ is rigid, as defined in [KJO02, Definition 1.11].

Therefore, $\mathcal{M o d}_{\mathcal{Q}_{e}}\left(\mathrm{~A}^{\mathbf{D}_{e}}\right)$ is a semisimple category with finitely many isomorphism classes of simples, by [KJO02, Theorem 3.3]. Furthermore, any simple module in $\mathcal{M o d}_{\mathcal{Q}_{e}}\left(\mathrm{~A}^{\mathbf{D}_{e}}\right)$ is a direct summand of $\mathrm{F}(0)$ for a certain $0 \in \mathcal{Q}_{e}$, by [KJO02, Lemma 3.4]. Here F : $\mathcal{Q}_{e} \rightarrow \mathcal{M}_{\mathcal{O}_{\mathcal{Q}_{e}}}\left(\mathrm{~A}^{\mathbf{D}_{e}}\right)$ is the free functor defined by $\mathrm{F}(\mathrm{O})=\mathbf{0} \otimes \mathrm{A}^{\mathbf{D}_{e}}$. By [KJO02, Lemma 1.16], this functor is biadjoint to the forgetful functor $\mathrm{G}: \mathcal{M}_{\mathcal{Q}_{e}}\left(\mathrm{~A}^{\mathbf{D}_{e}}\right) \rightarrow \mathcal{Q}_{e}$. As a last ingredient recall that

$$
\mathrm{L}_{m, n} \otimes \mathrm{L}_{e, 0} \cong \mathrm{L}_{e-m-n, m}, \quad \mathrm{~L}_{m, n} \otimes \mathrm{L}_{0, e} \cong \mathrm{L}_{n, e-m-n},
$$

hold in $\mathcal{Q}_{e}$ by e.g. [Saw06, Corollary 8].

It is now easy to determine the simples in $\operatorname{Mod}_{\mathcal{Q}_{e}}\left(\mathrm{~A}^{\mathbf{D}_{e}}\right)$. Let us write $e=3 r$.

Assume that $(m, n) \neq(r, r)$. Then

$$
\mathrm{GF}\left(\mathrm{L}_{m, n}\right) \cong \mathrm{L}_{m, n} \oplus \mathrm{L}_{e-m-n, m} \oplus \mathrm{L}_{n, e-m-n} .
$$

These three summands form a three element orbit of the cut-off of the positive Weyl chamber under the rotation by $\frac{2 \pi}{3}$. Therefore, we have

$$
\operatorname{dim}_{\mathbb{C}} \operatorname{Hom}_{\mathrm{A}^{\mathbf{D}_{e}}}\left(\mathrm{~F}\left(\mathrm{~L}_{m, n}\right), \mathrm{F}\left(\mathrm{L}_{m, n}\right)\right)=\operatorname{dim}_{\mathbb{C}} \operatorname{Hom}_{\mathcal{Q}_{e}}\left(\mathrm{~L}_{m, n}, \mathrm{GF}\left(\mathrm{L}_{m, n}\right)\right)=1 .
$$

By the categorical version of Schur's lemma, see e.g. [EGNO15, Lemma 1.5.2], F( $\left.\mathrm{L}_{m, n}\right)$

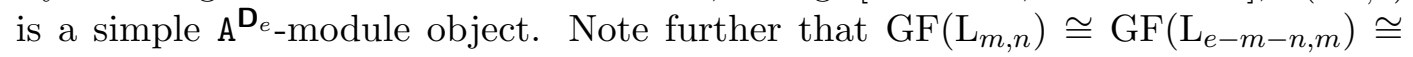
$\mathrm{GF}\left(\mathrm{L}_{n, e-m-n}\right)$. Thus, we have

$\operatorname{dim}_{\mathbb{C}} \operatorname{Hom}_{\mathrm{A} \mathbf{D}_{e}}\left(\mathrm{~F}\left(\mathrm{~L}_{m, n}\right), \mathrm{F}\left(\mathrm{L}_{e-m-n, m}\right)\right)=\operatorname{dim}_{\mathbb{C}} \operatorname{Hom}_{\mathrm{A}} \mathbf{D}_{e}\left(\mathrm{~F}\left(\mathrm{~L}_{m, n}\right), \mathrm{F}\left(\mathrm{L}_{n, e-m-n}\right)\right)=1$,

by adjointness of $\mathrm{F}$ and $\mathrm{G}$. Thus, $\mathrm{F}\left(\mathrm{L}_{m, n}\right) \cong \mathrm{F}\left(\mathrm{L}_{e-m-n, m}\right) \cong \mathrm{F}\left(\mathrm{L}_{n, e-m-n}\right)$.

Finally, $\mathrm{F}\left(\mathrm{L}_{m, n}\right) \neq \mathrm{F}\left(\mathrm{L}_{m^{\prime}, n^{\prime}}\right)$, if $\left(m^{\prime}, n^{\prime}\right) \notin\{(m, n),(e-m-n, m),(n, e-m-n)\}$, because in that case $\operatorname{GF}\left(\mathrm{L}_{m, n}\right) \nRightarrow \mathrm{GF}\left(\mathrm{L}_{m^{\prime}, n^{\prime}}\right)$. 
Assume that $(m, n)=(r, r)$. Then

$$
\mathrm{GF}\left(\mathrm{L}_{r, r}\right) \cong \mathrm{L}_{r, r} \oplus \mathrm{L}_{r, r} \oplus \mathrm{L}_{r, r} .
$$

Here $\mathrm{L}_{r, r}$ is the fixed point in the cut-off of the positive Weyl chamber under the rotation by $\frac{2 \pi}{3}$. Therefore, we have

$$
\operatorname{dim}_{\mathbb{C}} \operatorname{Hom}_{\mathrm{A}} \mathbf{D}_{e}\left(\mathrm{~F}\left(\mathrm{~L}_{r, r}\right), \mathrm{F}\left(\mathrm{L}_{r, r}\right)\right)=\operatorname{dim}_{\mathbb{C}} \operatorname{Hom}_{\mathcal{Q}_{e}}\left(\mathrm{~L}_{r, r}, \operatorname{GF}\left(\mathrm{L}_{r, r}\right)\right)=3 .
$$

This shows that $\mathrm{F}\left(\mathrm{L}_{r, r}\right)$ decomposes into three simples, each of which is mapped to $\mathrm{L}_{r, r}$ by $\mathrm{G}$, but which are pairwise non-isomorphic as $\mathrm{A}^{\mathbf{D}}$-module objects. (Otherwise, the dimension in (5-13) would be 5 or 9.)

The statement now follows by counting.

Example 5.43. In case $e=3$ (the case illustrated on the left in Figure App-4 below), we have ten simple objects in $\mathcal{Q}_{e}$ :

- $\mathrm{L}_{0,0}, \mathrm{~L}_{3,0}, \mathrm{~L}_{0,3}$, which have quantum dimension $[1]_{\eta}=1$.

- $\mathrm{L}_{1,0}, \mathrm{~L}_{2,1}, \mathrm{~L}_{0,2}$ and $\mathrm{L}_{0,1}, \mathrm{~L}_{1,2}, \mathrm{~L}_{2,0}$, which have quantum dimension $[3]_{\eta}=2$.

- $\mathrm{L}_{1,1}$, which has quantum dimension $[2]_{\eta}[4]_{\eta}=3$.

In contrast, the simple $\mathrm{A}^{\mathbf{D}_{3}}$-module objects are:

- $\mathrm{L}_{0,0} \oplus \mathrm{L}_{3,0} \oplus \mathrm{L}_{0,3}$, which have quantum dimension $3[1]_{\eta}=3$.

- $\mathrm{L}_{1,0} \oplus \mathrm{L}_{2,1} \oplus \mathrm{L}_{0,2}$ and $\mathrm{L}_{0,1} \oplus \mathrm{L}_{1,2} \oplus \mathrm{L}_{2,0}$, which have quantum dimension $3[3]_{\eta}=6$.

- Three non-isomorphic copies of $\mathrm{L}_{1,1}$, which still have quantum dimension 3 .

Remark 5.44. The above classification is consistent with the following analog of (3-20). Let

$$
\eta \operatorname{dim}\left(\mathcal{Q}_{e}\right)=\sum_{0 \leq m+n \leq e} \eta \operatorname{dim}\left(\mathrm{L}_{m, n}\right)^{2} .
$$

Since $\mathrm{A}^{\mathbf{D}_{e}}$ is rigid, we have

$$
\eta \operatorname{dim}\left(\mathrm{A}^{\mathbf{D}_{e}}\right) \eta \operatorname{dim}\left(\mathcal{Q}_{e}\right)=\sum_{\mathbf{S}} \eta \operatorname{dim}(\mathbf{S})^{2}
$$

where we sum over a complete set of pairwise non-isomorphic, simple $\mathrm{A}^{\mathbf{D}}{ }_{\text {-module objects }}$ S. The formula in (5-14) follows e.g. from [EGNO15, Example 7.16.9(ii)]. Note that in this example $\eta \operatorname{dim}(0)$ equals the Perron-Frobenius dimension of 0 as used in [EGNO15, Example 7.16.9(ii)] because $\mathrm{A}^{\mathbf{D}_{e}}$ is rigid.

By Proposition 5.41, we see that $\mathrm{A}^{\mathbf{D}_{e}}$ can be regarded as a Frobenius algebra 1-morphism in $\mathscr{Q}_{e}^{G O P}$. Hence, we get a Frobenius algebra 1-morphism $\mathrm{B}^{\mathbf{D}_{e}^{g}}\{-3\}$ in $\mathscr{T}_{e}$.

Remark 5.45. In this case it would be hard to write down explicitly the diagrams which define the structural 2-morphisms of $\mathrm{B}^{\mathbf{D}_{e}^{g}}$, i.e. unit, multiplication, counit and comultiplication. The reason is that the symmetric web calculus suppresses the clasps corresponding to $\mathrm{L}_{e, 0}$ and $\mathrm{L}_{0, e}$, i.e. the quantum symmetrizers and antisymmetrizers on $e$ strands in Kuperberg's web calculus [Kup96], cf. Example 4.41.

By Proposition 5.42, $\mathscr{M}_{\mathbf{D}_{e}^{g}}$ does not correspond to the cell 2-representation if $e \equiv 0 \bmod 3$, and, by construction, it categorifies $\mathbf{M}_{\mathbf{D}_{e}^{g}}$ from Definition 5.27. Similarly for $o$ and $p$.

Some simple transitive 2-representations. Note that an equivalence of simple transitive 2-representations decategorifies to a $\mathbb{N}_{[\mathrm{v}]}^{0}$-equivalence of transitive $\mathbb{N}_{[\mathrm{v}]}^{0}$-representations. Hence, the following is the summary of the above and Lemma 5.32: 
Theorem 5.46. Let us indicate by $g, o, p$ the starting color. Then we have (at least) the following simple transitive 2-representations of $\mathscr{T}_{e}$.

\begin{tabular}{r|c:c} 
& $e \equiv 0 \bmod 3$ & $e \not \equiv 0 \bmod 3$ \\
\hdashline 2-reps. & $\mathscr{M}_{\mathbf{A}_{e}^{g}}, \mathscr{M}_{\mathbf{A}_{e}^{o}}, \mathscr{M}_{\mathbf{A}_{e}^{p}}$, & $\mathscr{M}_{\mathbf{A}_{e}^{g}}$ \\
& $\mathscr{M}_{\mathbf{D}_{e}^{g}}, \mathscr{M}_{\mathbf{D}_{e}^{o}}, \mathscr{M}_{\mathbf{D}_{e}^{p}}$ & \\
quantity & 6 & 1
\end{tabular}

Moreover, the 2-representations $\mathscr{M}_{\mathbf{A}_{e}}$ are the cell 2-representations of $\mathscr{T}_{e}$, and all of these decategorify to the corresponding $\mathrm{T}_{e}$-representations in (5-5).

We note here that the case $e=0$ is not included in our discussion above, but Theorem 5.46 holds in this case as well (but types A and D coincide), which can be checked directly.

5C. Trihedral zig-zag algebras. We are going to describe a weak categorification of the $\mathbb{N}_{[\mathrm{v}]}^{0}$-representations $\mathrm{M}_{\mathbf{A}_{\infty}}$ and $\mathrm{M}_{\mathbf{A}_{e}}$ from Section $5 \mathrm{~A}$ using a certain quiver algebra. (By weak categorification we mean the same as e.g. in [KMS09, Definition 1].)

Below we let $i, j, k$ always be different elements in $\{x, y, z\}$. Moreover, we write $\dot{i}_{m, n}$ for the idempotent at a given vertex labeled by $(m, n)$, and a path from $\mathbf{i}_{m, n}$ to $\boldsymbol{j}_{m^{\prime}, n^{\prime}}$ is denoted by $j\left|i=j_{m^{\prime}, n^{\prime}} \circ j\right| i \circ i_{m, n}$ (abusing notation, we omit the idempotents) with compositions $k|j \circ j| i=k|j| i$ and $j\left|i \circ \alpha_{k}=j\right| i \alpha_{k}$ etc.

The trihedral zig-zag algebra of level $\infty$. We work over $\mathbb{C}$ in this section.

Definition 5.47. Let $\nabla_{*}$ be the path algebra of the following quiver.

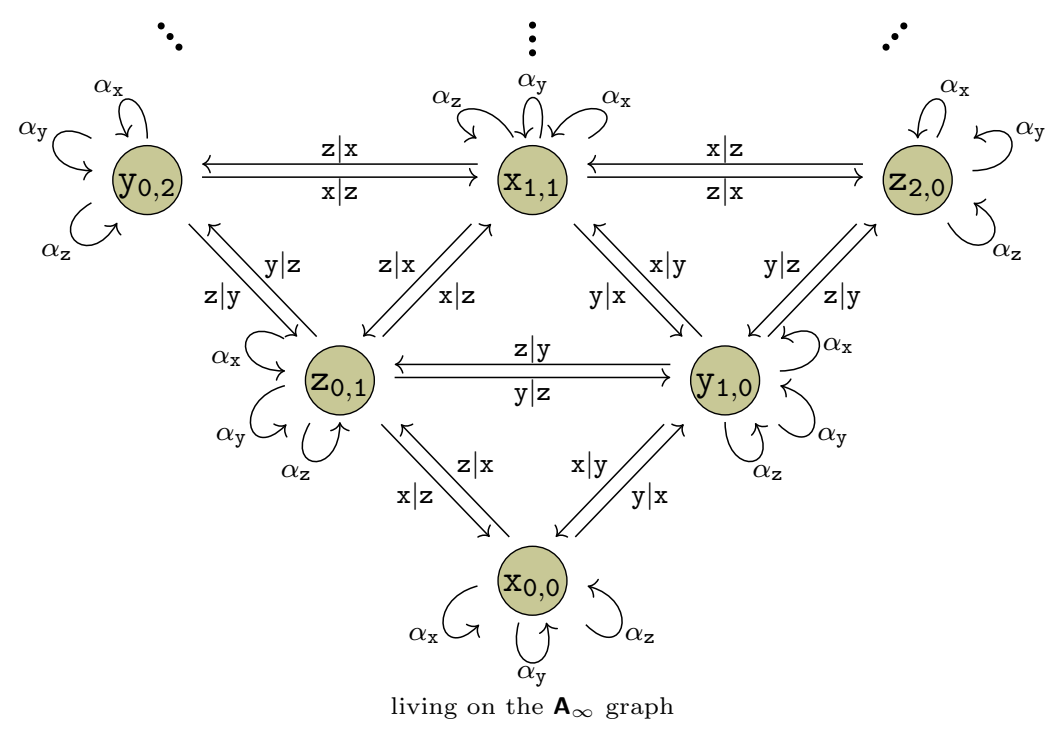

We view $\nabla_{*}$ as being graded by putting paths $\mathrm{j} \mid \mathrm{i}$ and loops $\alpha_{\mathrm{i}}$ in degree 2 .

Definition 5.48. Let $\nabla_{\infty}$ be the quotient algebra of $\nabla_{*}$ by the following relations.

(5.48.a) Leaving a triangular face is zero. Any oriented path of length two between non-adjacent vertices is zero.

(5.48.b) The relations of the cohomology ring of the variety of full flags in $\mathbb{C}^{3}$ : $\alpha_{\mathrm{i}} \alpha_{\mathrm{j}}=\alpha_{\mathrm{j}} \alpha_{\mathrm{i}}, \alpha_{\mathrm{x}}+\alpha_{\mathrm{y}}+\alpha_{\mathrm{z}}=0, \alpha_{\mathrm{x}} \alpha_{\mathrm{y}}+\alpha_{\mathrm{x}} \alpha_{\mathrm{z}}+\alpha_{\mathrm{y}} \alpha_{\mathrm{z}}=0$ and $\alpha_{\mathrm{x}} \alpha_{\mathrm{y}} \alpha_{\mathrm{z}}=0$.

(5.48.c) Sliding loops: $\mathrm{j}\left|\mathrm{i} \alpha_{\mathrm{i}}=-\alpha_{\mathrm{j}} \mathrm{j}\right| \mathrm{i}, \mathrm{j}\left|\mathrm{i} \alpha_{\mathrm{j}}=-\alpha_{\mathrm{i}} \mathrm{j}\right| \mathrm{i}$ and $\mathrm{j}\left|\mathrm{i} \alpha_{\mathrm{k}}=\alpha_{\mathrm{k}} \mathrm{j}\right| \mathrm{i}=0$.

(5.48.d) Zig-zag: $i|j| i=\alpha_{i} \alpha_{j}$. 
(5.48.e) Zig-zig equals zag times loop: $k|j| i=k\left|i \alpha_{i}=-\alpha_{k} k\right| i$.

We call $\nabla_{\infty}$ the trihedral zig-zag algebra of level $\infty$.

The relations (5.48.a) to (5.48.e) are homogeneous with respect to the degree defined in Definition 5.47, which endows $\nabla_{\infty}$ with the structure of a graded algebra, and we write $\operatorname{vdim}\left(\_\right) \in \mathbb{N}^{0}[\mathrm{v}]$ for the graded dimension, viewing $\mathrm{v}$ as a variable of degree 1 . Note that $\nabla_{\infty}$ is zero in all odd degrees, by definition.

Some basic properties.

Proposition 5.49. $\nabla_{\infty}$ is quadratic, i.e. it is generated in degree 2 and the relations are generated in degree 4 .

Proof. All relations except $\alpha_{\mathrm{x}}+\alpha_{\mathrm{y}}+\alpha_{\mathrm{z}}=0$ and $\alpha_{\mathrm{x}} \alpha_{\mathrm{y}} \alpha_{\mathrm{z}}=0$ are of degree 4 .

The degree two relation shows that our presentation is redundant: we could give a presentation with fewer generators and no degree two relation. We prefer our presentation, which is more symmetric and therefore easier to write down. But one could get rid of the degree two relation by using only two degree two loops per vertex.

Thus, up to base change, remains to show that $\alpha_{\mathrm{x}} \alpha_{\mathrm{y}} \alpha_{\mathrm{z}}=0$ is a consequence of degree 4 relations, which can be done as follows:

$$
\alpha_{\mathrm{x}} \alpha_{\mathrm{y}} \alpha_{\mathrm{z}} \stackrel{(5.48 . d)}{=} \mathrm{x}|\mathrm{y}| \mathrm{x} \alpha_{\mathrm{z}} \stackrel{(5.48 . c)}{=} 0 .
$$

This finishes the proof that $\nabla_{\infty}$ is quadratic.

Lemma 5.50. Let $\mathrm{S}=\{(m \pm 1, n),(m \pm 1, n \mp 1),(m, n \pm 1)\}$.

$$
\begin{aligned}
& \operatorname{Hom}_{\mathbb{C}}\left(\dot{i}_{m, n}, \dot{\mathbf{i}}_{m^{\prime}, n^{\prime}}\right)= \begin{cases}\mathbb{C}\left\{\mathbf{i}_{m, n}, \alpha_{\dot{i}}, \alpha_{j}, \alpha_{\mathrm{i}} \alpha_{\mathrm{j}}, \alpha_{\mathrm{i}} \alpha_{\mathrm{k}}, \alpha_{\mathbf{i}}^{2} \alpha_{j}\right\}, & \text { if }(m, n)=\left(m^{\prime}, n^{\prime}\right), \\
0, & \text { else, }\end{cases} \\
& \operatorname{Hom}_{\mathbb{C}}\left(\mathbf{i}_{m, n}, j_{m^{\prime}, n^{\prime}}\right)= \begin{cases}\mathbb{C}\left\{j|\mathbf{i}, j| \mathbf{i} \alpha_{\mathbf{i}}\right\}, & \text { if }\left(m^{\prime}, n^{\prime}\right) \in \mathrm{S}, \\
0, & \text { else. }\end{cases}
\end{aligned}
$$

Moreover, the non-trivial graded dimensions are $\operatorname{vdim}\left(\operatorname{Hom}_{\mathbb{C}}\left(\dot{i}_{m, n}, \dot{i}_{m, n}\right)\right)=\mathrm{v}^{3}[3]_{\mathrm{v}}$ ! and $\operatorname{vdim}\left(\operatorname{Hom}_{\mathbb{C}}\left(\mathbf{i}_{m, n}, \mathbf{j}_{m^{\prime}, n^{\prime}}\right)\right)=\mathrm{v}[2]_{\mathrm{v}}$, when $\left(m^{\prime}, n^{\prime}\right) \in \mathrm{S}$.

Proof. This is clear for the trivial hom-spaces by (5.48.a). So let us focus on the non-trivial ones. To this end, we first consider homogeneous linear combinations of loops of degree 2, 4, 6:

$$
\begin{gathered}
\alpha_{\mathrm{x}}+\alpha_{\mathrm{y}}=-\alpha_{\mathrm{z}}, \\
\alpha_{\mathrm{x}}^{2}+\alpha_{\mathrm{x}} \alpha_{\mathrm{y}}=-\alpha_{\mathrm{x}} \alpha_{\mathrm{z}}, \quad \alpha_{\mathrm{x}} \alpha_{\mathrm{y}}+\alpha_{\mathrm{y}}^{2}=-\alpha_{\mathrm{y}} \alpha_{\mathrm{z}}, \quad \alpha_{\mathrm{x}} \alpha_{\mathrm{z}}+\alpha_{\mathrm{y}} \alpha_{\mathrm{z}}=-\alpha_{\mathrm{z}}^{2}, \\
\alpha_{\mathrm{x}}^{2} \alpha_{\mathrm{z}}=\alpha_{\mathrm{x}} \alpha_{\mathrm{y}}^{2}=\alpha_{\mathrm{y}} \alpha_{\mathrm{z}}^{2}=-\alpha_{\mathrm{x}}^{2} \alpha_{\mathrm{y}}=-\alpha_{\mathrm{y}}^{2} \alpha_{\mathrm{z}}=-\alpha_{\mathrm{x}} \alpha_{\mathrm{z}}^{2} .
\end{gathered}
$$

These relations follow immediately from (5.48.a), and show that the endomorphism space of any vertex is spanned by the ones in (5-16).

Next, we consider all homogeneous elements of degree 4 in $\nabla_{*}$. The ones that are composites of two paths leaving a triangular face are zero by (5.48.a), and the remaining ones are linear combinations of those appearing in (5.48.c), (5.48.d), (5.48.e).

The homogeneous elements of degree 6 that are annihilated by (5.48.a) are

$$
\begin{aligned}
& \mathrm{i}|\mathrm{k}| \mathrm{j}|\mathrm{i} \stackrel{(5.48 . e)}{=} \mathrm{i}| \mathrm{k}\left|\mathbf{i} \alpha_{\mathrm{i}} \stackrel{(5.48 . d)}{=} \alpha_{\mathbf{i}}^{2} \alpha_{\mathrm{k}} \stackrel{(5-17)}{=}-\alpha_{\mathrm{i}}^{2} \alpha_{\mathrm{j}} \stackrel{(5.48 . d)}{=}-\mathrm{i}\right| \mathrm{j}\left|\mathbf{i} \alpha_{\mathrm{i}} \stackrel{(5.48 . e)}{=}-\mathrm{i}\right| \mathrm{j}|\mathrm{k}| \mathrm{i}, \\
& \mathrm{j}|\mathrm{i}| \mathrm{j}|\mathbf{i} \stackrel{(5.48 . d)}{=} \mathrm{j}| \mathbf{i} \alpha_{\mathrm{i}} \alpha_{\mathrm{j}} \stackrel{(5.48 . b)}{=} \mathrm{j} \mid \mathrm{i}\left(-\alpha_{\mathrm{i}} \alpha_{\mathrm{k}}-\alpha_{\mathrm{j}} \alpha_{\mathrm{k}}\right) \stackrel{(5.48 . c)}{=} 0 \text {, } \\
& \mathrm{k}|\mathrm{i}| \mathrm{j}|\mathrm{i} \stackrel{(5.48 . d)}{=} \mathrm{k}| \mathrm{i} \alpha_{\mathrm{i}} \alpha_{\mathrm{j}} \stackrel{(5.48 . c)}{=} 0
\end{aligned}
$$

including versions obtained by changing sources and targets. 
Finally, we claim that all homogeneous elements of degree $>6$ in $\nabla_{*}$ are zero in $\nabla_{\infty}$. For paths leaving a face or composites of only loops, there is nothing to show by (5.48.a) and (5.48.b). For paths of length four around one triangular face, we get

$$
\begin{aligned}
& \mathrm{j}|\mathrm{i}| \mathrm{k}|\mathrm{j}| \mathrm{i} \stackrel{(5.48 . e)}{=} \mathrm{j}|\mathrm{i}| \mathrm{k}\left|\mathrm{i} \alpha_{\mathrm{i}} \stackrel{(5.48 . d)}{=} \mathrm{j}\right| \mathrm{i} \alpha_{\mathrm{i}}^{2} \alpha_{\mathrm{k}} \stackrel{(5.48 . c)}{=} 0, \\
& \mathrm{k}|\mathrm{i}| \mathrm{k}|\mathrm{j}| \mathrm{i} \stackrel{(5.48 . e)}{=} \mathrm{k}|\mathrm{i}| \mathrm{k}\left|\mathrm{i} \alpha_{\mathrm{i}} \stackrel{(5.48 . d)}{=} \mathrm{k}\right| \mathrm{i} \alpha_{\mathrm{i}}^{2} \alpha_{\mathrm{k}} \stackrel{(5.48 . b)}{=}-\mathrm{k} \mid \mathrm{i} \alpha_{\mathrm{i}}^{2} \alpha_{\mathrm{j}} \stackrel{(5.48 . c)}{=} 0 \text {, } \\
& \mathrm{i}|\mathbf{j}| \mathbf{i}|\mathbf{j}| \mathbf{i} \stackrel{(5.48 . d)}{=} \mathbf{i}|\mathbf{j}| \mathbf{i} \alpha_{\mathbf{i}} \alpha_{\mathbf{j}} \stackrel{(5.48 . d)}{=} \alpha_{\mathbf{i}}^{2} \alpha_{\mathbf{j}}^{2} \stackrel{(5.48 . c)}{=} 0 \text {, } \\
& \mathrm{i}|\mathrm{k}| \mathrm{i}|\mathrm{j}| \mathrm{i} \stackrel{(5.48 . d)}{=} \mathrm{i}|\mathrm{k}| \mathrm{i} \alpha_{\mathrm{i}} \alpha_{\mathrm{j}} \stackrel{(5.48 . d)}{=} \alpha_{\mathrm{i}}^{2} \alpha_{\mathrm{j}} \alpha_{\mathrm{k}} \stackrel{(5.48 . c)}{=} 0 \text {. }
\end{aligned}
$$

Again, there are analogous relations obtained by changing sources and targets. Altogether this shows that the sets in (5-16) span the hom-spaces.

To show linear independence, we consider the following linear map, which on monomials in the path algebra is given by:

$$
\operatorname{tr}: \nabla_{\infty} \rightarrow \mathbb{C}, \quad \operatorname{tr}(a)= \begin{cases}1, & \text { if } a \in\left\{\alpha_{\mathrm{x}}^{2} \alpha_{\mathrm{z}}, \alpha_{\mathrm{x}} \alpha_{\mathrm{y}}^{2}, \alpha_{\mathrm{y}} \alpha_{\mathrm{z}}^{2}\right\}, \\ -1, & \text { if } a \in\left\{\alpha_{\mathrm{x}}^{2} \alpha_{\mathrm{y}}, \alpha_{\mathrm{y}}^{2} \alpha_{\mathrm{z}}, \alpha_{\mathrm{x}} \alpha_{\mathrm{z}}^{2}\right\}, \\ 0, & \text { else. }\end{cases}
$$

Note that tr is well-defined, which can be checked by showing that the relations are preserved. It is also easy to see that tr is a non-degenerate and symmetric trace form, e.g.

$$
\begin{gathered}
\operatorname{tr}(\mathrm{y}|\mathrm{x} \cdot \mathrm{x}| \mathrm{z} \mid \mathrm{y})=\operatorname{tr}(\mathrm{x}|\mathrm{z}| \mathrm{y} \mid \mathrm{x}) \stackrel{(5.48 . e)}{=} \operatorname{tr}\left(\mathrm{x}|\mathrm{y}| \mathrm{x} \alpha_{\mathrm{x}}\right) \stackrel{(5.48 . d)}{=} \operatorname{tr}\left(\alpha_{\mathrm{x}}^{2} \alpha_{\mathrm{y}}\right) \\
\stackrel{(5-17)}{=} \operatorname{tr}\left(-\alpha_{\mathrm{x}} \alpha_{\mathrm{y}}^{2}\right) \stackrel{(5.48 . d)}{=}-\operatorname{tr}\left(\alpha_{\mathrm{y}} \mathrm{y}|\mathrm{x}| \mathrm{y}\right) \stackrel{(5.48 . e)}{=} \operatorname{tr}(\mathrm{y}|\mathrm{x}| \mathrm{z} \mid \mathrm{y})=\operatorname{tr}(\mathrm{x}|\mathrm{z}| \mathrm{y} \cdot \mathrm{y} \mid \mathrm{x})
\end{gathered}
$$

We can now write down sets of morphisms which are dual to the ones from (5-16) with respect to tr, e.g.:

$$
\begin{aligned}
& \left\{\mathbf{i}_{m, n}, \alpha_{\mathrm{x}}, \alpha_{\mathrm{y}}, \alpha_{\mathrm{x}} \alpha_{\mathrm{y}}, \alpha_{\mathrm{x}} \alpha_{\mathrm{z}}, \alpha_{\mathrm{x}}^{2} \alpha_{\mathrm{z}}\right\} \leadsto\left\{\alpha_{\mathrm{x}}^{2} \alpha_{\mathbf{z}}, \alpha_{\mathrm{x}} \alpha_{\mathrm{z}}, \alpha_{\mathrm{x}} \alpha_{\mathrm{y}}, \alpha_{\mathrm{y}}, \alpha_{\mathrm{x}}, \mathbf{i}_{m, n}\right\}, \\
& \left\{j|\mathbf{i}, \mathbf{j}| \mathbf{i} \alpha_{\mathbf{i}}\right\} \leadsto\left\{ \pm \mathbf{i}\left|\mathbf{j} \alpha_{j}, \pm \mathbf{i}\right| \mathbf{j}\right\} \text {. }
\end{aligned}
$$

Since these sets span the corresponding hom-spaces, we are done.

The following result follows immediately from the proof of Lemma 5.50.

Corollary 5.51. $\nabla_{\infty}$ is a positively graded, symmetric Frobenius algebra.

The quotient of level $e$.

Definition 5.52. For fixed level $e$, let $\mathrm{K}_{e}$ be the two-sided ideal in $\nabla_{\infty}$ generated by

$$
\left\{\dot{i}_{m, n} \mid m+n \geq e+1\right\} .
$$

We define the trihedral zig-zag algebra of level $e$ as

$$
\nabla_{e}=\nabla_{\infty} / \mathrm{K}_{e}
$$

and we call $\mathrm{K}_{e}$ the vanishing zig-zag ideal of level $e$.

Clearly, $\nabla_{e}$ has a basis given by the elements in (5-16) for $m+n \leq e$. Thus, $\nabla_{e}$ is a finite-dimensional, positively graded algebra, which is a symmetric Frobenius algebra by Corollary 5.51. By the proof of Proposition 5.49 it is also quadratic, as long as $e \neq 0$. 
Weak categorification. Following ideas from [KS02], [AT17] and [MT16, Section 2], we let $\mathrm{P}_{\mathbf{i}_{m, n}}$, respectively $\dot{i}_{m, n} \mathrm{P}$, denote the left, respectively right, ideals in $\nabla_{\infty}$ generated by $\dot{i}_{m, n}$. These are indecomposable, graded projective $\nabla_{\infty}$-modules, and all indecomposable, graded projective left, respectively right, $\nabla_{\infty}$-modules are of this form, up to grading shifts.

By the above, $\mathrm{P}_{\mathbf{i}_{m, n}} \otimes \nabla_{\infty} \mathbf{i}_{m, n} \mathrm{P}$ is a biprojective $\nabla_{\infty}$-bimodule, i.e. it is projective as a left and as a right $\nabla_{\infty}$-module. Therefore,

$$
\begin{gathered}
\boldsymbol{\Theta}_{g}\left({ }_{-}\right)=\oplus_{\mathbf{x}_{m, n}}\left(\mathrm{P}_{\mathbf{x}_{m, n}} \otimes_{\mathbf{x}_{m, n}} \mathrm{P}\{-3\}\right) \otimes \nabla_{\infty}, \quad \boldsymbol{\Theta}_{o}\left(\_\right)=\oplus_{\mathbf{y}_{m, n}}\left(\mathrm{P}_{\mathbf{y}_{m, n}} \otimes_{\mathbf{y}_{m, n}} \mathrm{P}\{-3\}\right) \otimes \nabla_{\infty}, \\
\boldsymbol{\Theta}_{p}\left(\_\right)=\bigoplus_{z_{m, n}}\left(\mathrm{P}_{\mathbf{z}_{m, n}} \otimes_{\mathbf{z}_{m, n}} \mathrm{P}\{-3\}\right) \otimes \nabla_{\infty},
\end{gathered}
$$

define endofunctors on the category $\nabla_{e}$-p $\mathcal{M o d}^{\mathrm{gr}}$ of finite-dimensional, graded projective (left) $\nabla_{e}$-modules. Here $\otimes$ denotes $\otimes_{\mathbb{C}}$.

To state the weak categorification we denote by $\mathscr{E}$ nd $\left(\nabla_{e}-\right.$ p $\mathcal{M}$ od $\left.^{\text {gr }}\right)$ the category of endofunctors on $\nabla_{e}$-pMod ${ }^{\text {gr }}$. Considering $\mathrm{T}_{\infty}$ as a one-object category with the formal object $*$, we get the following.

Lemma 5.53. The functor $\mathrm{T}_{\infty} \rightarrow \mathscr{E} \mathrm{nd}\left(\nabla_{e}-\mathrm{p}_{\mathcal{M}} \mathrm{od}^{\mathrm{gr}}\right)$ given by $* \mapsto \nabla_{e}$-pMod $^{\mathrm{gr}}$ and

$$
\theta_{g} \mapsto \boldsymbol{\Theta}_{g}\left({ }_{-}\right), \quad \theta_{o} \mapsto \boldsymbol{\Theta}_{o}\left({ }_{-}\right), \quad \theta_{p} \mapsto \boldsymbol{\Theta}_{p}\left({ }_{-}\right)
$$

is well-defined.

Moreover, decategorification gives the transitive $\mathbb{N}_{[\mathrm{v}]}^{0}$-representation $\mathrm{M}_{\mathbf{A}_{\infty}}$ of $\mathrm{T}_{\infty}$.

Proof. Let us first show that $\boldsymbol{\Theta}_{g} \boldsymbol{\Theta}_{g} \cong \boldsymbol{\Theta}_{g}^{\oplus[3]_{\mathrm{v}} !}$, with the superscript $\oplus[3]_{\mathrm{v}}$ ! meaning six degree-shifted copies of $\boldsymbol{\Theta}_{g}$, with v corresponding to a degree-shift by one. Similar arguments show that the same holds for $\boldsymbol{\Theta}_{o}$ and $\boldsymbol{\Theta}_{p}$, of course. Note that $\boldsymbol{\Theta}_{g} \boldsymbol{\Theta}_{g}$ is given by tensoring with the bimodule

$$
\oplus_{\mathbf{x}_{m, n}} \mathrm{P}_{\mathrm{x}_{m, n}} \otimes \mathrm{x}_{m, n} \mathrm{P} \otimes \nabla_{\infty} \mathrm{P}_{\mathrm{x}_{m, n}} \otimes \mathrm{x}_{m, n} \mathrm{P}\{-6\}
$$

with all other direct summands being zero. By definition, this is isomorphic to

$$
\bigoplus_{\mathbf{x}_{m, n}} \mathrm{P}_{\mathbf{x}_{m, n}} \otimes \operatorname{End}_{\nabla_{\infty}}\left(\mathrm{x}_{m, n}\right) \otimes \mathrm{x}_{m, n} \mathrm{P}\{-6\} \cong \oplus_{\mathbf{x}_{m, n}}\left(\mathrm{P}_{\mathbf{x}_{m, n}} \otimes \mathrm{x}_{m, n} \mathrm{P}\{-3\}\right)^{\oplus[3]_{\mathrm{v}} !},
$$

where the displayed isomorphism follows from Lemma 5.50.

Next, we show that $\boldsymbol{\Theta}_{g} \boldsymbol{\Theta}_{o} \boldsymbol{\Theta}_{g} \cong \boldsymbol{\Theta}_{g} \boldsymbol{\Theta}_{p} \boldsymbol{\Theta}_{g}$. Again, similar arguments show the analogous result in the remaining cases. The functor $\boldsymbol{\Theta}_{g} \boldsymbol{\Theta}_{o} \boldsymbol{\Theta}_{g}$ is given by tensoring with

$$
\oplus \mathrm{P}_{\mathrm{x}_{m, n}} \otimes \mathrm{x}_{m, n} \mathrm{P} \otimes \nabla_{\infty} \mathrm{P}_{\mathrm{y}_{m^{\prime}, n^{\prime}}} \otimes_{\mathrm{y}_{m^{\prime}, n^{\prime}}} \mathrm{P} \otimes \nabla_{\infty} \mathrm{P}_{\mathrm{x}_{m^{\prime \prime}, n^{\prime \prime}}} \otimes_{\mathrm{x}_{m^{\prime \prime}, n^{\prime \prime}}} \mathrm{P}\{-9\},
$$

where the direct sum is over all neighboring pairs $(m, n),\left(m^{\prime}, n^{\prime}\right)$ and $\left(m^{\prime}, n^{\prime}\right),\left(m^{\prime \prime}, n^{\prime \prime}\right)$, i.e. $\left(m^{\prime}, n^{\prime}\right) \in\{(m \pm 1, n),(m, n \pm 1),(m \pm 1, n \mp 1)\}$ and $\left(m^{\prime \prime}, n^{\prime \prime}\right) \in\{(m, n),(m \pm 1, n \pm 1),(m \pm$ $2, n \mp 1),(m \pm 1, n \mp 2)\}$. This is isomorphic to

$$
\begin{gathered}
\oplus \mathrm{P}_{\mathrm{x}_{m, n}} \otimes \operatorname{Hom}_{\nabla_{\infty}}\left(\mathrm{x}_{m, n}, \mathrm{y}_{m^{\prime}, n^{\prime}}\right) \otimes \operatorname{Hom}_{\nabla_{\infty}}\left(\mathrm{y}_{m^{\prime}, n^{\prime}}, \mathrm{x}_{m^{\prime \prime}, n^{\prime \prime}}\right) \otimes{\mathrm{x}_{m^{\prime \prime}, n^{\prime \prime}}} \mathrm{P}\{-9\} \cong \\
\oplus\left(\mathrm{P}_{\mathbf{x}_{m, n}} \otimes{\mathrm{x}_{m^{\prime \prime}, n^{\prime \prime}}} \mathrm{P}\{-7\}\right)^{\oplus[2]_{\mathrm{v}}^{2}},
\end{gathered}
$$

where the superscript $\oplus[2]_{\mathrm{v}}^{2}$ should be interpreted as before. The isomorphism displayed in (5-19) follows from Lemma 5.50.

The functor $\boldsymbol{\Theta}_{g} \boldsymbol{\Theta}_{O} \boldsymbol{\Theta}_{g}$ is given by tensoring with the $\nabla_{\infty}$-bimodule obtained from the one in (5-18) by replacing $\mathrm{y}_{m^{\prime}, n^{\prime}}$ with $\mathbf{z}_{m^{\prime}, n^{\prime}}$, which is also isomorphic to the $\nabla_{\infty}$-bimodule in (5-19). Although the neighboring pairs change when we replace $\mathbf{y}_{m^{\prime}, n^{\prime}}$ with $\mathbf{z}_{m^{\prime}, n^{\prime}}$, their total number is equal by symmetry. Thus, the final number of direct summands in (5-19) is the same in both cases. This finishes the proof that $\boldsymbol{\Theta}_{g} \boldsymbol{\Theta}_{o} \boldsymbol{\Theta}_{g} \cong \boldsymbol{\Theta}_{g} \boldsymbol{\Theta}_{p} \boldsymbol{\Theta}_{g}$.

Proposition 5.54. The functor from Lemma 5.53 descends to a functor $\mathrm{T}_{e} \rightarrow \mathscr{E} \mathrm{nd}\left(\nabla_{e}\right)$. Moreover, decategorification gives the transitive $\mathbb{N}_{[\mathrm{v}]}^{0}$-representation $\mathrm{M}_{\mathbf{A}_{e}}$ of $\mathrm{T}_{e}$. 
Proof. Recall that the $\mathbb{N}_{[\mathrm{v}]}^{0}$-representation $\mathrm{M}_{\mathbf{A}_{e}}$ of $\mathrm{T}_{e}$ from Definition 5.27 satisfies

$$
\mathrm{M}_{\boldsymbol{\Gamma}}^{\text {tot }}=\mathrm{M}_{\boldsymbol{\Gamma}}\left(\theta_{g}\right)+\mathrm{M}_{\boldsymbol{\Gamma}}\left(\theta_{o}\right)+\mathrm{M}_{\boldsymbol{\Gamma}}\left(\theta_{p}\right)=[2]_{\mathrm{v}}\left([3]_{\mathrm{v}} \mathrm{Id}+A(\boldsymbol{\Gamma})\right) .
$$

Up to natural isomorphism, the same holds for the functors $\boldsymbol{\Theta}_{g}, \boldsymbol{\Theta}_{o}$ and $\boldsymbol{\Theta}_{p}$ when they are applied to $\mathrm{P}_{\mathbf{x}_{m, n},}, \mathrm{P}_{\mathrm{y}_{m, n}}$ and $\mathrm{P}_{\mathbf{z}_{m, n}}$, by Lemma 5.50. The proof uses exactly the same sort of arguments as the proof of Lemma 5.53. We therefore omit further details. The statement then follows from Corollary 5.36.

Remark 5.55. The quiver algebra defined in [Gra17] is (in the case of $\mathbf{A}_{e}$ graphs) a subalgebra of $\nabla_{e}$. We do not know any further connection between these two algebras.

In fact, up to certain scalars, the defining relations of the quiver algebra in Definition 5.48 are the ones of the quiver algebra underlying the cell 2-representations of $\mathscr{T}_{e}$. Those scalars can be computed for small values of $e$, but we have not been able to compute them for general $e$, unfortunately. However, even without the correct scalars, we thought that the trihedral zig-zag algebra, and its connection to [Gra17], was too nice to exclude it from this paper.

One also wonders whether any of the constructions involving the zig-zag algebras in [HK01] have an analogue for the trihedral zig-zag algebras.

\section{D. Generalizing dihedral 2-representation theory.}

The dihedral story 5.56. Yet another analogy to the dihedral case $\mathrm{D}_{e}$ is provided by CP 5.34 and Proposition 5.35: One can define transitive $\mathbb{N}_{[\mathrm{v}]}^{0}$-representations of $\mathrm{D}_{\infty}$ analogously to the $\mathbb{N}_{[\mathrm{v}]}^{0}$-representations $\mathrm{M}_{\boldsymbol{\Gamma}}$, where in the dihedral case $\boldsymbol{\Gamma}$ is any connected, bicolored graph. These descend to the finite-dimensional $\mathrm{D}_{e}$ if and only if $\mathrm{U}_{e+1}(A(\boldsymbol{\Gamma}))=0$, where $\mathrm{U}_{e+1}\left({ }_{-}\right)$is the Chebyshev polynomial as in 'The dihedral story' 3.24. (This follows from [KMMZ16] and, a bit more directly, from [MT16].) In that case, the analog of CP 5.34 has a well-understood answer, namely $\boldsymbol{\Gamma}$ has to be of ADE Dynkin type.

The dihedral story 5.57. In the dihedral case, the classification of simple transitive 2representation is an ADE-type classification (assuming gradeability), cf. [KMMZ16] and [MT16]. This follows from the classification of graphs recalled in 'The dihedral story' 5.56 and the associated 2-representations, which, in analogy to Section $5 \mathrm{~B}$, can be constructed using algebra 1-morphisms in the $\mathfrak{s l}_{2}$ analog of $\mathcal{Q}_{e}$ (cf. [MMMT16, Section 7]).

The dihedral story 5.58. The quiver underlying the cell 2-representations in the dihedral case is the zig-zag algebra from [HK01], which could be presented as in our setup, although this is never done in the literature, using two loops $\alpha_{\mathrm{x}}, \alpha_{\mathrm{y}}$ at each vertex, subject to the relations of the cohomology ring of the variety of full flags in $\mathbb{C}^{2}$, i.e. $\alpha_{\mathrm{x}} \alpha_{\mathrm{y}}=\alpha_{\mathrm{y}} \alpha_{\mathrm{x}}=0$, $\alpha_{\mathrm{x}}+\alpha_{\mathrm{y}}=0$. (To make the connection with [HK01], note that this cohomology ring is isomorphic to $\mathbb{C}[X] /\left(X^{2}\right)$.) The same holds for all other simple transitive 2-representations (in the dihedral case) with the zig-zag algebra for the corresponding graph, see [MT16]. Thus, Section $5 \mathrm{C}$ can be seen as the trihedral version of this. We think it would be interesting to work out the trihedral quiver algebras for other simple transitive 2-representations of $\mathfrak{T}_{e}$.

\section{Appendix: Generalized ADE Dynkin Diagrams}

In this appendix we have listed certain solutions to CP 5.34. Following [Zub98], we call these the generalized ADE Dynkin diagrams. The graphs below depend on the level $e$, which is the same as e.g. in Section 2 and is indicated as a subscript.

AppA. The list. The following are the generalized ADE Dynkin diagrams. There are three infinite families, displayed in Figures App-1, App-2 and App-3, and a finite number of exceptions, displayed in Figure App-4. 


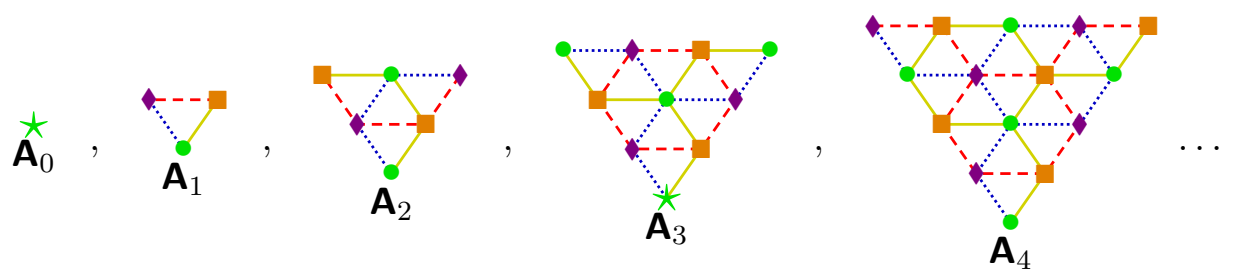

Figure ApP-1. The infinite family of (generalized) type A, indexed by $e \in \mathbb{N}^{0}$. The graph of type $\mathbf{A}_{e}$ can be obtained by cutting off the $\mathfrak{s l}_{3}$-weight lattice at level $e+1$, as in $(2-1)$.

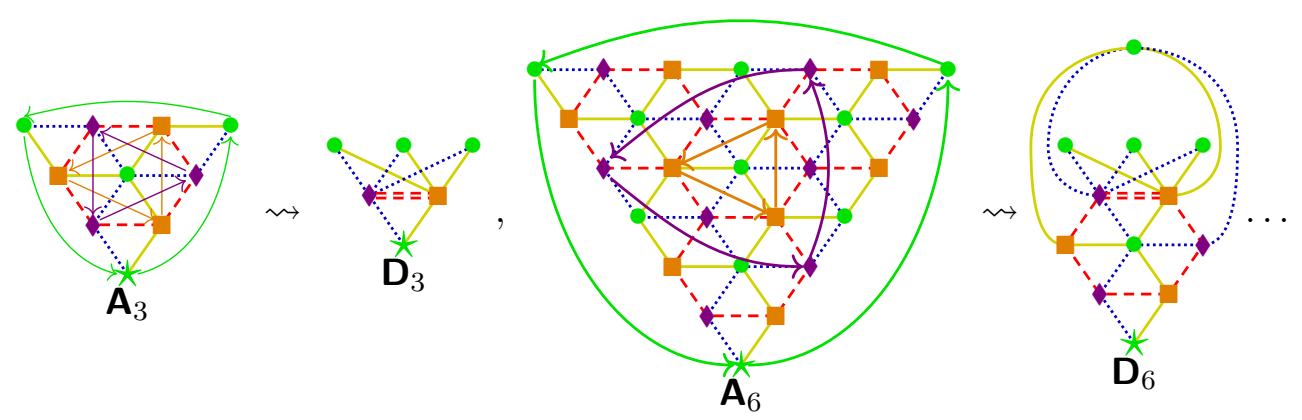

Figure ApP-2. The infinite family of (generalized) type D, indexed by $e \equiv 0 \bmod 3$ and $e \neq 0$. The graph of type $\mathbf{D}_{e}$ comes from the $\mathbb{Z} / 3 \mathbb{Z}$-symmetry of the graph of type $\mathbf{A}_{e}$. (Note the double edges.) By convention, $\mathbf{A}_{0}=\mathbf{D}_{0}$.

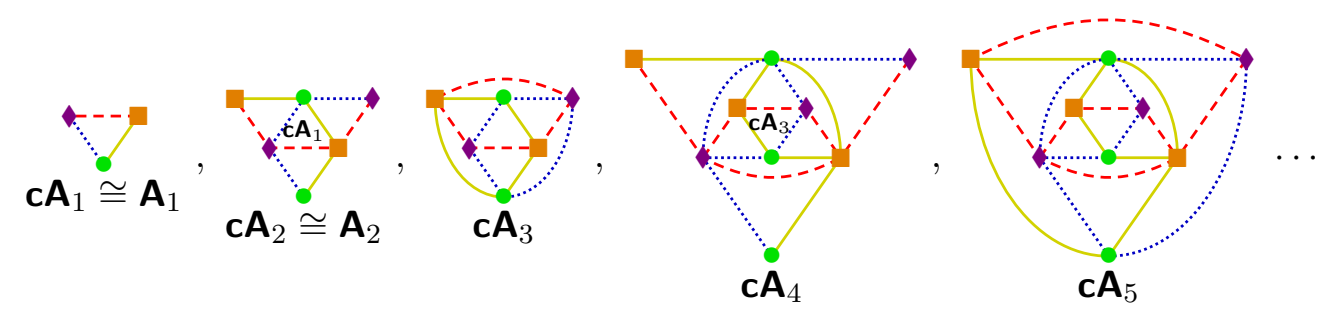

Figure App-3. The infinite family of conjugate type $A$, indexed by $e \in \mathbb{N}^{0}$. The graph of type $\mathbf{c} \mathbf{A}_{e}$ comes from an iterative procedure on the graph of type $\mathbf{A}_{e}$. (Note the double edges.) By convention, $\mathbf{A}_{0}=\mathbf{c} \mathbf{A}_{0}$.

All the above graphs exist for color variations as well. Note further that we have also indicated a starting vertex $\star$ in case such a choice is essential, i.e. in case different tricolorings give non-isomorphic tricolored graphs.

We point out that the above list was obtained from [Ocn02, Section 4] by excluding the ones that are not tricolorable.

AppB. The spectra. The spectra of the graphs from Section AppA are known, cf. [EP10, Section 2]. Let us sketch how they look like. To this end, recall vanishing set $\mathrm{V}_{e}$ of level $e$ and the discoid $\mathrm{d}_{3}$ from Section $2 \mathrm{~B}$.

'Section AppB.ClaimA'. $z \in S_{\mathbf{A}_{e}^{\mathrm{x}}}$ if and only if $(z, \bar{z}) \in \mathrm{V}_{e}$. 

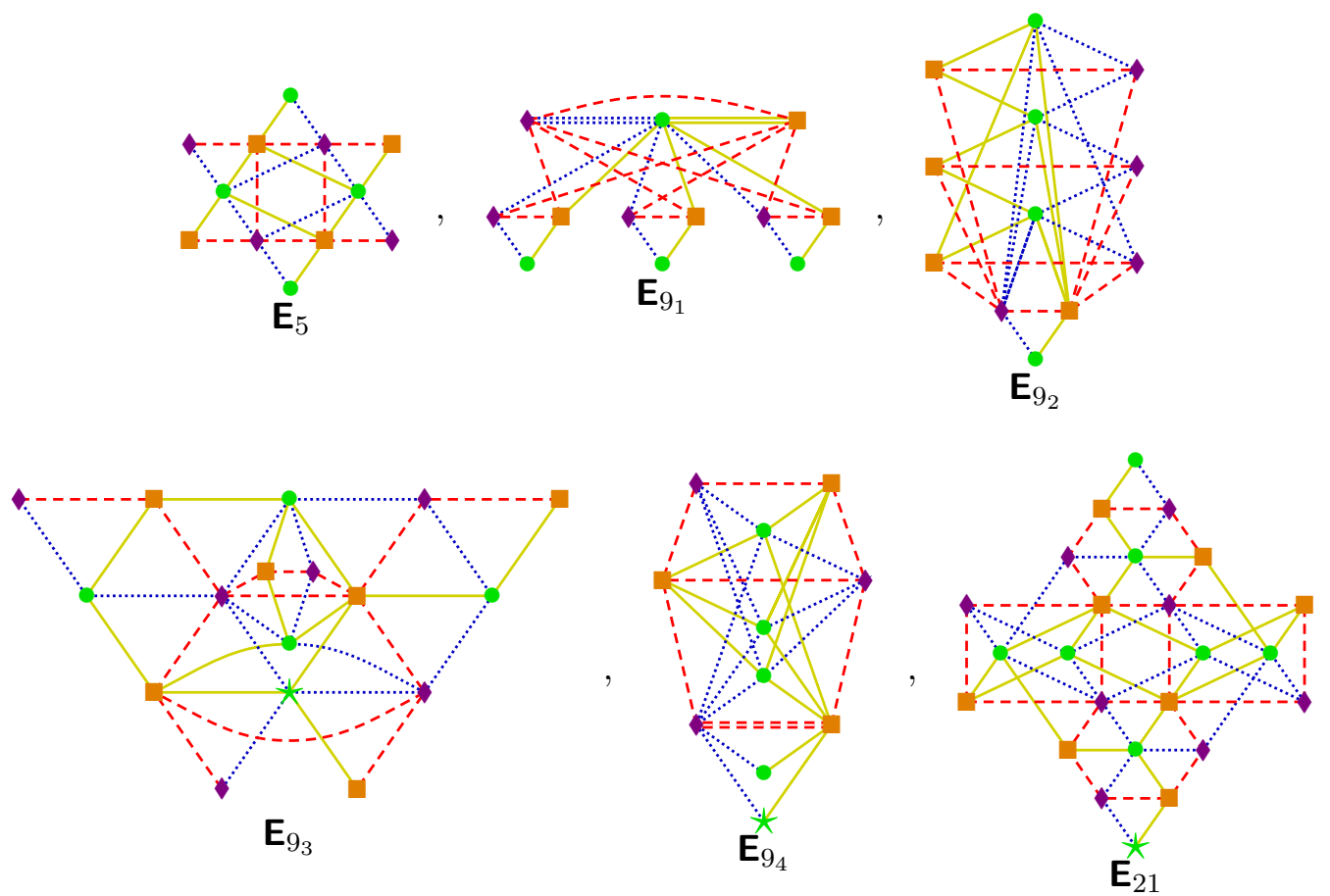

FIgURE ApP-4. The finite exceptional family of (generalized) type E, indexed as indicated and denoted by $\mathbf{E}_{e}$. (Note that there are four of level $e=9$.)

Proof(Sketch) of 'Section AppB.ClaimA'. Observe that $\mathbf{A}_{e}^{\mathbf{X}}$ and $\mathbf{A}_{e}^{\mathbf{Y}}$ are the graphs encoding the action of $\left[\mathrm{X} \otimes_{-}\right]$, respectively of $\left[\mathrm{Y} \otimes_{-}\right]$, on $\left[\mathcal{Q}_{e}\right]_{\oplus}^{\mathbb{C}}$, and the claim follows.

'Section AppB.ClaimDE'. We have, without counting multiplicities of the zero eigenvalue, $S_{\Gamma^{\mathrm{x}}} \subset S_{\mathbf{A}_{e}^{\mathrm{x}}}$ for any $\boldsymbol{\Gamma}$ as in Section AppA.

Proof(Sketch) of 'Section AppB.ClaimDE'. For graphs of type $\mathbf{D}_{e}$ this holds by virtue of their construction, using the $\mathbb{Z} / 3 \mathbb{Z}$-symmetry of the $\mathbf{A}_{e}$ graphs. In fact, one can get the eigenvalues of $A\left(\mathbf{D}_{e}\right)$ from the ones of $A\left(\mathbf{A}_{e}\right)$ by deleting two out of every three eigenvalues and adding two additional eigenvalues 0 , e.g. for $e=3$ :

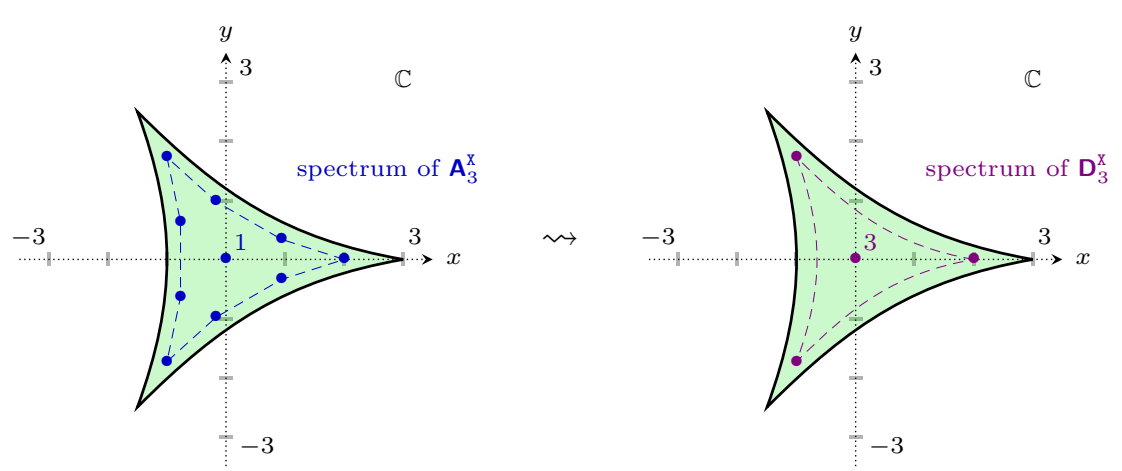


The case of $\mathbf{c} \mathbf{A}_{e}$ can be shown similarly (precisely which eigenvalues of $S_{\mathbf{A}_{e}}$ also belong to $S_{\mathbf{c A}_{e}}$ depends on $e$ mod 3), with a prototypical example given by:

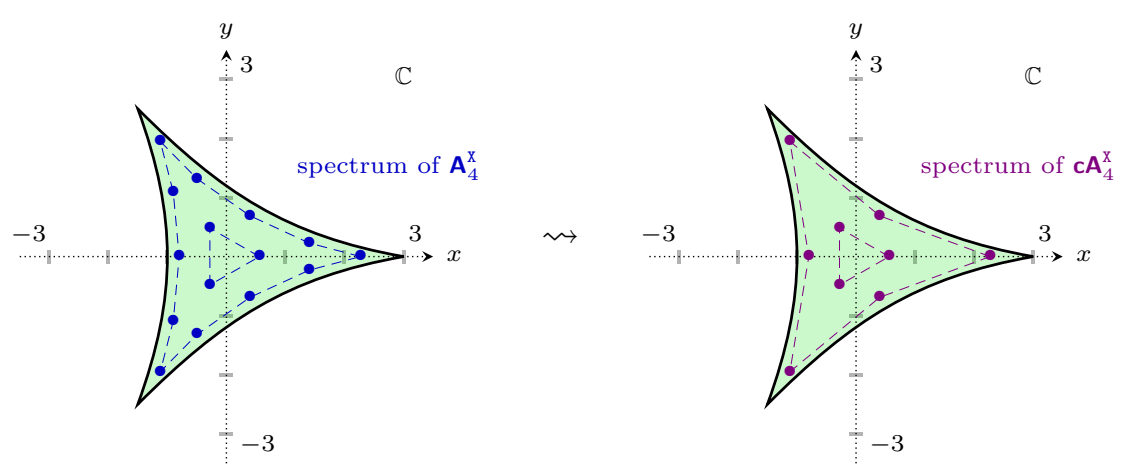

For the exceptional type $\mathrm{E}$ graphs the claim can be checked case-by-case.

In particular, the spectra of the generalized ADE Dynkin diagrams are all inside $\mathrm{d}_{3}$.

Example App.1. The spectra of $\mathbf{A}_{1}^{\mathrm{X}}, \mathbf{A}_{2}^{\mathrm{X}}$ and $\mathbf{A}_{3}^{\mathrm{X}}$ are given in Example 5.23. (Again, these should be compared to Example 2.12.) Additionally we have

$$
S_{\mathbf{D}_{3}^{\mathrm{x}}}=S_{\mathbf{c A} \mathbf{x}}=\left\{\text { roots of } X^{3}(X-2)\left(X^{2}+2 X+4\right)\right\}
$$

Forgetting the multiplicity of zero, we get the inclusion of the corresponding spectra.

Example App.2. The graphs $\mathbf{D}_{3}^{\mathrm{X}}$ and $\mathbf{c A}_{3}^{\mathrm{X}}$ have the same spectrum, cf. Example App.1. However, they are not isomorphic as graphs, e.g. $\mathbf{D}_{3}$ has a double-edge and $\mathbf{c A}_{3}^{\mathbf{X}}$ does not. Both observations are true in general for $\mathbf{D}_{e}$ and $\mathbf{c} \mathbf{A}_{e}$.

AppC. Zuber's classification problem and CP 5.34. Zuber (based on joint work with Di Francesco [DFZ90] and Petkova [PZ96]) introduced the notion of a generalized ADE Dynkin diagram. These graphs appear in various disguises in the literature, e.g. in conformal field theories, integrable lattice models, topological field theories for 3-manifold invariants and subfactor theory.

Zuber wrote down a list of six axioms which these graphs should satisfy, see [Zub98, Section 1.2], and asked for the classification of such graphs. In [Ocn02], Ocneanu argued that Zuber's classification problem is related to the classification problem of the so-called quantum subgroups of $\mathrm{SU}(N)$. He also proposed a list of graphs which should solve Zuber's classification problem. The ones that are tricolorable are the graphs that we reproduced in Section AppA. However, we already saw in Theorem 5.38 that we get solutions which are not on Ocneanu's list, so we do not know whether CP 5.34 and Zuber's classification problem are the same or not, or even how they are related.

\section{REFERENCES}

[AP95] H.H. Andersen and J. Paradowski. Fusion categories arising from semisimple Lie algebras. Comm. Math. Phys., 169(3):563-588, 1995.

[APW91] H.H. Andersen, P. Polo, and K.X. Wen. Representations of quantum algebras. Invent. Math., 104(1):1-59, 1991. doi:10.1007/BF01245066.

[AT17] H.H. Andersen and D. Tubbenhauer. Diagram categories for $\mathbf{U}_{q}$-tilting modules at roots of unity. Transform. Groups, 22(1):29-89, 2017. URL: http://arxiv.org/abs/1409.2799, doi: 10.1007/s00031-016-9363-z.

[BH12] A.E. Brouwer and W.H. Haemers. Spectra of graphs. Universitext. Springer, New York, 2012. doi:10.1007/978-1-4614-1939-6.

[BK01] B. Bakalov and A. Kirillov, Jr. Lectures on tensor categories and modular functors, volume 21 of University Lecture Series. American Mathematical Society, Providence, RI, 2001. 
[BZ08] A. Berenstein and S. Zwicknagl. Braided symmetric and exterior algebras. Trans. Amer. Math. Soc., 360(7):3429-3472, 2008. URL: https://arxiv.org/abs/math/0504155, doi:10. 1090/S0002-9947-08-04373-0.

[CM16] A. Chan and W. Mazorchuk. Diagrams and discrete extensions for finitary 2-representations. 2016. To appear in Math. Proc. Camb. Phil. Soc. URL: https://arxiv.org/abs/1601.00080.

[DFZ90] P. Di Francesco and J.-B. Zuber. SU $(N)$ lattice integrable models associated with graphs. Nuclear Phys. B, 338(3):602-646, 1990. doi:10.1016/0550-3213(90)90645-T.

[EGNO15] P. Etingof, S. Gelaki, D. Nikshych, and V. Ostrik. Tensor categories, volume 205 of Mathematical Surveys and Monographs. American Mathematical Society, Providence, RI, 2015. doi:10.1090/ surv/205.

[EK95] P. Etingof and M. Khovanov. Representations of tensor categories and Dynkin diagrams. Internat. Math. Res. Notices, (5):235-247, 1995. URL: https://arxiv.org/abs/hep-th/9408078, doi: 10.1155/S1073792895000183.

[Eli15] B. Elias. Light ladders and clasp conjectures. 2015. URL: https://arxiv.org/abs/1510.06840.

[Eli16] B. Elias. The two-color Soergel calculus. Compos. Math., 152(2):327-398, 2016. URL: http: //arxiv.org/abs/1308.6611, doi:10.1112/S0010437X15007587.

[Eli17] B. Elias. Quantum Satake in type A. Part I. J. Comb. Algebra, 1(1):63-125, 2017. URL: https: //arxiv.org/abs/1403.5570, doi:10.4171/JCA/1-1-4.

[EP10] D.E. Evans and M. Pugh. Spectral measures and generating series for nimrep graphs in subfactor theory. Comm. Math. Phys., 295(2):363-413, 2010. URL: https://arxiv.org/abs/0906.4314, doi:10.1007/s00220-009-0902-5.

[ESW17] B. Elias, N. Snyder, and G. Williamson. On cubes of Frobenius extensions. In Representation theory - current trends and perspectives, EMS Ser. Congr. Rep., pages 171-186. Eur. Math. Soc., Zürich, 2017. URL: https://arxiv.org/abs/1308.5994.

[EW16] B. Elias and G. Williamson. Soergel calculus. Represent. Theory, 20:295-374, 2016. URL: https: //arxiv.org/abs/1309.0865, doi:10.1090/ert/481.

[Ful89] W. Fulton. Algebraic curves. Advanced Book Classics. Addison-Wesley Publishing Company, Advanced Book Program, Redwood City, CA, 1989. An introduction to algebraic geometry, Notes written with the collaboration of Richard Weiss, Reprint of 1969 original.

[Gan94] T. Gannon. The classification of affine SU(3) modular invariant partition functions. Comm. Math. Phys., 161(2):233-263, 1994. URL: https://arxiv.org/abs/hep-th/9212060.

[Gra17] J. Grant. Higher zig-zag algebras. 2017. URL: https://arxiv.org/abs/1711.00794.

[HK01] R.S. Huerfano and M. Khovanov. A category for the adjoint representation. J. Algebra, 246(2):514542, 2001. URL: https://arxiv.org/abs/math/0002060, doi:10.1006/jabr.2001.8962.

[Jan96] J.C. Jantzen. Lectures on quantum groups, volume 6 of Graduate Studies in Mathematics. American Mathematical Society, Providence, RI, 1996.

[Kim07] D. Kim. Jones-Wenzl idempotents for rank 2 simple Lie algebras. Osaka J. Math., 44(3):691-722, 2007. URL: https://arxiv.org/abs/math/0602504.

[KJO02] A. Kirillov Jr. and V. Ostrik. On a $q$-analogue of the McKay correspondence and the ADE classification of $\mathfrak{s l}_{2}$ conformal field theories. Adv. Math., 171(2):183-227, 2002. URL: http: //arxiv.org/abs/math/0101219, doi:10.1006/aima.2002.2072.

[KL79] D. Kazhdan and G. Lusztig. Representations of Coxeter groups and Hecke algebras. Invent. Math., 53(2):165-184, 1979. doi:10.1007/BF01390031.

[KM16] T. Kildetoft and V. Mazorchuk. Special modules over positively based algebras. Doc. Math., 21:1171-1192, 2016. URL: https://arxiv.org/abs/1601.06975.

[KMMZ16] T. Kildetoft, M. Mackaay, V. Mazorchuk, and J. Zimmermann. Simple transitive 2-representations of small quotients of Soergel bimodules. 2016. To appear in Trans. Amer. Math. Soc. URL: http://arxiv.org/abs/1605.01373.

[KMS09] M. Khovanov, V. Mazorchuk, and C. Stroppel. A brief review of abelian categorifications. Theory Appl. Categ., 22:No. 19, 479-508, 2009. URL: https://arxiv.org/abs/math/0702746.

[Koo74] T.H. Koornwinder. Orthogonal polynomials in two variables which are eigenfunctions of two algebraically independent partial differential operators. I-IV. Nederl. Akad. Wetensch. Proc. Ser. A 77=Indag. Math., 36:48-66,357-381, 1974.

[KS02] M. Khovanov and P. Seidel. Quivers, Floer cohomology, and braid group actions. J. Amer. Math. Soc., 15(1):203-271, 2002. URL: http://arxiv.org/abs/math/0006056, doi:10.1090/ S0894-0347-01-00374-5.

[Kup96] G. Kuperberg. Spiders for rank 2 Lie algebras. Comm. Math. Phys., 180(1):109-151, 1996. URL: http://arxiv.org/abs/q-alg/9712003.

[Lau10] A.D. Lauda. A categorification of quantum sl(2). Adv. Math., 225(6):3327-3424, 2010. URL: https://arxiv.org/abs/0803.3652, doi:10.1016/j.aim.2010.06.003. 
[Lei98] T. Leinster. Basic bicategories. 1998. eprint. URL: https://arxiv.org/abs/math/9810017.

[Lus83] G. Lusztig. Some examples of square integrable representations of semisimple $p$-adic groups. Trans. Amer. Math. Soc., 277(2):623-653, 1983. doi:10.2307/1999228.

[Lus87] G. Lusztig. Leading coefficients of character values of Hecke algebras. In The Arcata Conference on Representations of Finite Groups (Arcata, Calif., 1986), volume 47 of Proc. Sympos. Pure Math., pages 235-262. Amer. Math. Soc., Providence, RI, 1987.

[Lus90] G. Lusztig. Finite-dimensional Hopf algebras arising from quantized universal enveloping algebra. J. Amer. Math. Soc., 3(1):257-296, 1990. doi:10.2307/1990988.

[ML98] S. Mac Lane. Categories for the working mathematician, volume 5 of Graduate Texts in Mathematics. Springer-Verlag, New York, second edition, 1998.

[MM11] V. Mazorchuk and V. Miemietz. Cell 2-representations of finitary 2-categories. Compos. Math., 147(5):1519-1545, 2011. URL: https://arxiv.org/abs/1011.3322, doi:10.1112/ S0010437X11005586.

[MM16a] V. Mazorchuk and V. Miemietz. Endomorphisms of cell 2-representations. Int. Math. Res. Not. IMRN, (24):7471-7498, 2016. URL: https://arxiv.org/abs/1207.6236, doi:10.1093/imrn/ rnw025.

[MM16b] V. Mazorchuk and V. Miemietz. Transitive 2-representations of finitary 2-categories. Trans. Amer. Math. Soc., 368(11):7623-7644, 2016. URL: https://arxiv.org/abs/1404.7589, doi: $10.1090 / \operatorname{tran} / 6583$.

[MMMT16] M. Mackaay, V. Mazorchuk, V. Miemietz, and D. Tubbenhauer. Simple transitive 2-representations via (co)algebra 1-morphisms. 2016. To appear in Indiana Univ. Math. J. URL: https://arxiv . org/abs/1612.06325.

[MMMZ18] M. Mackaay, V. Mazorchuk, V. Miemietz, and X. Zhang. Analogues of centralizer subalgebras for fiat 2-categories and their 2-representations. 2018. URL: https://arxiv .org/abs/1802.02078.

[MT16] M. Mackaay and D. Tubbenhauer. Two-color Soergel calculus and simple transitive 2representations. 2016. To appear in Canad. J. Math. URL: https://arxiv.org/abs/1609.00962, doi:10.4153/CJM-2017-061-2.

[Ocn02] A. Ocneanu. The classification of subgroups of quantum $\mathrm{SU}(N)$. In Quantum symmetries in theoretical physics and mathematics (Bariloche, 2000), volume 294 of Contemp. Math., pages 133-159. Amer. Math. Soc., Providence, RI, 2002. doi:10.1090/conm/294/04972.

[Ost03] V. Ostrik. Module categories, weak Hopf algebras and modular invariants. Transform. Groups, 8(2):177-206, 2003. URL: https://arxiv.org/abs/math/0111139, doi:10.1007/ s00031-003-0515-6.

[PZ96] V. B. Petkova and J.-B. Zuber. From CFT to graphs. Nuclear Phys. B, 463(1):161-193, 1996. URL: https://arxiv.org/abs/hep-th/9510175, doi:10.1016/0550-3213(95)00670-2.

[QS15] H. Queffelec and A. Sartori. Mixed quantum skew Howe duality and link invariants of type A. 2015. URL: https://arxiv.org/abs/1504.01225.

[Rou08] R. Rouquier. 2-Kac-Moody algebras. 2008. URL: https://arxiv.org/abs/0812.5023.

[RT16] D.E.V. Rose and D. Tubbenhauer. Symmetric webs, Jones-Wenzl recursions, and $q$-Howe duality. Int. Math. Res. Not. IMRN, (17):5249-5290, 2016. URL: http://arxiv.org/abs/1501.00915, doi:10.1093/imrn/rnv302.

[RW18] L.H. Robert and E. Wagner. Symmetric Khovanov-Rozansky link homologies. 2018. URL: https://arxiv.org/abs/1801.02244.

[Saw06] S.F. Sawin. Quantum groups at roots of unity and modularity. J. Knot Theory Ramifications, 15(10):1245-1277, 2006. URL: https://arxiv.org/abs/math/0308281, doi:10.1142/ S0218216506005160.

[Sch17] A. Schopieray. Classification of $\mathfrak{s l}_{3}$ relations in the Witt group of nondegenerate braided fusion categories. Comm. Math. Phys., 353(3):1103-1127, 2017. URL: https://arxiv.org/abs/1608. 03155.

[Smi70] J.H. Smith. Some properties of the spectrum of a graph. In Combinatorial Structures and their Applications (Proc. Calgary Internat. Conf., Calgary, Alta., 1969), pages 403-406. Gordon and Breach, New York, 1970.

[Soe92] W. Soergel. The combinatorics of Harish-Chandra bimodules. J. Reine Angew. Math., 429:49-74, 1992. doi:10.1515/crll.1992.429.49.

[Soe97] W. Soergel. Kazhdan-Lusztig polynomials and a combinatoric[s] for tilting modules. Represent. Theory, 1:83-114, 1997. doi:10.1090/S1088-4165-97-00021-6.

[TVW17] D. Tubbenhauer, P. Vaz, and P. Wedrich. Super $q$-Howe duality and web categories. Algebr. Geom. Topol., 17(6):3703-3749, 2017. URL: https://arxiv.org/abs/1504.05069. 
[Zim17] J. Zimmermann. Simple transitive 2-representations of Soergel bimodules in type $B_{2}$. J. Pure Appl. Algebra, 221(3):666-690, 2017. URL: https://arxiv.org/abs/1509.01441, doi:10.1016/ j.jpaa.2016.07.011.

[Zub98] J.-B. Zuber. Generalized Dynkin diagrams and root systems and their folding. In Topological field theory, primitive forms and related topics (Kyoto, 1996), volume 160 of Progr. Math., pages 453-493. Birkhäuser Boston, Boston, MA, 1998. URL: https://arxiv .org/abs/hep-th/9707046.

M.M.: Center for Mathematical Analysis, Geometry, and Dynamical Systems, Departamento de Matemática, Instituto Superior Técnico, 1049-001 Lisboa, Portugal \& Departamento de Matemática, FCT, Universidade do Algarve, Campus de Gambelas, 8005-139 Faro, Portugal E-mail address: mmackaay@ualg.pt

Vo.ma.: Department of Mathematics, Uppsala University, Box. 480, SE-75106, Uppsala, SWEDEN

E-mail address: mazor@math.uu.se

Va.Mi.: School of Mathematics, University of East Anglia, Norwich NR4 7TJ, United Kingdom E-mail address: v.miemietz@uea.ac.uk

D.T.: Institut für Mathematik, Universität Zürich, Winterthurerstrasse 190, Campus Irchel, OfFice Y27J32, CH-8057 Zürich, Switzerland, Www.DtubBenhauer.COM

E-mail address: daniel.tubbenhauer@math.uzh.ch 